\title{
A new decision-making approach for multiple criteria sorting with an imbalanced set of assignment examples
}

DOI:

10.1016/j.ejor.2017.07.043

\section{Document Version}

Accepted author manuscript

Link to publication record in Manchester Research Explorer

\section{Citation for published version (APA):}

Liu, J. P., Liao, X. W., Huang, W., \& Yang, J-B. (2017). A new decision-making approach for multiple criteria sorting with an imbalanced set of assignment examples. European Journal of Operational Research, 265(2), 598620. https://doi.org/10.1016/j.ejor.2017.07.043

\section{Published in:}

European Journal of Operational Research

\section{Citing this paper}

Please note that where the full-text provided on Manchester Research Explorer is the Author Accepted Manuscript or Proof version this may differ from the final Published version. If citing, it is advised that you check and use the publisher's definitive version.

\section{General rights}

Copyright and moral rights for the publications made accessible in the Research Explorer are retained by the authors and/or other copyright owners and it is a condition of accessing publications that users recognise and abide by the legal requirements associated with these rights.

\section{Takedown policy}

If you believe that this document breaches copyright please refer to the University of Manchester's Takedown Procedures [http://man.ac.uk/04Y6Bo] or contact uml.scholarlycommunications@manchester.ac.uk providing relevant details, so we can investigate your claim.

\section{OPEN ACCESS}




\section{Accepted Manuscript}

A new decision-making approach for multiple criteria sorting with an imbalanced set of assignment examples

Jiapeng Liu, Xiuwu Liao, Wei Huang, Jian-bo Yang

PII:

S0377-2217(17)30670-7

DOI:

10.1016/j.ejor.2017.07.043

Reference: $\quad$ EOR 14593

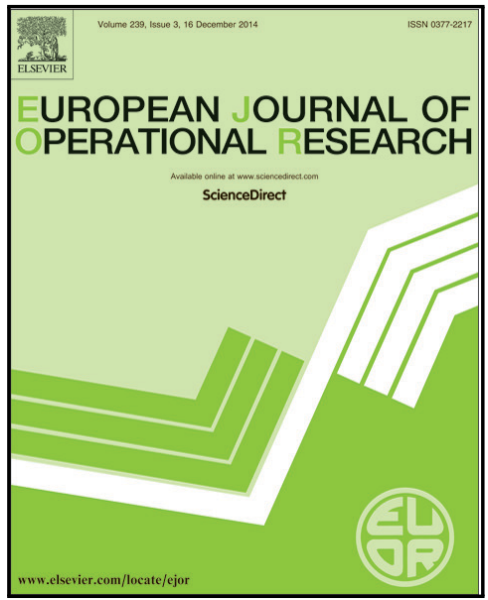

To appear in:

European Journal of Operational Research

Received date:

8 November 2016

Revised date:

11 July 2017

Accepted date:

13 July 2017

Please cite this article as: Jiapeng Liu, Xiuwu Liao, Wei Huang, Jian-bo Yang, A new decision-making approach for multiple criteria sorting with an imbalanced set of assignment examples, European Journal of Operational Research (2017), doi: 10.1016/j.ejor.2017.07.043

This is a PDF file of an unedited manuscript that has been accepted for publication. As a service to our customers we are providing this early version of the manuscript. The manuscript will undergo copyediting, typesetting, and review of the resulting proof before it is published in its final form. Please note that during the production process errors may be discovered which could affect the content, and all legal disclaimers that apply to the journal pertain. 


\section{Highlights}

- Multiple criteria sorting with imbalanced set of assignment examples is addressed.

- Clustering analysis and linear programming are used to identify active reference alternatives.

- A balancing algorithm is developed to balance active reference alternatives across categories.

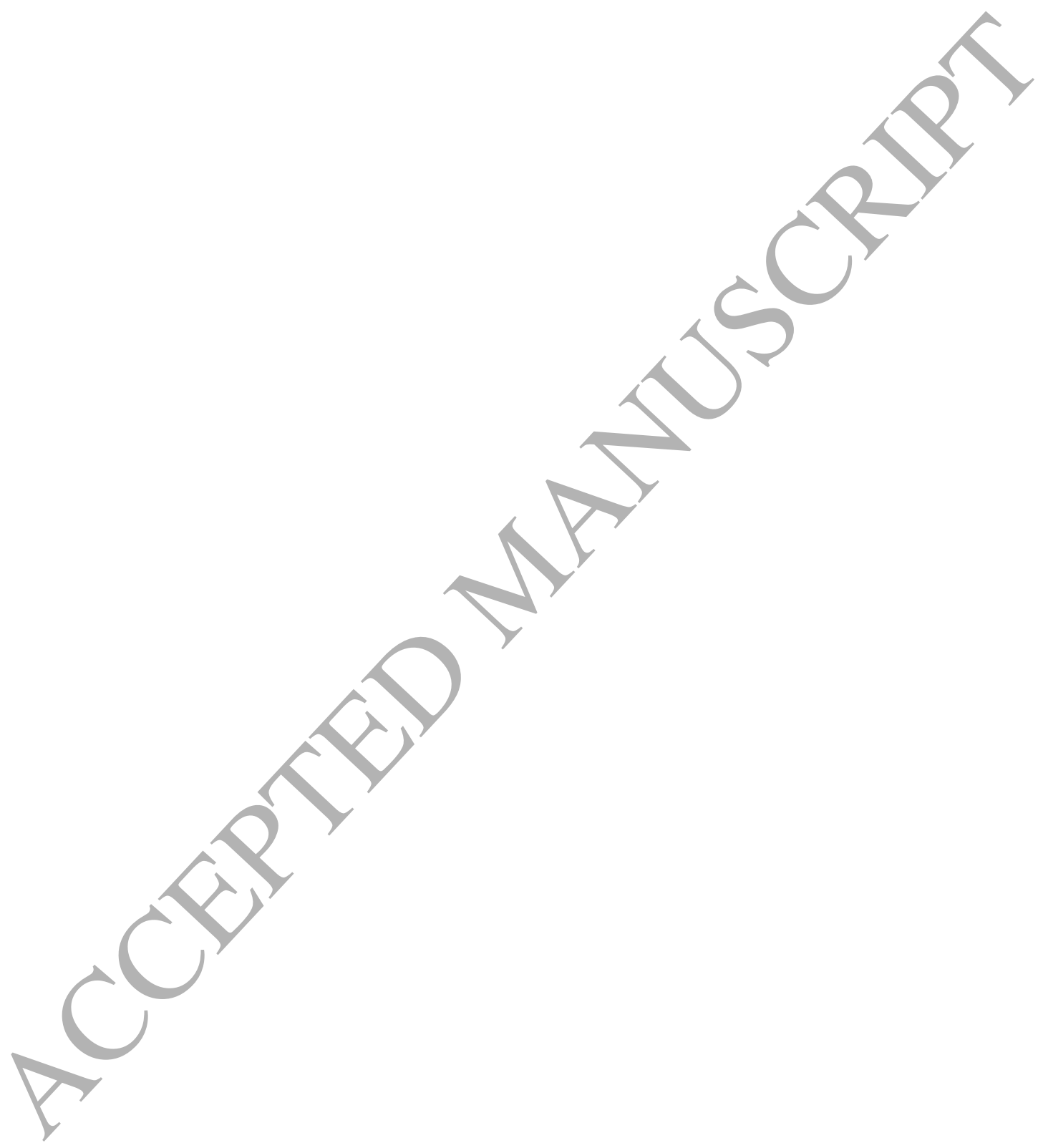




\title{
A new decision-making approach for multiple criteria sorting with an imbalanced set of assignment examples
}

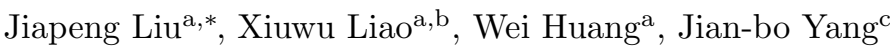 \\ ${ }^{a}$ School of Management, The Key Lab of the Ministry of Education for Process Control and Efficiency Engineering, Xi'an \\ Jiaotong University, Xi'an, 710049, Shaanxi, PR China \\ ${ }^{b}$ Collaborative Innovation Center of China Pilot Reform Exploration and Assessment - Hubei Sub-Center, Hubei University of \\ Economics, Wuhan, 430205, Hubei, PR China \\ ${ }^{c}$ Alliance Manchester Business School, The University of Manchester, Manchester M13 9SS, UK
}

\begin{abstract}
We propose a novel approach to address a multiple criteria sorting (MCS) problem, with an imbalanced set of assignment examples. The approach employs a piecewise-linear additive value function as the preference model and adopts the disaggregation-aggregation paradigm to infer a sorting model from provided assignment examples on a set of reference alternatives. We utilize a hierarchical clustering algorithm and several linear programming models to identify reference alternatives that are active to develop the sorting model, so that inactive ones are eliminated from the whole set of reference alternatives. Then, in order to construct a balanced set of assignment examples, a balancing algorithm is proposed to balance active reference alternatives across categories. Finally, the sorting model is obtained by minimizing the sum of violations between values of active reference alternatives and corresponding category thresholds. Furthermore, the performance of the proposed approach is investigated on a hypothetical problem and several real data sets. The experimental results show that our approach is efficient to address the MCS problem with an imbalanced set of assignment examples.
\end{abstract} Keywords: Multiple criteria analysis, Multiple criteria sorting, Imbalanced set of assignment examples, Clustering analysis

\section{Introduction}

In multiple criteria sorting (MCS, also called ordinal classification) problems, a decision maker (DM) needs to assign a finite set of alternatives to several predefined and preferentially ordered categories according to multiple evaluation criteria. Such a classification decision depends on the preference information provided by the DM, which could be either direct or indirect. The direct preference information refers to the specification of precise values of or constraints on parameters of a preference model, such as weights, trade-offs, category profiles, etc. On the contrast, the indirect preference information consists of assignment examples on a set of reference alternatives. Each assignment example specifies a desired assignment of a corresponding reference alternative to one or several contiguous categories [1]. Such reference alternatives could come from either past decisions, or a subset of alternatives under consideration, or a set of fictitious alternatives [2]. It is more

\footnotetext{
* Corresponding author

Email address: jiapengliu@mail.xjtu.edu.cn (Jiapeng Liu)
} 
user-friendly to ask the DM to provide indirect preference information than the direct one, because the former requires less cognitive effort from the DM at the stage of preference elicitation $[3,4,5]$.

Indirect preference information is used in the disaggregation-aggregation paradigm that aims at inferring compatible instances of a preference model to restore the assignment examples provided by the DM. MCS methods based on the indirect preference information and the disaggregation-aggregation paradigm have been implemented with four main families of preference models: a value function $[6,7,8,9$, 10], an outranking relation $[11,12,13,14,15,16]$, a distance model [17, 18], and a set of decision rules [19, 20, 21].

Generally, there could exist more than one set of parameters of a preference model compatible with the preference information given by the DM. Usually, from among these compatible sets of parameters, traditional MCS methods, such as the UTADIS method [6], select one specific set to work out an assignment recommendation. Such a choice is arbitrary because two different compatible sets could achieve different assignments for an alternative although they restore the preference information provided by the DM. To avoid considering only one specific set chosen from the whole family of compatible sets in an arbitrary way, the principle of Robust Ordinal Regression (ROR) [22] is proposed to take into account all the sets of parameters compatible with the preference information, in order to give a recommendation in terms of necessary and possible consequences of applying all the compatible preference models on the considered set of alternatives. Ref. [1] first applied the principle of ROR to MCS and proposed the UTADISGMS method that computes two sorting results for each alternative: the possible assignment and the necessary assignment. Then, the principle of ROR has been adapted to MCS in context of group decision-making [23, 24], outranking-based MCS [15], value-driven MCS [9], and MCS in case of a hierarchical structure of the family of evaluation criteria [25].

Although existing MCS methods have shown great success in many real-world applications [26, 27, 28, $29,30,31,32,33,34,35,36,37,38]$, the complexity of some practical problems makes them inadequate to achieve a satisfactory assignment recommendation. One noteworthy difficulty is the imbalanced distribution of alternatives among considered categories, which exists in a wide range of real-world applications. For example, in credit risk assessment, firms are classified into two classes by a bank loan officer: default and non-default, and the number of default firms is significantly less than that of non-default firms [39, 28, 40]; in ABC inventory classification, inventory items are assigned to three classes according to specific criteria, items of high value but small in number are termed as class A, items of low value but large in number are termed as class $\mathrm{C}$, and items that fall between these two classes are termed as class B [27]; in engineering management, activities carried out by a project team are assigned into classes of managerial practices, which include different control mechanisms for a project manager, and the class of activities which require most attention are usually small in number while the class of non-critical activities are large in quantity [34].

It is challenging to develop a sorting model from an imbalanced set of assignment examples. As pointed in [41], in the presence of severe distribution skews of assignment examples across categories, developing a model without considering this imbalance is likely to provide a imbalanced degree of accuracy, with excellent performances for larger categories, but poor performances for smaller ones. Existing MCS methods aim to infer a preference model to restore the provided assignment examples as much as possible (i.e., to obtain the 
minimum misclassification error). When dealing with an imbalanced set of assignment examples, these methods fail to properly represent the distributive characteristics of this set and would seek to obtain a high overall classification accuracy at the expense of deteriorating the accuracy for smaller categories to improve the one for larger categories. One needs to be aware that smaller categories usually consist of alternatives that are more important than those from larger ones in many real-world applications, and thus misclassification costs for alternatives in different categories are not always the same. For instance, the classification error regarding the assignment of a default firm to the category of non-default firms is much more costly than an error involving the assignment of a non-default firm to the default category. The former leads to capital cost (loss of the amount of credit granted to a firm), whereas the latter leads to opportunity cost (loss of profit that would result from granting a credit to a non-default firm) [6]. To cope with this imbalance, one can incorporate a cost matrix that describes the costs for misclassifying any particular assignment example into existing MCS methods to enable them to address an imbalanced set of assignment examples. However, it is often quite difficult to have reliable estimates for the cost of each type of misclassification in real-world MCS problems.

In this paper, we propose a new decision-making approach to deal with the MCS problem with an imbalanced set of assignment examples. The approach requires the DM to provide a set of assignment examples as the preference information and adopts the disaggregation-aggregation paradigm to develop a sorting model. We utilize a hierarchical clustering algorithm and several linear programming (LP) models to identify reference alternatives that are active to develop the sorting model, so that inactive ones are eliminated from the whole set of reference alternatives. Specifically, the hierarchical clustering algorithm is used to group reference alternatives of each category into clusters, and then the proposed LP models are solved to identify reference alternatives lying in preferential boundaries of each cluster, which are reference alternatives that are active to develop the sorting model. Then, in order to construct a balanced set of assignment examples, a balancing algorithm is proposed to balance active reference alternatives across categories. The algorithm uses an augmenting strategy to iteratively add artificial alternatives until the distribution of active reference alternatives across categories is adjusted to be balanced. Finally, the sorting model is obtained by minimizing the sum of violations between values of active reference alternatives and corresponding category thresholds.

The contributions of the proposed approach are three-fold as follows. First of all, our work is the first MCS approach to address an imbalanced set of assignment examples. To the best of our knowledge, although various MCS methods have been proposed in the literature, no work has devoted efforts to addressing the MCS problem with an imbalanced set of assignment examples. Our approach improves the classification performance in comparison with those MCS methods without considering the imbalance of assignment examples. The second contribution of this paper consists in utilizing the clustering analysis and LP models to identify active reference alternatives. In this way, we can get rid of those inactive reference alternatives in the following sorting model development. Thirdly, this paper proposes an augmenting mechanism to balance the distribution of active reference alternatives across categories. It can construct a balanced set of assignment examples.

The rest of the paper is organized as follows. Section 2 describes the proposed approach for addressing the MCS problem with an imbalanced set of assignment examples. Section 3 applies the proposed approach to 
solve a hypothetical MCS problem and reports the experimental results on real data sets. Section 4 ends with conclusions and discussion regarding future research.

\section{The proposed approach}

\subsection{Problem description}

We are considering a MCS problem which aims to assign a finite set of alternatives $A=\left\{a_{1}, a_{2}, \ldots, a_{m}\right\}$ to $l$ predefined and preferentially ordered categories $C=\left\{C_{1}, C_{2}, \ldots, C_{l}\right\}$ such that category $C_{s+1}$ is preferred to category $C_{s}, s=1,2, \ldots, l-1$. Such a classification decision is made based on the historical preference information provided a priori, which consists of a set of assignment examples on a finite set of reference alternatives $A^{R}=\left\{a_{1}^{*}, a_{2}^{*}, \ldots, a_{r}^{*}\right\}$. An assignment example specifies a reference alternative $a_{i}^{*} \in A^{R}$ and its assignment $C_{h\left(a_{i}^{*}\right)}, h\left(a_{i}^{*}\right)=1, \ldots, l$, which can be represented as $a_{i}^{*} \in C_{h\left(a_{i}^{*}\right)}$. Let $A_{s}^{R}$ denote the set of reference alternatives that are assigned to category $C_{s}$, i.e., $A_{s}^{R}=\left\{a_{i}^{*} \mid a_{i}^{*} \in A^{R}, a_{i}^{*} \in C_{s}\right\}$, and let $r_{s}$ denote the cardinality of $A_{s}^{R}$, i.e., $r_{s}=\left|A_{s}^{R}\right|, s=1,2, \ldots, l$. Thus, $A^{R}=A_{1}^{R} \cup \ldots \cup A_{l}^{R}$. Note that, for a MCS problem with an imbalanced set of assignment examples, there exist $s, s^{\prime}=1, \ldots, l$ such that $\left|A_{s}^{R}\right| \gg\left|A_{s^{\prime}}^{R}\right|$.

Each alternative $a \in A \cup A^{R}$ is evaluated on a family of criteria $G=\left\{g_{1}, g_{2}, \ldots, g_{n}\right\}$, where for all $g_{j} \in G$, $g_{j}(a)$ is the evaluation of alternative $a$ on criterion $g_{j}$. Without loss of generality, we assume that all criteria have an increasing direction of preference, i.e., the greater $g_{j}(a)$, the more preferred alternative $a$ on criterion $g_{j}$, for all $g_{j} \in G$ and $a \in A \cup A^{R}$. We assume that the evaluation scale on each criterion $g_{j} \in G$ is bounded and use $X_{j}=\left[\alpha_{j}, \beta_{j}\right]$ to denote the range of evaluations on $g_{j}$, where $\alpha_{j}$ and $\beta_{j}$ are the worst and best evaluations, respectively.

We assume that the criteria satisfy the mutual preference independence condition [42] and use an additive value function as the preference model:

$$
U(a)=\sum_{j=1}^{n} u_{j}\left(g_{j}(a)\right), a \in A \cup A^{R}
$$

where $U(a)$ is the global value of alternative $a$ and $u_{j}\left(g_{j}(a)\right), j=1, \ldots, n$, are the marginal value functions. For simplicity of notation, we write $u_{j}(a)$ instead of $u_{j}\left(g_{j}(a)\right)$.

In this paper, the marginal value function $u_{j}(a)$ is assumed to be a piecewise-linear function. The range $X_{j}=$ $\left[\alpha_{j}, \beta_{j}\right]$ is divided into $\gamma_{j} \geq 1$ equal sub-intervals $\left[x_{j}^{0}, x_{j}^{1}\right],\left[x_{j}^{1}, x_{j}^{2}\right], \ldots,\left[x_{j}^{\gamma_{j}-1}, x_{j}^{\gamma_{j}}\right]$, where $x_{j}^{k}=\alpha_{j}+\frac{k}{\gamma_{j}}\left(\beta_{j}-\alpha_{j}\right)$, $k=0,1, \ldots, \gamma_{j}$. The marginal value of alternative $a$ on criterion $g_{j}$ is obtained by linear interpolation:

$$
u_{j}(a)=u_{j}\left(x_{j}^{k_{j}}\right)+\frac{g_{j}(a)-x_{j}^{k_{j}}}{x_{j}^{k_{j}+1}-x_{j}^{k_{j}}}\left(u_{j}\left(x_{j}^{k_{j}+1}\right)-u_{j}\left(x_{j}^{k_{j}}\right)\right), \quad g_{j}(a) \in\left[x_{j}^{k_{j}}, x_{j}^{k_{j}+1}\right] .
$$

According to (2), the piecewise-linear value function is completely defined by marginal values at the characteristic points, i.e., $u_{j}\left(x_{j}^{0}\right)=u_{j}\left(\alpha_{j}\right), u_{j}\left(x_{j}^{1}\right), \ldots, u_{j}\left(x_{j}^{\gamma_{j}}\right)=u_{j}\left(\beta_{j}\right)[43]$. Note that we do not use general monotonic marginal value functions that consider all criteria values as characteristic points as in some formulations of the robust ordinal regression methodology $[22,1]$. Such a way could lead to the over-fitting of the developed sorting model to reference alternatives, and thus may deteriorate its generalization ability to non-reference 
alternatives. The assumption of piecewise-linear value function is appropriate for wide application because any nonlinear value function can be approximated given a sufficient number of characteristic points.

In particular, new variable $\Delta u_{j}^{t}$ is introduced to represent the difference between marginal values of two consecutive break-points $x_{j}^{t+1}$ and $x_{j}^{t}$ :

$$
\Delta u_{j}^{t}=u_{j}\left(x_{j}^{t+1}\right)-u_{j}\left(x_{j}^{t}\right), \quad t=0, \ldots, \gamma_{j}-1
$$

Thus, the marginal value $u_{j}(a)$ can be expressed as:

$$
u_{j}(a)=\sum_{t=0}^{k_{j}-1} \Delta u_{j}^{t}+\frac{g_{j}(a)-x_{j}^{k_{j}}}{x_{j}^{k_{j}+1}-x_{j}^{k_{j}}} \Delta u_{j}^{k_{j}}, \quad g_{j}(a) \in\left[x_{j}^{k_{j}}, x_{j}^{k_{j}+1}\right\}
$$

Definition 1. The characteristic vector $\mathbf{V}(a)$ of alternative $a \in A \cup A^{R}$ is defined as a column vector whose entries are the coefficients of variables $\Delta u_{j}^{t}$ in marginal values $u_{j}(a)$ on each criterion $g_{j}, j=1, \ldots, n$ :

$$
\mathbf{V}(a)=(\underbrace{\underbrace{1, \ldots, 1}_{k_{1}}, \frac{g_{1}(a)-x_{1}^{k_{1}}}{x_{1}^{k_{1}+1}-x_{1}^{k_{1}}}, \underbrace{0, \ldots, 0}_{\gamma_{1}-k_{1}-1}}_{\text {criterion } g_{1}}, \ldots, \underbrace{\underbrace{1, \ldots, 1}_{k_{j}}, \frac{g_{j}(a)-x_{j}^{k_{j}}}{x_{j}^{k_{j}+1}-x_{j}^{k_{j}}}, \underbrace{0, \ldots, 0}_{\gamma_{j}-k_{j}-1}}_{\text {criterion } g_{j}}, \ldots, \underbrace{\underbrace{1, \ldots, 1}_{k_{n}}, \frac{g_{n}(a)-x_{n}^{k_{n}}}{x_{n}^{k_{n}+1}-x_{n}^{k_{n}}}, \underbrace{0, \ldots, 0}_{\gamma_{n}-k_{n}-1}}_{\text {criterion } g_{n}})^{\mathrm{T}} .
$$

For alternative $a \in A \cup A^{R}$, with the definition/of characteristic vector $\mathbf{V}(a)$, the global value $U(a)$ can be formulated as

$$
U(a)=\mathbf{u}^{\mathrm{T}} \mathbf{V}(a)
$$

where $\mathbf{u}=(\underbrace{\Delta u_{1}^{0}, \ldots, \Delta u_{1}^{\gamma_{1}-1}}_{\text {criterion } g_{1}}, \ldots, \underbrace{\Delta u_{j}^{0}, \ldots, \Delta u_{j}^{\gamma_{j}-1}}_{\text {criterion } g_{j}}, \ldots, \underbrace{\Delta u_{n}^{0}, \ldots, \Delta u_{n}^{\gamma_{n}-1}}_{\text {criterion } g_{n}})^{\mathrm{T}}$ is a column vector.

To normalize the value function so that $U(a) \in[0,1], a \in A \cup A^{R}$, we set:

$$
\left.\begin{array}{l}
\mathbf{e}^{\mathrm{T}} \mathbf{u}=1 \\
\mathbf{u} \geq \mathbf{0},
\end{array}\right\}
$$

where $\mathbf{e} \in \mathbb{R}^{\gamma}$ is a column vector whose entries are all equal to $1, \gamma=\sum_{j=1}^{n} \gamma_{j}$.

In this paper, we consider the threshold-based sorting procedure [1], where a vector of category thresholds $\mathbf{b}=\left(b_{0}, b_{1}, \ldots, b_{l}\right)$ is defined to delimit consecutive categories $C_{s}, s=1, \ldots, l$. The vector $\mathbf{b}$ satisfies that $0=b_{0}<b_{1}<\ldots<b_{l}=1+\varepsilon$, where $\varepsilon$ is a small positive number, and $b_{s-1}$ and $b_{s}$ are the lower and upper thresholds of category $C_{s}$, respectively. The classification of alternative $a$ is performed through the comparison between the global value $U(a)$ and the category thresholds $b_{0}, b_{1}, \ldots, b_{l}$, i.e.,

$$
b_{s-1} \leq U(a)<b_{s} \Leftrightarrow a \in C_{s}
$$

Table 1 summarizes the notation used throughout the paper. 
Table 1: Notation.

\begin{tabular}{ll}
\hline Notation & Meaning \\
\hline$A=\left\{a_{1}, a_{2}, \ldots, a_{m}\right\}$ & Set of alternatives to be sorted (i.e., non-reference alternatives). \\
$C=\left\{C_{1}, C_{2}, \ldots, C_{l}\right\}$ & Set of categories. \\
$A^{R}=\left\{a_{1}^{*}, a_{2}^{*}, \ldots, a_{r}^{*}\right\}$ & Set of reference alternatives. \\
$A_{s}^{R}$ & Subset of reference alternatives that are assigned to category $C_{s}$. \\
$r_{s}$ & Cardinality of $A_{s}^{R}$. \\
$a_{i} \in C_{h\left(a_{i}\right)}$ & Alternative $a_{i}$ is assigned to category $C_{h\left(a_{i}\right)}$. \\
$G=\left\{g_{1}, g_{2}, \ldots, g_{n}\right\}$ & Set of criteria. \\
$g_{j}(a)$ & Evaluation of alternative $a$ on criterion $g_{j}$. \\
$U(a)$ & Global value of alternative $a$. \\
$u_{j}(a)$ & Marginal value of alternative $a$ on criterion $g_{j}$. \\
$\mathbf{V}(a)$ & Characteristic vector of alternative $a$. \\
$\mathbf{u}$ & Vector of differences between marginal values of consecutive break-points. \\
$\mathbf{b}=\left(b_{0}, b_{1}, \ldots, b_{l}\right)$ & Vector of category thresholds. \\
$\xi$ & Cluster of reference alternatives coming from a certain category. \\
$P(\xi)$ & Convex hull generated by reference alternatives of cluster $\xi$. \\
$\Theta(P(\xi))$ & Set of vertices of convex hull $P(\xi)$. \\
$\Psi_{s}$ & Set of clusters of reference alternatives from category $C_{s}$ output by Algorithm 1. \\
$\Delta\left(a^{*}, b^{*}\right)$ & Value difference between reference alternatives $a^{*}$ and $b^{*}$. \\
$I\left(a^{*}, b^{*}\right)$ & Dissimilarity between two clusters $\xi_{t}$ and $\xi_{t^{\prime}}$. \\
$\kappa\left(\xi_{t}, \xi_{t}\right)$ & Set of reference alternatives lying in the upper preferential boundary of $\xi_{t}$. \\
$B^{+}\left(\xi_{t}\right)$ & Set of rence alternatives lying in the lower preferential boundary of $\xi_{t}$. \\
\hline$\left.\xi_{t}\right)$ & Value difference between reference alternatives $a^{*}$ and $b^{*}$. \\
\hline
\end{tabular}




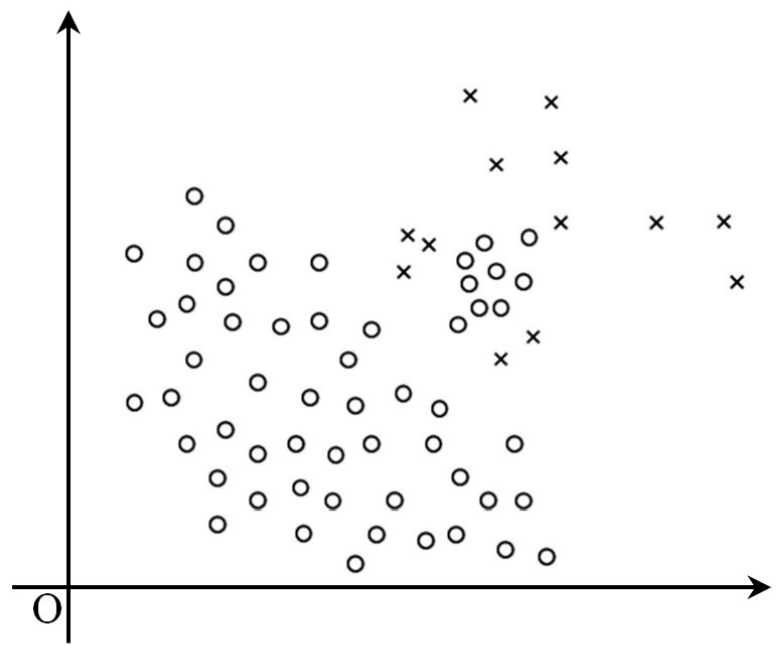

(a)

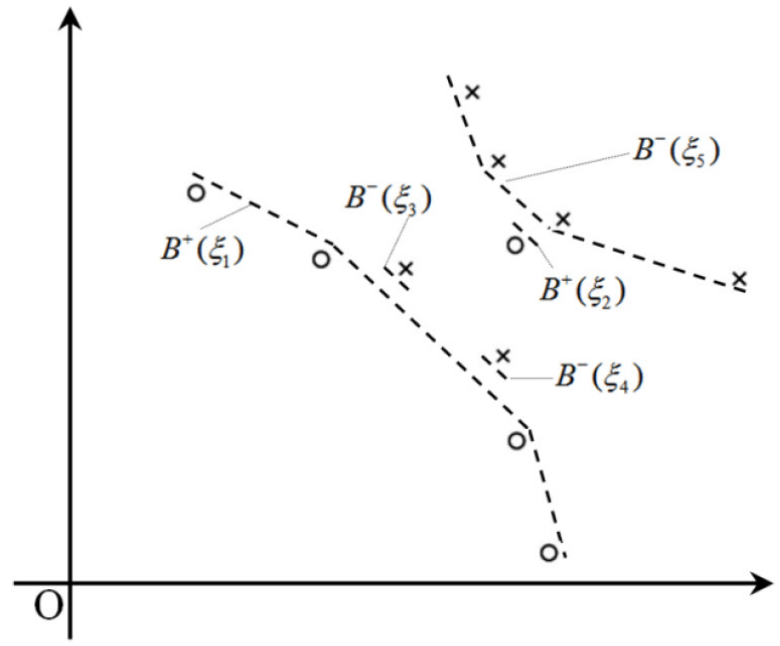

(c)



(b)

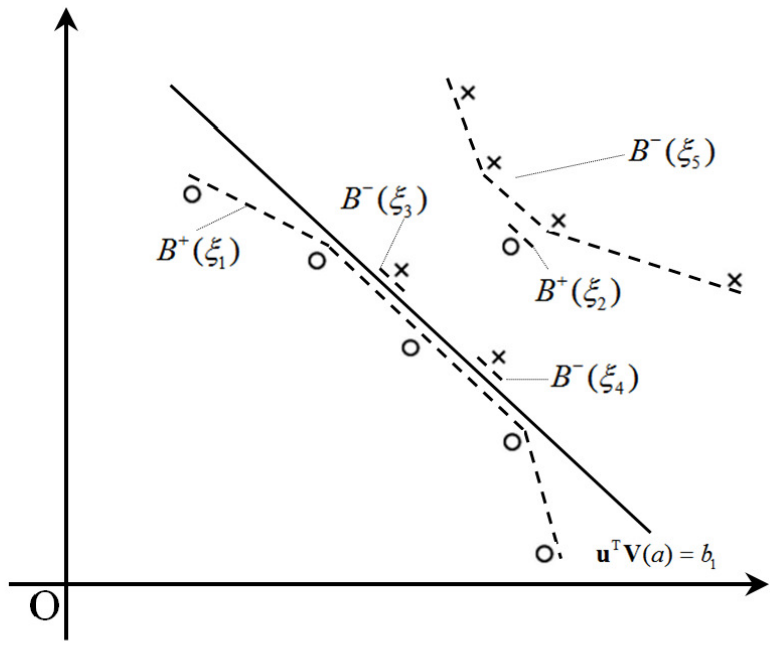

(d)

Figure 1: An illustrative example.

\subsection{Outline of the proposed approach}

We present the geometric ideas of our approach intuitively to facilitate understanding of the mathematical presentation in the manuscript. Let us consider the following example about a MCS problem with an imbalanced set of assignment examples, which is illustrated in Figure 1. Suppose that there are two evaluation criteria $G=\left\{g_{1}, g_{2}\right\}$ and two categories $C=\left\{C_{1}, C_{2}\right\}$. We use a linear additive value function as the preference model and thus characteristic vectors of reference alternatives can be depicted in two-dimensional space. The distribution of characteristic vectors of reference alternatives is illustrated in Figure 1(a), where characteristic vectors of reference alternatives in $C_{1}$ and $C_{2}$ are represented with "o" and "x", respectively. One can observe that $C_{1}$ is the larger category while $C_{2}$ is the smaller one.

Firstly, we utilize the hierarchical clustering algorithm to group reference alternatives into clusters, so that 
each cluster $\xi$ consists of reference alternatives $a_{i}^{*}$ coming from a certain category $C_{s}, s=1, \ldots, l$, and no characteristic vectors $\mathbf{V}\left(a_{j}^{*}\right)$ of reference alternatives $a_{j}^{*}$ from other categories $C_{s^{\prime}}, s^{\prime} \neq s$, lie in the convex hull $P(\xi)$ generated by $\mathbf{V}\left(a_{i}^{*}\right), a_{i}^{*} \in \xi$. Suppose that the clustering result is illustrated in Figure 1(b), in which there are five clusters, i.e., $\xi_{1}, \xi_{2}, \xi_{3}, \xi_{4}$, and $\xi_{5}$, and convex hulls generated by characteristic vectors of reference alternatives from each cluster are depicted in dashed lines.

Then, we propose several LP models to identify reference alternatives lying in preferential boundaries of each cluster, which consists of the upper preferential boundary and the lower one. For any cluster $\xi$, the upper preferential boundary, denote by $B^{+}(\xi)$, and the lower preferential boundary, denote by $B^{-}(\xi)$, are defined as the parts of boundary of $P(\xi)$ that are expanded by characteristic vectors $\mathbf{V}\left(a_{i}^{*}\right)$ of reference alternatives $a_{i}^{*} \in \xi$ whose values could be the maximum and the minimum among all reference alternatives in $\xi$, respectively. Thus, reference alternatives lying in preferential boundaries of each cluster refer to those that could be potentially optimal or worst among the ones of the corresponding cluster. If reference alternatives lying in preferential boundaries of a cluster are correctly classified, then all the others of the corresponding cluster are also correctly classified. Therefore, reference alternatives lying in preferential boundaries of clusters are the ones that are active to develop the sorting model. In this example, we identify the reference alternatives lying in the upper preferential boundaries of $\xi_{1}$ and $\xi_{2}$, denoted by $B^{+}\left(\xi_{1}\right)$ and $B^{+}\left(\xi_{2}\right)$, respectively, and those lying in the lower preferential boundaries of $\xi_{3}, \xi_{4}$, and $\xi_{5}$, denoted by $B^{-}\left(\xi_{3}\right), B^{-}\left(\xi_{4}\right)$, and $B^{-}\left(\xi_{5}\right)$, respectively, which are illustrated in Figure 1(c).

Then, in order to construct a balanced set of assignment examples, a balancing algorithm is proposed to balance active reference alternatives across categories. The algorithm uses an augmenting strategy to iteratively add artificial alternatives until the distribution of active reference alternatives across categories is adjusted to be balanced. In this example, as $\left|B^{+}\left(\xi_{1}\right)\right|+\left|B^{+}\left(\xi_{2}\right)\right|=5$ and $\left|B^{-}\left(\xi_{3}\right)\right|+\left|B^{-}\left(\xi_{4}\right)\right|+\left|B^{-}\left(\xi_{5}\right)\right|=6$, we need to add an artificial alternative to $B^{+}\left(\xi_{1}\right)$ or $B^{+}\left(\xi_{2}\right)$ to obtain a balanced set of assignment examples. Suppose that an artificial alternative is added to $B^{+}\left(\xi_{1}\right)$ and the obtained set of assignment examples is illustrated in Figure 1(d).

Finally, the sorting model is obtained by minimizing the sum of violations between values of active reference alternatives and the corresponding category thresholds. In this example, reference alternatives $a_{i}^{*} \in C_{1}$ should satisfy the constraint of $b_{0} \leq U\left(a_{i}^{*}\right)<b_{1}$, and reference alternatives $a_{i}^{*} \in C_{2}$ should satisfy the constraint of $b_{1} \leq U\left(a_{i}^{*}\right)<b_{2}$. As $b_{0}=0$ and $b_{2}=1+\varepsilon$, the constraints of $U\left(a_{i}^{*}\right) \geq b_{0}$ for $a_{i}^{*} \in C_{1}$ and $U\left(a_{i}^{*}\right)<b_{2}$ for $a_{i}^{*} \in C_{2}$ can always be satisfied. Thus, we just consider the violations between values of active reference alternatives from $C_{1}$ (i.e., $\left.U\left(a_{i}^{*}\right), a_{i}^{*} \in B^{+}\left(\xi_{1}\right), B^{+}\left(\xi_{2}\right)\right)$ and the category threshold $b_{1}$, and those between values of active reference alternatives from $C_{2}$ (i.e., $\left.U\left(a_{i}^{*}\right), a_{i}^{*} \in B^{-}\left(\xi_{3}\right), B^{-}\left(\xi_{4}\right), B^{-}\left(\xi_{5}\right)\right)$ and the category threshold $b_{1}$. Then, the sorting model is obtained by minimizing the sum of these violations, and the hyperplane $\mathbf{u}^{\mathrm{T}} \mathbf{V}(a)=b_{1}$ is illustrated in Figure 1(d).

The process of the proposed approach is summarized as follows.

Step 1. Make use of a hierarchical clustering algorithm to group reference alternatives of each category into clusters (see Section 2.3 for more details). 
Step 2. Solve LP models to identify reference alternatives lying in upper and lower preferential boundaries of each cluster (see Section 2.4 for more details).

Step 3. Use a balancing algorithm to adjust the identified reference alternatives lying in preferential boundaries of clusters in each category to be balanced (see Section 2.5 for more details).

Step 4. Solve a LP model to infer a sorting model by minimizing the violations between the values of reference alternatives lying in preferential boundaries of clusters and the corresponding category thresholds, and then apply the inferred sorting model to assign non-reference alternatives into categories (see Section 2.6 for more details).

\subsection{Grouping reference alternatives into clusters}

In this section, we apply the hierarchical clustering algorithm proposed in [44] to group reference alternatives into clusters, without specification of the number of clusters. The clustering algorithm runs repeatedly for different categories, with each iteration grouping reference alternatives of a certain category into clusters. Each cluster $\xi$ consists of reference alternatives $a_{i}^{*}$ coming from a certain category $C_{s}, s=1, \ldots, l$, and ensures that no characteristic vectors $\mathbf{V}\left(a_{j}^{*}\right)$ of reference alternatives $a_{j}^{*}$ from other categories $C_{s^{\prime}}, s^{\prime} \neq s$, lie in the convex hull $P(\xi)$ generated by $\mathbf{V}\left(a_{i}^{*}\right), a_{i}^{*} \in \xi$.

Let us consider to group reference alternatives of a certain category $C_{s}, s=1, \ldots, l$, into clusters. Initially, each reference alternative $a_{i}^{*} \in A_{s}^{R}$ is considered as an initial cluster $\xi_{i}=\left\{a_{i}^{*}\right\}$. The algorithm iteratively merges two clusters $\xi_{t}, \xi_{t^{\prime}}, t, t^{\prime} \in \mathbb{Z}^{+}$, satisfying that (a) $\xi_{t}$ and $\xi_{t^{\prime}}$ consist of reference alternatives $a_{i}^{*}$ coming from the same category $C_{s}$, and (b) the dissimilarity between $\xi_{t}$ and $\xi_{t^{\prime}}$ is the minimum, to generate a new cluster $\xi_{t^{\prime \prime}}, t^{\prime \prime} \in \mathbb{Z}^{+}$, ensuring that no characterístic vectors $\mathbf{V}\left(a_{j}^{*}\right)$ of reference alternatives $a_{j}^{*}$ from other categories $C_{s^{\prime}}, s^{\prime} \neq s$, lie in the convex hull $P\left(\xi_{t^{\prime \prime}}\right)$ generated by $\mathbf{V}\left(a_{i}^{*}\right), a_{i}^{*} \in \xi_{t^{\prime \prime}}=\xi_{t} \cup \xi_{t^{\prime}}$. To check whether clusters $\xi_{t}, \xi_{t^{\prime}}$ can be merged to generate a new cluster $\xi_{t^{\prime \prime}}$, we need to solve the following LP model for each $a_{j}^{*} \in A_{s^{\prime}}^{R}, s^{\prime}=1, \ldots, s-1, s+1, \ldots, l:$

$$
\begin{aligned}
L P 1: & \varepsilon^{*}\left(a_{j}^{*}\right)=\max \varepsilon, \\
\text { s.t. } & \mathbf{p}^{\mathrm{T}} \mathbf{V}\left(a_{j}^{*}\right)+q \leq-\varepsilon, \\
& \mathbf{p}^{\mathrm{T}} \mathbf{V}\left(a_{i}^{*}\right)+q \geq \varepsilon, \quad a_{i}^{*} \in \xi_{t} \cup \xi_{t^{\prime}}, \\
& 0 \leq \varepsilon \leq 1 .
\end{aligned}
$$

where $\mathbf{p} \in \mathbb{R}^{\gamma}$ and $q \in \mathbb{R}$ are variables used to define a hyperplane. The constraint (12) is added to prevent unbounded solutions. If $\varepsilon^{*}\left(a_{j}^{*}\right)>0$, then the hyperplane $H=\left\{\mathbf{V} \in \mathbb{R}^{\gamma} \mid \mathbf{p}^{* \mathrm{~T}} \mathbf{V}+q^{*}=0\right\}$, where $\mathbf{p}^{*}, q^{*}$ are the values of variables of $L P 1$ at the optimum, separates $\mathbf{V}\left(a_{j}^{*}\right)$ from $\mathbf{V}\left(a_{i}^{*}\right), a_{i}^{*} \in \xi_{t} \cup \xi_{t^{\prime}}$, and thus $\mathbf{V}\left(a_{j}^{*}\right)$ does not lie in $P\left(\xi_{t^{\prime \prime}}\right)$, which is denoted by $\mathbf{V}\left(a_{j}^{*}\right) \notin P\left(\xi_{t^{\prime \prime}}\right)$; otherwise, there is no hyperplane that can separate $\mathbf{V}\left(a_{j}^{*}\right)$ from $\mathbf{V}\left(a_{i}^{*}\right), a_{i}^{*} \in \xi_{t} \cup \xi_{t^{\prime}}$, and thus $\mathbf{V}\left(a_{j}^{*}\right)$ lies in $P\left(\xi_{t^{\prime \prime}}\right)$, which is denoted by $\mathbf{V}\left(a_{j}^{*}\right) \in P\left(\xi_{t^{\prime \prime}}\right)$.

The underlying idea of $L P 1$ can be illustrated in Figure 2. There are two clusters $\xi_{t}=\left\{a_{3}^{*}, a_{4}^{*}, a_{5}^{*}, a_{6}^{*}, a_{7}^{*}\right\}$ and $\xi_{t^{\prime}}=\left\{a_{8}^{*}, a_{9}^{*}, a_{10}^{*}, a_{11}^{*}, a_{12}^{*}, a_{13}^{*}\right\}$ consisting of reference alternatives coming from the same category, and the characteristic vectors of reference alternatives in $\xi_{t}$ and $\xi_{t^{\prime}}$ are represented with "o". Another two reference 
alternatives $a_{1}^{*}$ and $a_{2}^{*}$ belong to other categories and their characteristic vectors are represented with "x". It can be observed that, on one hand, the hyperplane $H_{1}$ separates $\mathbf{V}\left(a_{1}^{*}\right)$ from $\mathbf{V}\left(a_{j}^{*}\right), a_{j}^{*} \in \xi_{t} \cup \xi_{t^{\prime}}$, and thus $\mathbf{V}\left(a_{1}^{*}\right) \notin P\left(\xi_{t^{\prime \prime}}\right)$, where $\xi_{t^{\prime \prime}}$ is the merger of clusters $\xi_{t}$ and $\xi_{t^{\prime}}$; on the other hand, there is no hyperplane that can separate $\mathbf{V}\left(a_{2}^{*}\right)$ from $\mathbf{V}\left(a_{j}^{*}\right), a_{j}^{*} \in \xi_{t} \cup \xi_{t^{\prime}}$, and thus $\mathbf{V}\left(a_{2}^{*}\right) \in P\left(\xi_{t^{\prime \prime}}\right)$.

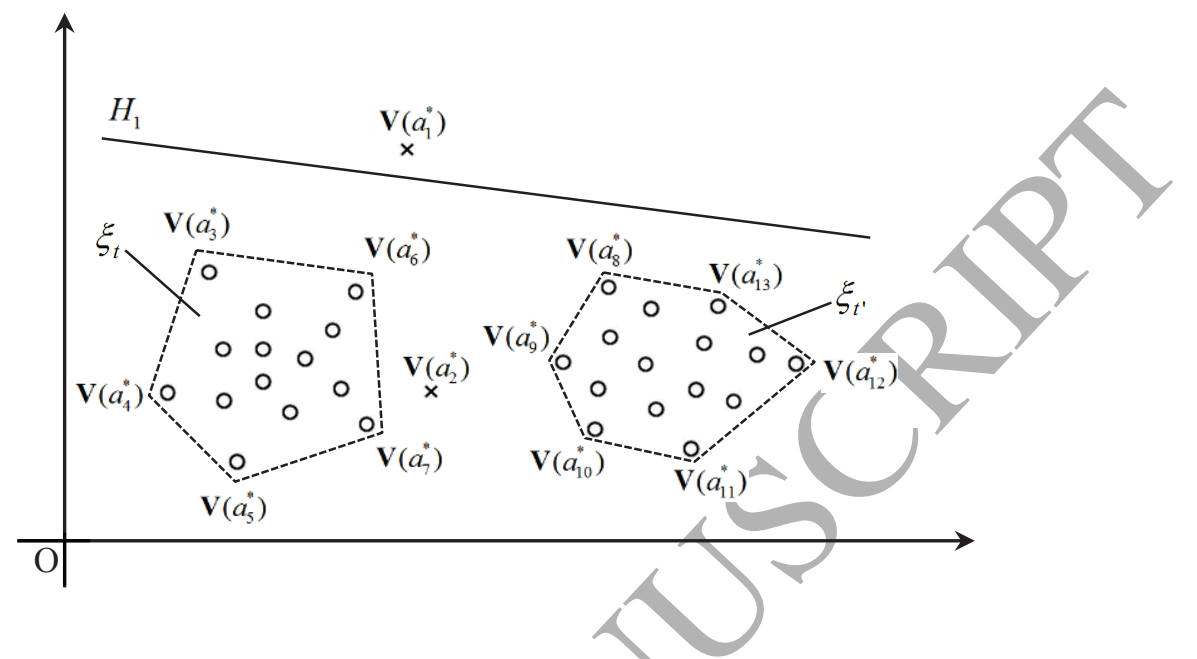

Figure 2: The underlying idea of $L P 1$.

We solve $L P 1$ for all the reference alternatives $a_{j}^{*} \in A_{s^{\prime}}^{R}, s^{\prime}=1, \ldots, s-1, s+1, \ldots, l$. If $\varepsilon^{*}\left(a_{j}^{*}\right)>0$, for all $a_{j}^{*} \in A_{s^{\prime}}^{R}, s^{\prime}=1, \ldots, s-1, s+1, \ldots, l$, then $\xi_{t}$ and $\xi_{t^{\prime}}$ can be merged to generate a new cluster $\xi_{t^{\prime \prime}}$, ensuring that no characteristic vectors of reference alternatives belonging to other categories lie in $P\left(\xi_{t^{\prime \prime}}\right)$.

The overall hierarchical clustering algorithm for grouping reference alternatives $a_{i}^{*} \in A^{R}$ into clusters is described in Algorithm 1. Algorithm 1 applies the hierarchical clustering methodology [45] where clusters with the minimum dissimilarity are merged to generate a new cluster. The clustering result is $\Psi=\left\{\Psi_{1}, \ldots, \Psi_{l}\right\}$ and $\Psi_{s}, s=1, \ldots, l$ is composed of clusters of reference alternatives from category $C_{s}$. Note that the dissimilarity between two clusters in Algorithm 1 is calculated in the following way:

Definition 2. The value difference $\Delta\left(a^{*}, b^{*}\right)$ between two reference alternatives $a^{*}, b^{*} \in A^{R}$ is defined as the difference of $U\left(a^{*}\right)$ and $U\left(b^{*}\right)$, i.e.,

$$
\begin{aligned}
\Delta\left(a^{*}, b^{*}\right) & =U\left(a^{*}\right)-U\left(b^{*}\right) \\
& =\mathbf{u}^{\mathrm{T}} \mathbf{V}\left(a^{*}\right)-\mathbf{u}^{\mathrm{T}} \mathbf{V}\left(b^{*}\right) .
\end{aligned}
$$

Definition 3. The variance of value difference $I\left(a^{*}, b^{*}\right)$ between two reference alternatives $a^{*}, b^{*} \in A^{R}$ is defined as the difference between the maximum and minimum values of $\Delta\left(a^{*}, b^{*}\right)$, i.e.,

$$
I\left(a^{*}, b^{*}\right)=\Delta_{\max }\left(a^{*}, b^{*}\right)-\Delta_{\min }\left(a^{*}, b^{*}\right)
$$

where $\Delta_{\max }\left(a^{*}, b^{*}\right)$ and $\Delta_{\min }\left(a^{*}, b^{*}\right)$ are obtained by solving the following two LP models $L P 2$ and $L P 3$. 


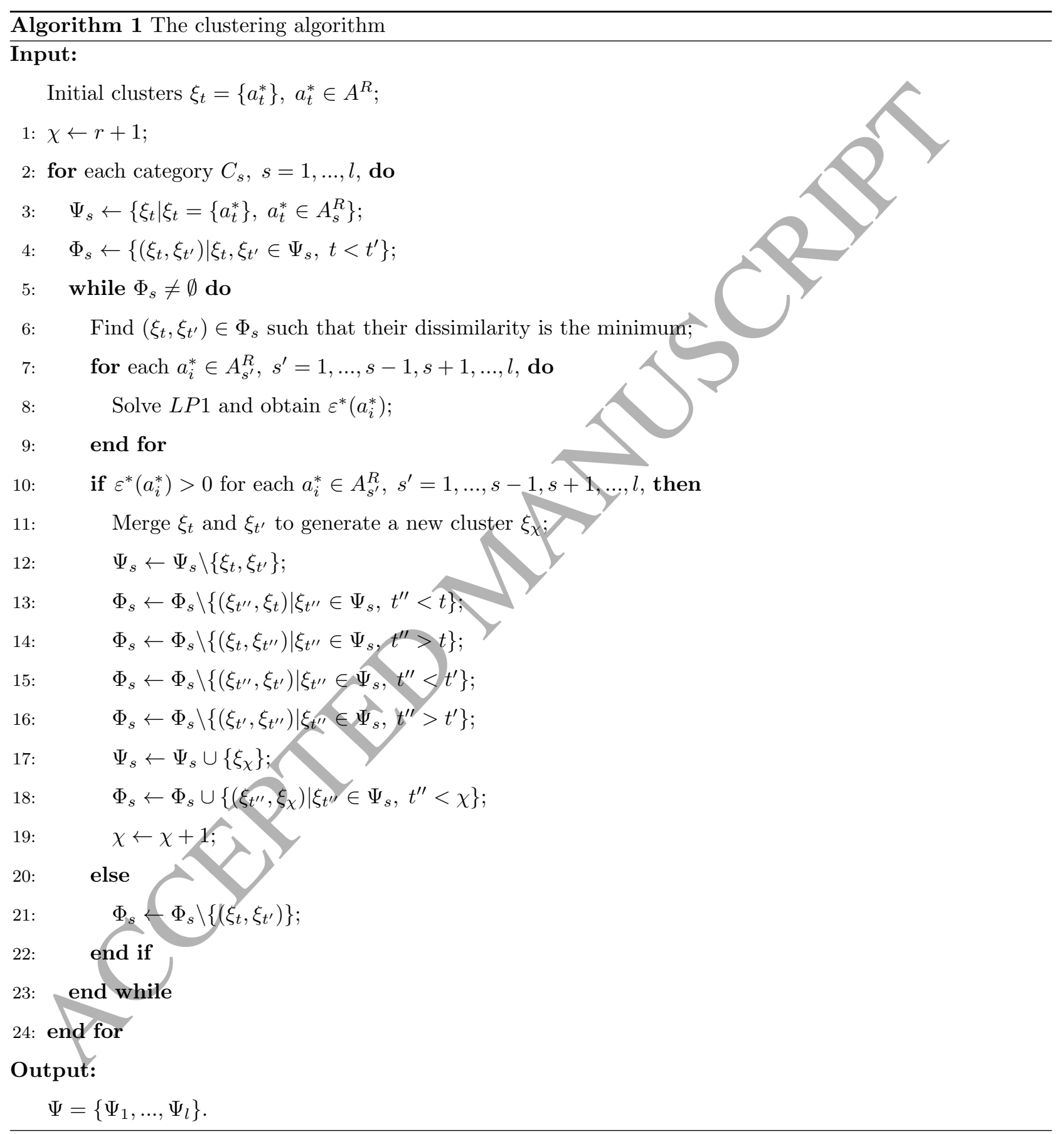




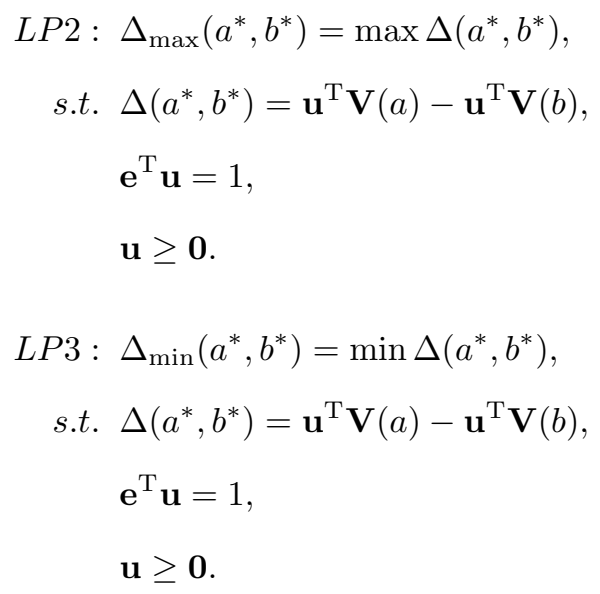

Proposition 1. For any reference alternatives $a^{*}, b^{*} \in A^{R}$, we have $I\left(a^{*}, b^{*}\right)=I\left(b^{*}, a^{*}\right)$.

Proof. Let $\partial$ be the set of $\mathbf{u}$ satisfying the set of constraints of $L P 2$ and $L P 3$.

$$
\begin{aligned}
I\left(a^{*}, b^{*}\right) & =\Delta_{\max }\left(a^{*}, b^{*}\right)-\Delta_{\min }\left(a^{*}, b^{*}\right) \\
& =\max _{\mathbf{u} \in \partial}\left(\mathbf{u}^{\mathrm{T}} \mathbf{V}\left(a^{*}\right)-\mathbf{u}^{\mathrm{T}} \mathbf{V}\left(b^{*}\right)\right)-\min _{\mathbf{u} \in \partial}\left(\mathbf{u}^{\mathrm{T}} \mathbf{V}\left(a^{*}\right)-\mathbf{u}^{\mathrm{T}} \mathbf{V}\left(b^{*}\right)\right) \\
& =-\min _{\mathbf{u} \in \partial}\left(-\left(\mathbf{u}^{\mathrm{T}} \mathbf{V}\left(a^{*}\right)-\mathbf{u}^{\mathrm{T}} \mathbf{V}\left(b^{*}\right)\right)\right)+\max _{\mathbf{u} \in \partial}\left(-\left(\mathbf{u}^{\mathrm{T}} \mathbf{V}\left(a^{*}\right)-\mathbf{u}^{\mathrm{T}} \mathbf{V}\left(b^{*}\right)\right)\right) \\
& =-\min _{\mathbf{u} \in \partial}\left(\mathbf{u}^{\mathrm{T}} \mathbf{V}\left(b^{*}\right)-\mathbf{u}^{\mathrm{T}} \mathbf{V}\left(a^{*}\right)\right)+\max _{\mathbf{u} \in \partial}\left(\mathbf{u}^{\mathrm{T}} \mathbf{V}\left(b^{*}\right)-\mathbf{u}^{\mathrm{T}} \mathbf{V}\left(a^{*}\right)\right) \\
& \left.=\max _{\mathbf{u} \in \partial}\left(\mathbf{u}^{\mathrm{T}} \mathbf{V}\left(b^{*}\right)-\mathbf{u}^{\mathrm{T}} \mathbf{V}\left(a^{*}\right)\right)-\min _{\mathbf{u} \in \partial} \mathbf{u}^{\mathrm{T}} \mathbf{V}\left(b^{*}\right)-\mathbf{u}^{\mathrm{T}} \mathbf{V}\left(a^{*}\right)\right) \\
& =I\left(b^{*}, a^{*}\right) .
\end{aligned}
$$

Definition 4. The dissimilarity $\kappa\left(\xi_{t}, \xi_{t^{\prime}}\right)$ between two clusters $\xi_{t}, \xi_{t^{\prime}}$ is defined as

$$
\kappa\left(\xi_{t}, \xi_{t^{\prime}}\right)=\frac{\sum_{a^{*} \in \xi_{t}, b^{*} \in \xi_{t^{\prime}}} I\left(a^{*}, b^{*}\right)}{2 \times\left|\xi_{t}\right| \times\left|\xi_{t^{\prime}}\right|} .
$$

As $\Delta\left(a^{*}, b^{*}\right) \in[-1,1]$ and $I\left(a^{*}, b^{*}\right), \in[0,2]$, thus $\kappa\left(\xi_{t}, \xi_{t^{\prime}}\right)$ is normalized in $[0,1]$. Definition 4 measures the dissimilarity between two clusters from the view of multiple criteria analysis.

\subsection{Identifying reference alternatives lying in preferential boundaries of clusters}

In this section, we propose a method to identify reference alternatives lying in preferential boundaries of clusters, which are the ones that are active to develop the sorting model.

Definition 5. [46]. Let $P \subset \mathbb{R}^{\gamma}$ be a polyhedron. A vector $\mathbf{V} \in P$ is a vertex of $P$ if there exists a vector $\mathbf{p} \in \mathbb{R}^{\gamma}$ such that $\mathbf{p}^{\mathrm{T}} \mathbf{V}<\mathbf{p}^{\mathrm{T}} \mathbf{V}^{\prime}$ for all $\mathbf{V}^{\prime}$ satisfying $\mathbf{V}^{\prime} \in P$ and $\mathbf{V}^{\prime} \neq \mathbf{V}$.

Let $\Theta\left(P\left(\xi_{t}\right)\right)$ denote the set of vertices of polyhedron $P\left(\xi_{t}\right)$. For each $a_{i}^{*} \in \xi_{t}$, we propose the following LP model to check whether $\mathbf{V}\left(a_{i}^{*}\right) \in \Theta\left(P\left(\xi_{t}\right)\right)$ :

$$
\begin{aligned}
L P 4: & \varepsilon^{*}\left(a_{i}^{*}\right)=\max \varepsilon, \\
\text { s.t. } & \mathbf{p}^{\mathrm{T}} \mathbf{V}\left(a_{i}^{*}\right)+\varepsilon \leq \mathbf{p}^{\mathrm{T}} \mathbf{V}\left(a_{j}^{*}\right), \quad a_{j}^{*} \in \xi_{t} \backslash\left\{a_{i}^{*}\right\}, \\
& 0 \leq \varepsilon \leq 1,
\end{aligned}
$$


where $\mathbf{p} \in \mathbb{R}^{\gamma}$ and $\varepsilon \in \mathbb{R}$ are variables.

Proposition 2. For any $a_{i}^{*} \in \xi_{t}$, if $\varepsilon^{*}\left(a_{i}^{*}\right)>0$ in $L P 4$, then $\mathbf{V}\left(a_{i}^{*}\right)$ is a vertex of $P\left(\xi_{t}\right)$, i.e., $\mathbf{V}\left(a_{i}^{*}\right) \in \Theta\left(P\left(\xi_{t}\right)\right)$.

Proof. Because $\varepsilon^{*}\left(a_{i}^{*}\right)>0$, there exists a vector $\mathbf{p} \in \mathbb{R}^{\gamma}$ such that $\mathbf{p}^{\mathrm{T}} \mathbf{V}\left(a_{i}^{*}\right)+\varepsilon^{*}\left(a_{i}^{*}\right) \leq \mathbf{p}^{\mathrm{T}} \mathbf{V}\left(a_{j}^{*}\right), a_{j}^{*} \in \xi_{t} \backslash\left\{a_{i}^{*}\right\}$, i.e., $\mathbf{p}^{\mathrm{T}} \mathbf{V}\left(a_{i}^{*}\right)<\mathbf{p}^{\mathrm{T}} \mathbf{V}\left(a_{j}^{*}\right), a_{j}^{*} \in \xi_{t} \backslash\left\{a_{i}^{*}\right\}$. For $P\left(\xi_{t}\right)$ is convex, any vector $\mathbf{V}^{\prime} \in P\left(\xi_{t}\right)$, different from $\mathbf{V}\left(a_{i}^{*}\right)$, can be expressed as $\mathbf{V}^{\prime}=\sum_{a_{j}^{*} \in \xi_{t}} \lambda\left(a_{j}^{*}\right) \mathbf{V}\left(a_{j}^{*}\right)$ where $\sum_{a_{j}^{*} \in \xi_{t}} \lambda\left(a_{j}^{*}\right)=1$ and $\lambda\left(a_{j}^{*}\right) \geq 0, a_{j}^{*} \in \xi_{t}$. Thus, we have $\mathbf{p}^{\mathrm{T}} \mathbf{V}^{\prime}=\mathbf{p}^{\mathrm{T}}\left(\sum_{a_{j}^{*} \in \xi_{t}} \lambda\left(a_{j}^{*}\right) \mathbf{V}\left(a_{j}^{*}\right)\right)=\sum_{a_{j}^{*} \in \xi_{t}} \lambda\left(a_{j}^{*}\right) \mathbf{p}^{\mathrm{T}} \mathbf{V}\left(a_{j}^{*}\right)>\sum_{a_{j}^{*} \in \xi_{t}} \lambda\left(a_{j}^{*}\right) \mathbf{p}^{\mathrm{T}} \mathbf{V}\left(a_{i}^{*}\right)=\mathbf{p}^{\mathrm{T}} \mathbf{V}\left(a_{i}^{*}\right)$. According to Definition $5, \mathbf{V}\left(a_{i}^{*}\right)$ is a vertex of $P\left(\xi_{t}\right)$, i.e., $\mathbf{V}\left(a_{i}^{*}\right) \in \Theta\left(P\left(\xi_{t}\right)\right)$.

Proposition 3. For any vector $\mathbf{V} \in P\left(\xi_{t}\right)$, if $\mathbf{V} \in \Theta\left(P\left(\xi_{t}\right)\right)$, then $\mathbf{V} \in\left\{\mathbf{V}\left(a_{i}^{*}\right) \mid a_{i}^{*} \in \xi_{t}\right\}$.

Proof. Suppose that $\mathbf{V} \notin\left\{\mathbf{V}\left(a_{i}^{*}\right) \mid a_{i}^{*} \in \xi_{t}\right\}$. Thus, $\mathbf{V}$ must be different from $\mathbf{V}\left(a_{i}^{*}\right), a_{i}^{*} \in \xi_{t}$. On one hand, because $P\left(\xi_{t}\right)$ is convex, $\mathbf{V}$ can be expressed as a convex combination of vectors $\boldsymbol{V}\left(a_{i}^{*}\right), a_{i}^{*} \in \xi_{t}$, i.e., $\mathbf{V}=\sum_{a_{i}^{*} \in \xi_{t}} \lambda\left(a_{i}^{*}\right) \mathbf{V}\left(a_{i}^{*}\right)$ where $\sum_{a_{i}^{*} \in \xi_{t}} \lambda_{a_{i}^{*}}=1$ and $\lambda_{a_{i}^{*}} \geq 0, a_{i}^{*} \in \xi_{t}$. On the other hand, according to [46], vertex $\mathbf{V}$ is also an extreme point of $P\left(\xi_{t}\right)$, which cannot be expressed as a convex combination of different vectors. The two sides contradict each other. Thus, the assumption does not hold and it must be that $\mathbf{V} \in\left\{\mathbf{V}\left(a_{i}^{*}\right) \mid a_{i}^{*} \in \xi_{t}\right\}$.

According to Propositions 2 and 3, we have $\Theta\left(P\left(\xi_{t}\right)\right)=\left\{\mathbf{V}\left(a_{i}^{*}\right) \mid \varepsilon^{*}\left(a_{i}^{*}\right)>0, a_{i}^{*} \in \xi_{t}\right\}$ where $\varepsilon^{*}\left(a_{i}^{*}\right)$ is the optimal value of the objective function of $L P 4$.

Proposition 4. For any cluster $\xi_{t} \in \Psi_{s}, s=1, \ldots, l$, and any reference alternative $a_{i}^{*} \in \xi_{t}$, we have

$$
\min _{\mathbf{V}\left(a_{j}^{*}\right) \in \Theta\left(P\left(\xi_{t}\right)\right)} U\left(a_{j}^{*}\right) \leq U\left(a_{i}^{*}\right) \leq \max _{\mathbf{V}\left(a_{j}^{*}\right) \in \Theta\left(P\left(\xi_{t}\right)\right)} U\left(a_{j}^{*}\right) .
$$

Proof. Let us consider the following LP problem: $\max U=\mathbf{u}^{\mathrm{T}} \mathbf{V}$, s.t., $\mathbf{V} \in P\left(\xi_{t}\right)$, where $\mathbf{u}$ is fixed. As we know, the optimal solution of an LP problem can be obtained from one of the vertices of the feasible region. Thus, a particular vertex $\mathbf{V}\left(a_{j^{*}}^{*}\right) \in \Theta\left(P\left(\xi_{t}\right)\right), a_{j^{*}}^{*} \in \xi_{t}$, corresponding to the maximum value of the objective function of the above $L P$ model, i.e., $\max _{\left(a_{j}^{*}\right) \in \Theta\left(P\left(\xi_{t}\right)\right)} U\left(a_{j}^{*}\right)=U\left(a_{j^{*}}^{*}\right)=\mathbf{u}^{\mathrm{T}} \mathbf{V}\left(a_{j^{*}}^{*}\right)$. On the other hand, because $a_{i}^{*} \in \xi_{t}$, thus $\mathbf{V}\left(a_{i}^{*}\right) \in P\left(\xi_{t}\right)$ and $U\left(a_{i}^{*}\right) \leq \max _{\mathbf{V}\left(a_{j}^{*}\right) \in \Theta\left(P\left(\xi_{t}\right)\right)} U\left(a_{j}^{*}\right)$. Analogously, we can prove that $U\left(a_{i}^{*}\right) \geq \min _{\mathbf{V}\left(a_{j}^{*}\right) \in \Theta\left(P\left(\xi_{t}\right)\right)} U\left(a_{j}^{*}\right)$. Thus, we have $\min _{\mathbf{V}\left(a_{j}^{*}\right) \in \Theta\left(P\left(\xi_{t}\right)\right)} U\left(a_{j}^{*}\right) \leq U\left(a_{i}^{*}\right) \leq \max _{\mathbf{V}\left(a_{j}^{*}\right) \in \Theta\left(P\left(\xi_{t}\right)\right)} U\left(a_{j}^{*}\right)$.

Proposition 4 states that for any cluster, the maximum and minimum values of the reference alternatives in the cluster can be found in the vertices of the polyhedron generated by the cluster.

Definition 6. Let $\mathcal{U}=\left\{\mathbf{u} \mid \mathbf{e}^{\mathrm{T}} \mathbf{u}=1, \mathbf{u} \geq 0\right\}$. For any cluster $\xi_{t} \in \Psi_{s}, s=1, \ldots, l$, (a) the upper preferential boundary of $\xi_{t}$ is defined as the part of boundary of $P\left(\xi_{t}\right)$ that is expanded by characteristic vectors $\mathbf{V}\left(a^{*}\right)$ of reference alternatives $a^{*} \in \xi_{t}$ whose values could be the maximum among all reference alternatives in $\xi_{t}$ for at least one vector $\mathbf{u} \in \mathcal{U}$; (b) the lower preferential boundary of $\xi_{t}$ is defined as the part of boundary of $P\left(\xi_{t}\right)$ that is expanded by characteristic vectors $\mathbf{V}\left(a^{*}\right)$ of reference alternatives $a^{*} \in \xi_{t}$ whose values could be the minimum among all reference alternatives in $\xi_{t}$ for at least one vector $\mathbf{u} \in \mathcal{U}$.

According to Proposition 4, the reference alternatives lying in preferential boundaries of $\xi_{t}$ can be found in the vertices of the polyhedron generated by $\xi_{t}$. Let $B^{+}\left(\xi_{t}\right)$ and $B^{-}\left(\xi_{t}\right)$ denote the set of reference alternatives 
lying in the upper and lower preferential boundaries of $\xi_{t}$, respectively. For any reference alternative $a_{i}^{*} \in \xi_{t}$ such that $\mathbf{V}\left(a_{i}^{*}\right) \in \Theta\left(P\left(\xi_{t}\right)\right)$, we can solve the following LP models to check whether $a_{i}^{*} \in B^{+}\left(\xi_{t}\right)$ or $a_{i}^{*} \in B^{-}\left(\xi_{t}\right)$.

$$
\begin{aligned}
\text { LP5: } & \varepsilon\left(a_{i}^{*}\right)=\max \varepsilon, \\
\text { s.t. } \quad & \mathbf{u}^{\mathrm{T}} \mathbf{V}\left(a_{i}^{*}\right) \geq \mathbf{u}^{\mathrm{T}} \mathbf{V}\left(a_{j}^{*}\right)+\varepsilon, \quad a_{j}^{*} \in \xi_{t}, i \neq j, \\
& \mathbf{e}^{\mathrm{T}} \mathbf{u}=1, \\
& \mathbf{u} \geq \mathbf{0} . \\
\text { LP6: } & \varepsilon\left(a_{i}^{*}\right)=\max \varepsilon, \\
\text { s.t. } & \mathbf{u}^{\mathrm{T}} \mathbf{V}\left(a_{i}^{*}\right) \leq \mathbf{u}^{\mathrm{T}} \mathbf{V}\left(a_{j}^{*}\right)-\varepsilon, a_{j}^{*} \in \xi_{t}, i \neq j, \\
& \mathbf{e}^{\mathrm{T}} \mathbf{u}=1, \\
& \mathbf{u} \geq \mathbf{0} .
\end{aligned}
$$

In $L P 5$, if $\varepsilon\left(a_{i}^{*}\right) \geq 0, a_{i}^{*} \in B^{+}\left(\xi_{t}\right)$; otherwise, $a_{i}^{*} \notin B^{+}\left(\xi_{t}\right)$. Analogously, in $L P 6$, if $\varepsilon\left(a_{i}^{*}\right) \geq 0, a_{i}^{*} \in B^{-}\left(\xi_{t}\right)$; otherwise, $a_{i}^{*} \notin B^{-}\left(\xi_{t}\right)$. If reference alternatives in $B^{+}\left(\xi_{t}\right)$ and $B^{-}\left(\xi_{t}\right)$ are correctly classified, then all the other reference alternatives in $\xi_{t}$ are also correctly classified. Thus, reference alternatives lying in preferential boundaries of clusters are the ones that are active to develop the sorting model.

\subsection{Constructing balanced reference alternatives lying in preferential boundaries of clusters}

In this section, in order to construct a balanced set of assignment examples, we propose a balancing algorithm to balance reference alternatives lying in preferential boundaries of clusters. The algorithm uses an augmenting strategy to iteratively add artificial alternatives until the distribution of active reference alternatives across categories is adjusted to be balanced.

Let $N=\max \left\{\max _{s=1, \ldots, l-1} \sum_{\xi_{t} \in \Psi_{s}}\left|B^{+}\left(\xi_{t}\right)\right|, \max _{s=2 \ldots, l} \sum_{\xi_{t} \in \Psi_{s}}\left|B^{-}\left(\xi_{t}\right)\right|\right\}$ denote the number of adjusted reference alternatives lying in upper or lower preferential boundaries of clusters in each category output by the balancing algorithm. That is to say, for each category $C_{s}, s=1, \ldots, l$, the balancing algorithm aims to add artificial alternatives to $B^{+}\left(\xi_{t}\right)$ and $B^{-}\left(\xi_{t}\right), \xi_{t} \in \Psi_{s}$, so that $\sum_{\xi_{t} \in \Psi_{s}}\left|B^{+}\left(\xi_{t}\right)\right|, s=1, \ldots, l-1$, and $\sum_{\xi_{t} \in \Psi_{s}}\left|B^{-}\left(\xi_{t}\right)\right|, s=2, \ldots, l$, increases to $N$ after adjustment. Note that $N$ is fixed. Moreover, we do not consider the reference alternatives lying in the upper preferential boundaries of clusters in $C_{l}$ and the reference alternatives lying in the lower preferential boundaries of clusters in $C_{1}$, as the constraint of $U\left(a^{*}\right)<b_{l}=1+\varepsilon$ for $a^{*} \in A_{l}^{R}$ and the constraint of $U\left(a^{*}\right) \geq b_{0}=0$ for $a^{*} \in A_{1}^{R}$ can always be satisfied.

Let $M=\min \left\{\min _{s=1, \ldots, l-1} \sum_{\xi_{t} \in \Psi_{s}}\left|B^{+}\left(\xi_{t}\right)\right|, \min _{s=2 \ldots, l} \sum_{\xi_{t} \in \Psi_{s}}\left|B^{-}\left(\xi_{t}\right)\right|\right\}$. In each iteration of the balancing algorithm, two reference alternatives $a_{i}^{*}, a_{j}^{*}$ satisfying the following four conditions from a category $C_{s}$ such that $\sum_{\xi_{t} \in \Psi_{s}}\left|B^{+}\left(\xi_{t}\right)\right|=M<N$ (Note that the case of $\sum_{\xi_{t} \in \Psi_{s}}\left|B^{-}\left(\xi_{t}\right)\right|=M<N$ can be analyzed analogously) are 
selected to create a new artificial reference alternative $a_{k}^{*}$ satisfying $\mathbf{V}\left(a_{k}^{*}\right)=\frac{1}{2}\left(\mathbf{V}\left(a_{i}^{*}\right)+\mathbf{V}\left(a_{j}^{*}\right)\right)$ :

$$
\begin{aligned}
& \text { (a) } i<j \\
& \text { (b) } a_{i}^{*}, a_{j}^{*} \in B^{+}\left(\xi_{t}\right), \xi_{t} \in \Psi_{s} \\
& \text { (c) } \varepsilon^{*}\left(a_{i}^{*}, a_{j}^{*}\right)>0 \text { for the following LP model } L P 7 \text {, if }\left|\xi_{t}\right|>2 \text {; } \\
& \text { (d) } I\left(a_{i}^{*}, a_{j}^{*}\right) \text { is the maximum among all } a_{i^{\prime}}^{*}, a_{j^{\prime}}^{*} \text { satisfying (a),(b),(d). }
\end{aligned}
$$

Condition (b) ensures that $a_{i}^{*}, a_{j}^{*}$ are in the set of reference alternatives lying in the upper preferential boundary of the same cluster $\xi_{t}$. Condition (c) guarantees that the line through $\mathbf{V}\left(a_{i}^{*}\right)$ and $\mathbf{V}\left(a_{j}^{*}\right)$ is located at the upper preferential boundary of $\xi_{t}$ so that the created artificial reference alternative $a_{k}^{*}$ will be also located at the boundary.

$$
\begin{aligned}
& \text { LP7: } \varepsilon^{*}\left(a_{i}^{*}, a_{j}^{*}\right)=\max \varepsilon, \\
& \text { s.t. } \mathbf{p}^{\mathrm{T}} \mathbf{V}\left(a_{i}^{*}\right)+q=0 \text {, } \\
& \mathbf{p}^{\mathrm{T}} \mathbf{V}\left(a_{j}^{*}\right)+q=0 \\
& \mathbf{p}^{\mathrm{T}} \mathbf{V}\left(a_{t}^{*}\right)+q \geq 0, \quad a_{t}^{*} \in \xi_{t} \backslash\left\{a_{i}^{*}, a_{j}^{*}\right\}, \\
& \mathbf{p}^{\mathrm{T}} \mathbf{V}\left(a_{t}^{*}\right)+q \leq \varepsilon, \quad a_{t}^{*} \in \xi_{t} \backslash\left\{a_{i}^{*}, a_{j}^{*}\right\}, \\
& \mathbf{p}^{\mathrm{T}} \mathbf{V}\left(a_{t}^{*}\right)+q \geq \varepsilon-v\left(a_{t}^{*}\right), \quad a_{t}^{*} \in \xi_{t} \backslash\left\{a_{i}^{*}, a_{j}^{*}\right\}, \\
& \sum_{a_{t}^{*} \in \xi_{t} \backslash\left\{a_{i}^{*}, a_{j}^{*}\right\}} v\left(a_{t}^{*}\right)=\left|\xi_{t} \backslash\left\{a_{i}^{*}, a_{j}^{*}\right\}\right|-1, \\
& \widehat{y}\left(a_{t}^{*}\right) \in\{0,1\}, a_{t}^{*} \in \xi_{t} \backslash\left\{a_{i}^{*}, a_{j}^{*}\right\}, \\
& 0 \leq \varepsilon \leq 1 \text {. }
\end{aligned}
$$

where $\mathbf{p} \in \mathbb{R}^{\gamma}$ and $q, \varepsilon \in \mathbb{R}$ and $v\left(a_{t}^{*}\right) \in\{0,1\}$ are variables. $L P 7$ checks whether the line through $\mathbf{V}\left(a_{i}^{*}\right)$ and $\mathbf{V}\left(a_{j}^{*}\right)$ is located at the preferential boundary of $\xi_{t}$. If $\varepsilon^{*}\left(a_{i}^{*}, a_{j}^{*}\right)>0$, then $\mathbf{V}\left(a_{i}^{*}\right)$ and $\mathbf{V}\left(a_{j}^{*}\right)$ are located at the hyperplane $H=\left\{\mathbf{V} \in \mathbb{R}^{\gamma} \mid \mathbf{p}^{* \mathrm{~T}} \mathbf{V}+q^{*}=0\right\}$, where $\mathbf{p}^{*}, q^{*}$ are the values of the variables of $L P 7$ at the optimum, and $\mathbf{V}\left(a_{t}^{*}\right)$ of $a_{t}^{*} \in \xi_{t} \backslash\left\{a_{i}^{*}, a_{j}^{*}\right\}$ is located at the hyperplane $H$ or at the side $H^{\prime}=\left\{\mathbf{V} \in \mathbb{R}^{\gamma} \mid \mathbf{p}^{* \mathrm{~T}} \mathbf{V}+q^{*}>0\right\}$, and therefore, the line through $\mathbf{V}\left(a_{i}^{*}\right)$ and $\mathbf{V}\left(a_{j}^{*}\right)$ is located at the preferential boundary of $\xi_{t}$; otherwise, the line through $\mathbf{V}\left(a_{i}^{*}\right)$ and $\mathbf{V}\left(a_{j}^{*}\right)$ is located at the inner of the polyhedron $P\left(\xi_{t}\right)$. Constraints (21)-(24) are used to identify the maximum value of $\mathbf{p}^{\mathrm{T}} \mathbf{V}\left(a_{t}^{*}\right)+q$ of $a_{t}^{*} \in \xi_{t} \backslash\left\{a_{i}^{*}, a_{j}^{*}\right\}$.

The case for lower preferential boundary can be solved analogously. The balancing algorithm is described in Algorithm 2. 


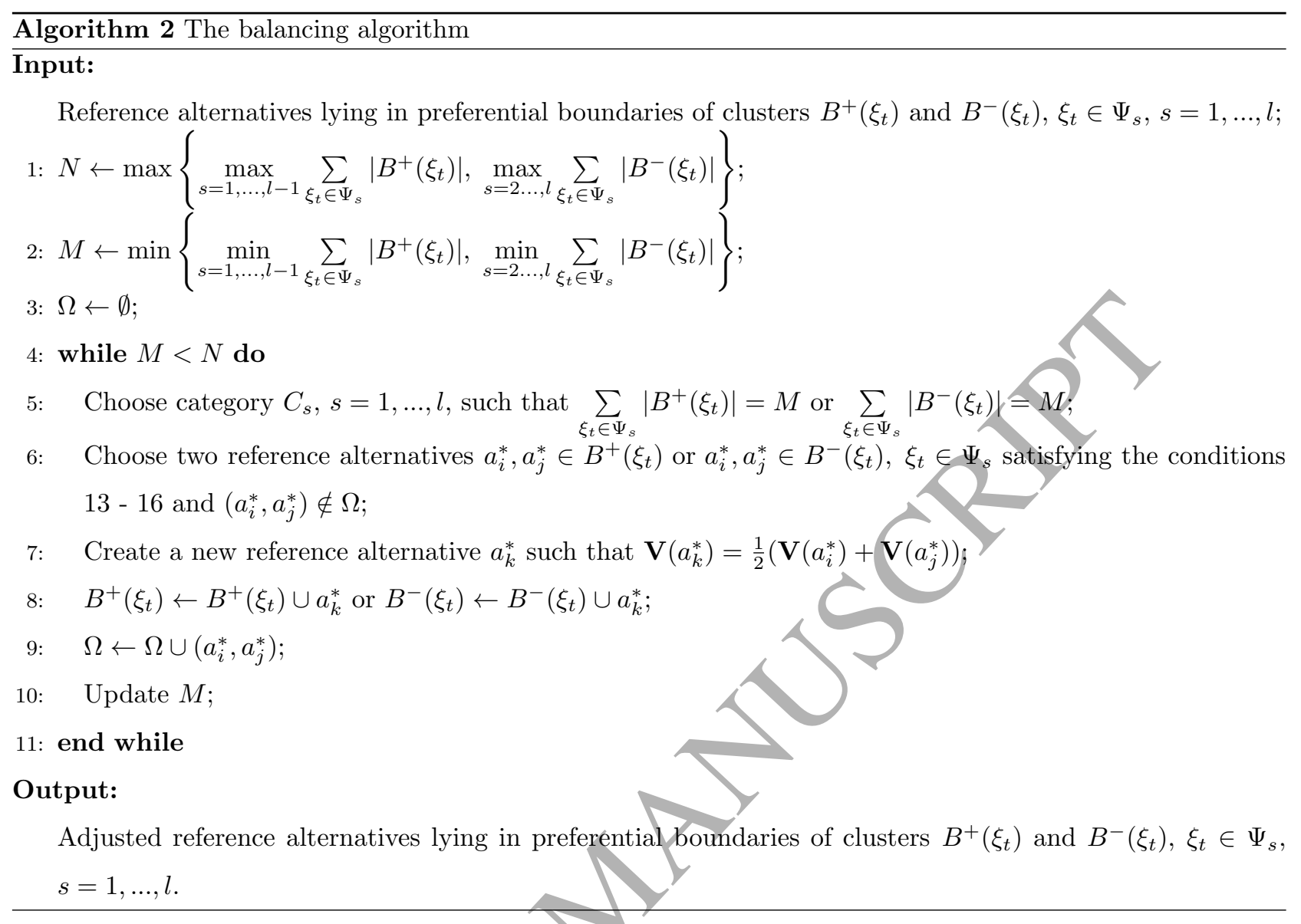

2.6. Inferring a sorting model from reference alternatives lying in preferential boundaries of clusters

In this section, a LP model LP8 is proposed to infer a sorting model by minimizing the sum of violations between values of reference alternatives and the corresponding category thresholds.

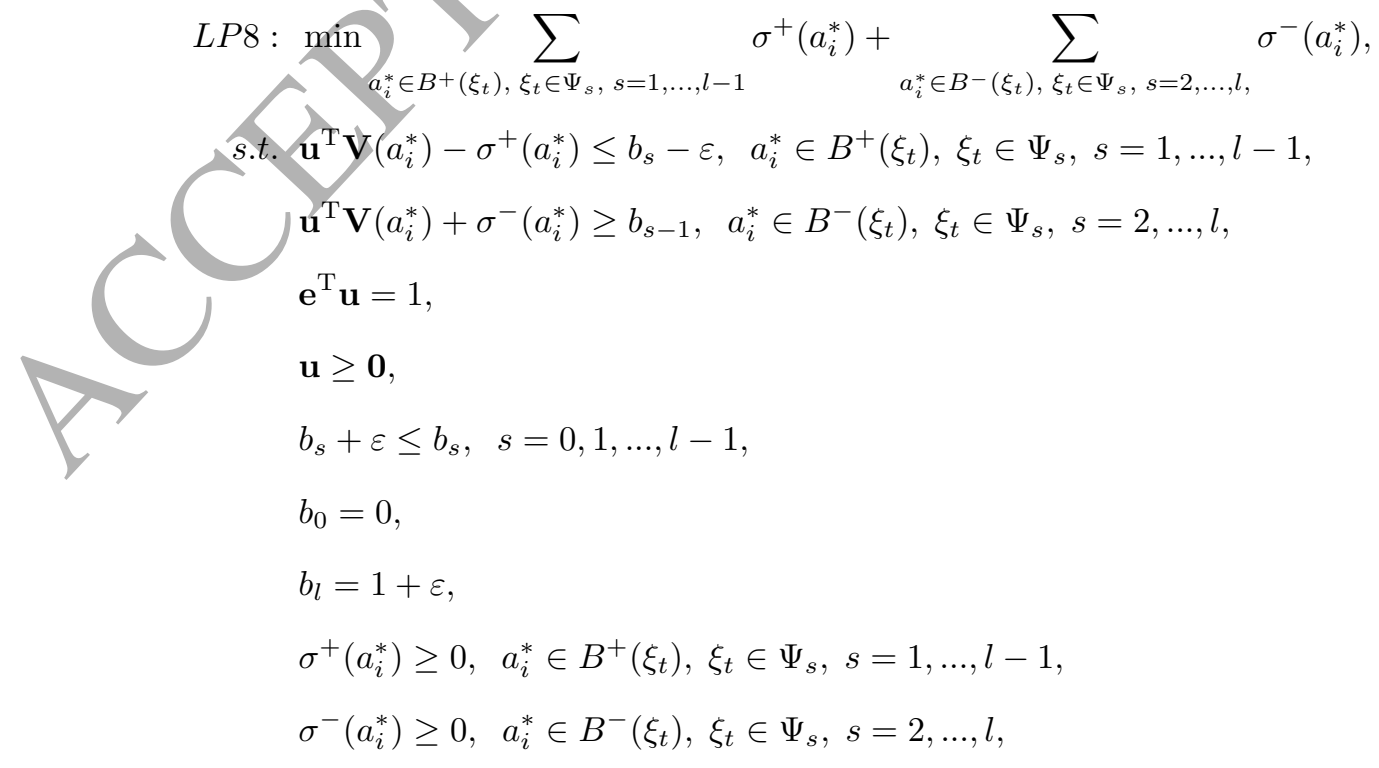


where $\varepsilon$ is a small positive number. For reference alternative $a_{i}^{*}$, the variables $\sigma^{+}\left(a_{i}^{*}\right)$ and $\sigma^{-}\left(a_{i}^{*}\right)$ represent the violations between $U\left(a_{i}^{*}\right)$ and the upper threshold $b_{s}$ and the lower threshold $b_{s-1}$, respectively. As the set of reference alternatives lying in preferential boundaries of clusters in each category are adjusted to be balanced via the balancing algorithm, the LP model $L P 8$ can account for classification accuracy of each category equally and avoid generating biased results across categories.

Let $\mathbf{u}^{*}$ and $\mathbf{b}^{*}$ be the optimal solutions of $L P 8$. With the inferred parameters $\mathbf{u}^{*}$ and $\mathbf{b}^{*}$, we can apply the sorting rule (8) to obtain the assignment for non-reference alternatives $a \in A$, i.e.,

$$
b_{s-1}^{*} \leq U(a)=\mathbf{u}^{* \mathrm{~T}} \mathbf{V}(a)<b_{s}^{*} \Rightarrow a \in C_{s} .
$$

\subsection{Complexity analysis}

In the phase of the clustering analysis (i.e., Step 1 ), we need to solve at most $\sum_{s=1}^{l}\left(r_{s}-1\right)\left(r-r_{s}\right)$ LP model $L P 1$ and $\sum_{s=1}^{l}\left(\begin{array}{l}r_{s} \\ 2\end{array}\right)$ LP models $L P 2$, and $\sum_{s=1}^{l}\left(\begin{array}{l}r_{s} \\ 2\end{array}\right)$ LP models LP3. In the phase of identifying reference alternatives lying in upper and lower preferential boundaries of each cluster (i.e., Step 2), we need to solve at $\operatorname{most} \sum_{s=1}^{l} \sum_{\xi \in \Psi_{s}}|\xi|$ LP model $L P 4, \sum_{s=1}^{l} \sum_{\xi \in \Psi_{s}}|\xi|$ LP model $L P 5$, and $\sum_{s=1}^{l} \sum_{\xi \in \Psi_{s}}|\xi|$ LP model $L P 6$. In the phase of applying the balancing algorithm (i.e., Step 3 ), we need to solve at most $\sum_{s=1}^{l} \sum_{\xi \in \Psi_{s}}\left(\begin{array}{l}|\xi| \\ 2\end{array}\right)$ LP model LP7. The phase of inferring a sorting model (i.e., Step 4 ) consists of solving only one LP model (i.e., $L P 8)$. Assume that the complexity of solving a single $\mathrm{LP}$ model is $O(1)$, and as a result, the computational complexity of the proposed approach is

$$
\begin{aligned}
& \left\{\sum_{s=1}^{l}\left(r_{s}-1\right)\left(r-r_{s}\right)+2 \sum_{s=1}^{l}\left(\begin{array}{l}
r_{s} \\
2
\end{array}\right)+3 \sum_{s=1}^{l} \sum_{\xi \in \Psi_{s}}|\xi|+\sum_{s=1}^{l} \sum_{\xi \in \Psi_{s}}\left(\begin{array}{l}
|\xi| \\
2
\end{array}\right)+1\right\} O(1) \\
& \leq\left\{r^{2}+2 r^{2}+3 r+r^{2}+1\right\} O(1)=\left\{4 r^{2}+3 r+1\right\} O(1)=O\left(r^{2}\right) .
\end{aligned}
$$

\section{Experimental analysis}

\subsection{A hypothetical MCS problem}

In this section, a hypothetical MCS problem will be presented, followed by the application of the proposed approach. The MCS problem aims to classify 200 alternatives $a_{1}-a_{200}$ into three predefined and preferentially ordered categories $C_{1}, C_{2}$, and $C_{3}$, where $C_{1}$ and $C_{3}$ consist of the worst and best alternatives, respectively. From the set of alternatives, the first half of alternatives $A^{R}=\left\{a_{1}, \ldots, a_{100}\right\}$ are the reference ones and the other half of alternatives $A=\left\{a_{101}, \ldots, a_{200}\right\}$ are the non-reference ones. The alternatives are evaluated on two criteria $g_{1}$ and $g_{2}$, and the evaluation scale on each criterion $g_{j}, j=1,2$, is bounded in $[0,1]$. The evaluations of the alternatives on the considered criteria can be found in Appendix A.

In order to construct an imbalanced set of reference alternatives, we assume that the actual preference model of the $\mathrm{DM}$ is a linear additive value function $U(a)=0.35 g_{1}(a)+0.65 g_{2}(a), a \in A^{R} \cup A$, and the vector of actual category thresholds is $\mathbf{b}=\left(b_{0}=0, b_{1}=0.6876, b_{2}=0.8484, b_{3}=1.0001\right)$. According to the 
actual preference model and the actual category thresholds, we can calculate the value $U\left(a^{*}\right)$ of each reference alternative $a^{*} \in A^{R}$ and assign $a^{*}$ to categories based on the comparison between $U\left(a^{*}\right)$ and $\mathbf{b}$. Then, we obtain an imbalanced reference set: the category $C_{1}$ containing 70 reference alternatives, the category $C_{2}$ containing 22 reference alternatives, and the category $C_{3}$ containing 8 reference alternatives. Note that we change the actual assignments of five reference alternatives and consider them as outliers: changing the assignment of $a_{4}$ from $C_{3}$ to $C_{2}$, changing the assignment of $a_{27}$ from $C_{1}$ to $C_{3}$, changing the assignment of $a_{39}$ from $C_{1}$ to $C_{3}$, changing the assignment of $a_{51}$ from $C_{1}$ to $C_{3}$, and changing the assignment of $a_{85}$ from $C_{1}$ to $C_{2}$. The assignment examples of the reference alternatives can also be found in Appendix A. The characteristic vectors of the reference alternatives are plotted in Figure 3, in which the characteristic vectors of the reference alternatives of the categories $C_{1}, C_{2}$, and $C_{3}$ are represented by ".", "×", and "*", respectively. From Figure 3 , one can observe that the characteristic vectors of the reference alternatives of different categories are not linearly separable due to the existence of outliers.
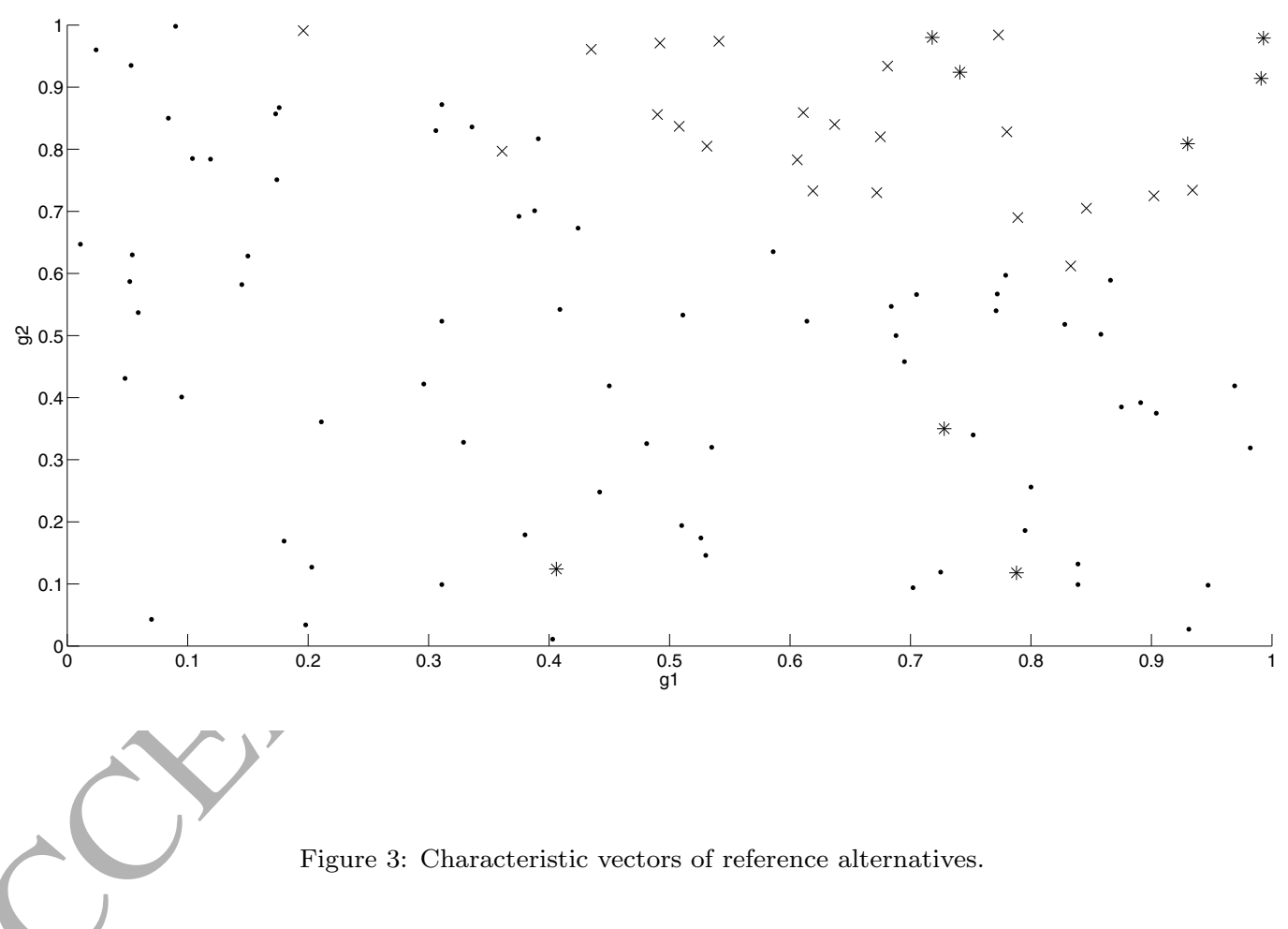

Figure 3: Characteristic vectors of reference alternatives.

We will apply the proposed approach to this MCS problem with an imbalanced set of assignment examples. In Step 1, the hierarchical clustering algorithm is used to group reference alternatives into clusters based on their characteristic vectors. The result of the clustering analysis is reported in Table 2 and depicted in Figure 4, where each cluster of more than one alternative is bounded by dashed lines. It can be observed that the reference alternatives of the categories $C_{1}, C_{2}$, and $C_{3}$ are grouped into six, three, and four clusters, respectively. Particularly, two clusters of the category $C_{2}$ and three clusters of the category $C_{3}$ consist of only one reference alternative for each cluster, which corresponds to a specific outlier.

In Step 2, the proposed approach uses the LP models $L P 5$ and $L P 6$ to identify reference alternatives lying 
Table 2: Result of clustering analysis.

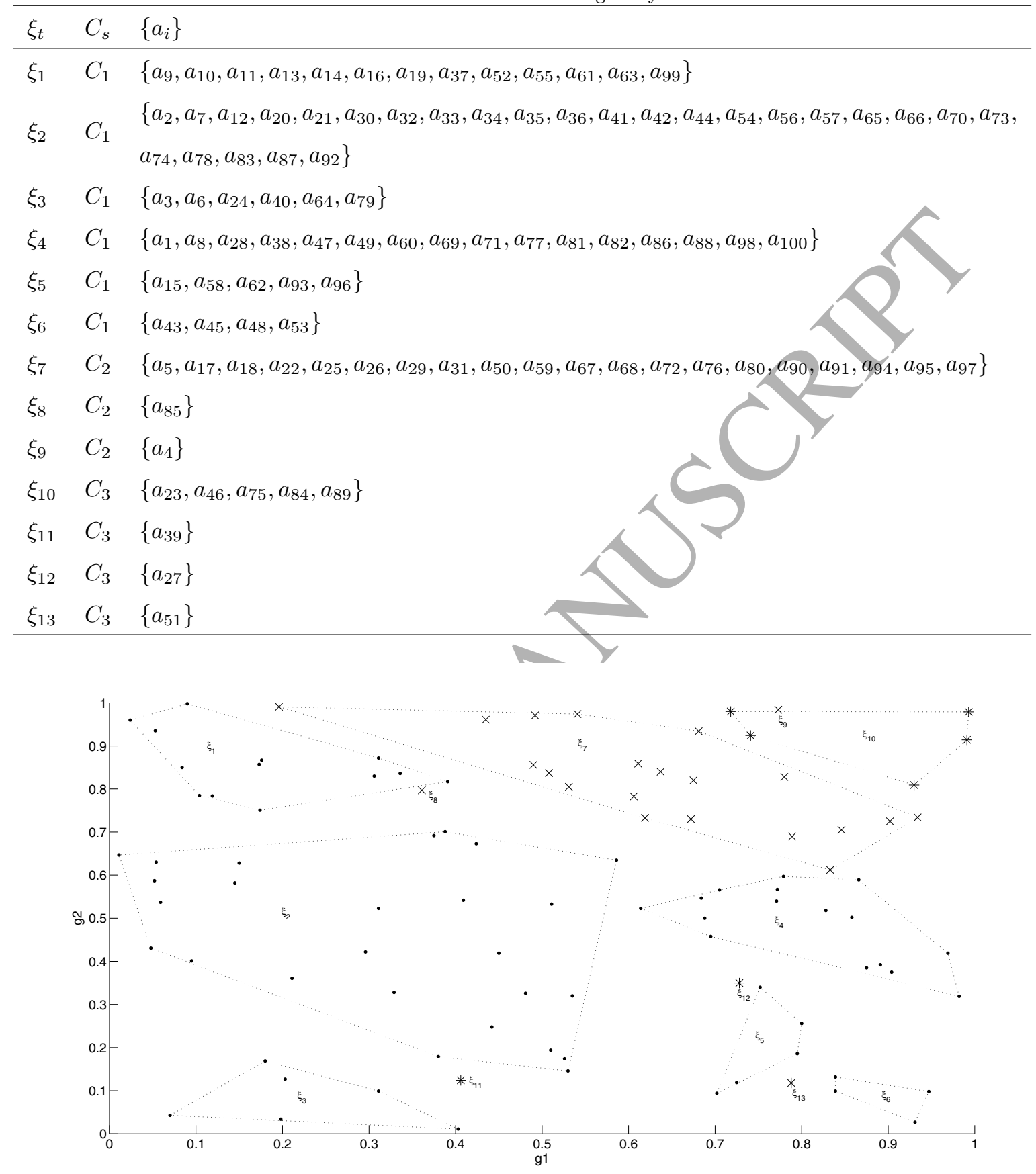

Figure 4: Result of clustering analysis.

in upper and lower preferential boundaries of each cluster. The result is shown in Table 3 and Figure 5, in which the dashed lines represent preferential boundaries of clusters. As we mentioned in Section 2.4, we do not consider the reference alternatives lying in the upper preferential boundaries of clusters in $C_{l}$ and the reference alternatives lying in the lower preferential boundaries of clusters in $C_{1}$. One can observe that the reference 
Table 3: Reference alternatives lying in upper and lower preferential boundaries of each cluster.

\begin{tabular}{llll}
\hline$\xi_{t}$ & $C_{s}$ & $B^{+}\left(\xi_{t}\right)$ & $B^{-}\left(\xi_{t}\right)$ \\
\hline$\xi_{1}$ & $C_{1}$ & $\left\{a_{16}, a_{61}, a_{63}\right\}$ & not considered \\
$\xi_{2}$ & $C_{1}$ & $\left\{a_{7}, a_{30}\right\}$ & not considered \\
$\xi_{3}$ & $C_{1}$ & $\left\{a_{3}, a_{64}, a_{79}\right\}$ & not considered \\
$\xi_{4}$ & $C_{1}$ & $\left\{a_{8}, a_{49}, a_{60}, a_{98}\right\}$ & not considered \\
$\xi_{5}$ & $C_{1}$ & $\left\{a_{58}, a_{96}\right\}$ & not considered \\
$\xi_{6}$ & $C_{1}$ & $\left\{a_{43}, a_{45}\right\}$ & not considered \\
$\xi_{7}$ & $C_{2}$ & $\left\{a_{5}, a_{18}, a_{90}, a_{97}\right\}$ & $\left\{a_{5}, a_{31}, a_{67}\right\}$ \\
$\xi_{8}$ & $C_{2}$ & $\left\{a_{85}\right\}$ & $\left\{a_{85}\right\}$ \\
$\xi_{9}$ & $C_{2}$ & $\left\{a_{4}\right\}$ & $\left\{a_{4}\right\}$ \\
$\xi_{10}$ & $C_{3}$ & not considered & $\left\{a_{23}, a_{75}, a_{89}\right\}$ \\
$\xi_{11}$ & $C_{3}$ & not considered & $\left\{a_{39}\right\}$ \\
$\xi_{12}$ & $C_{3}$ & not considered & $\left\{a_{27}\right\}$ \\
$\xi_{13}$ & $C_{3}$ & not considered & $\left\{a_{51}\right\}$ \\
\hline & & &
\end{tabular}

alternatives lying in upper preferential boundaries of clusters are located at the top-right of the figure while those lying in lower preferential boundaries of clusters are located at the bottom-left.

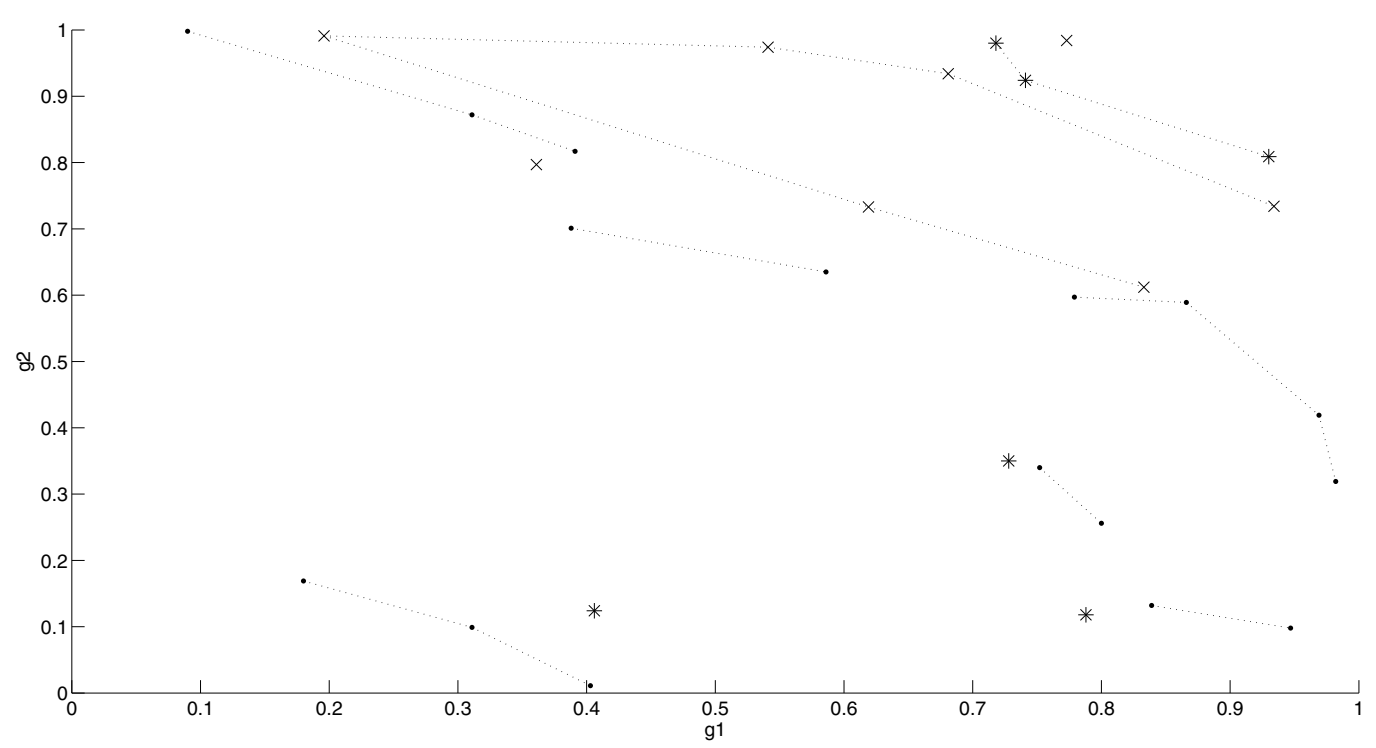

Figure 5: Reference alternatives lying in upper and lower preferential boundaries of each cluster.

In Step 3, the approach uses the developed balancing algorithm to add artificial reference alternatives to the set of reference alternatives lying in preferential boundaries of clusters in each category in order to obtain 
Table 4: Added artificial reference alternatives by the balancing algorithm.

\begin{tabular}{llll}
\hline$\xi_{t}$ & $C_{s}$ & Reference alternatives added to $B^{+}\left(\xi_{t}\right)$ & Reference alternatives added to $B^{-}\left(\xi_{t}\right)$ \\
\hline & & $(0.282,0.987),(0.369,0.983),(0.455,0.978)$, & $(0.408,0.862),(0.726,0.673),(0.303,0.927)$, \\
$\xi_{7}$ & $C_{2}$ & $(0.611,0.954),(0.576,0.964),(0.744,0.884)$, & $(0.513,0.798),(0.249,0.959),(0.355,0.894)$, \\
& & $(0.808,0.834),(0.871,0.784),(0.412,0.980)$, & $(0.460,0.830),(0.566,0.765),(0.673,0.703)$, \\
& $(0.498,0.976)$ & $(0.780,0.642),(0.329,0.910)$ \\
& & & $(0.730,0.952),(0.753,0.917),(0.765,0.910)$, \\
$\xi_{10}$ & $C_{3}$ & & $(0.788,0.895),(0.812,0.881),(0.836,0,867)$, \\
& & & $(0.859,0.852),(0.883,0.838),(0.906,0.823)$, \\
& & &
\end{tabular}

a balanced set of assignment examples. As the number of reference alternatives lying in upper preferential boundaries of clusters in $C_{1}$ is the maximum, i.e., $N=16$, the goal of the balancing algorithm is to adjust the numbers of reference alternatives lying in preferential boundaries of clusters in other categories to be $N$. The outcome of the balancing algorithm is presented in Table 4 and shown in Figure 6. It can be observed that the added artificial reference alternatives are located at the preferential boundaries of clusters.
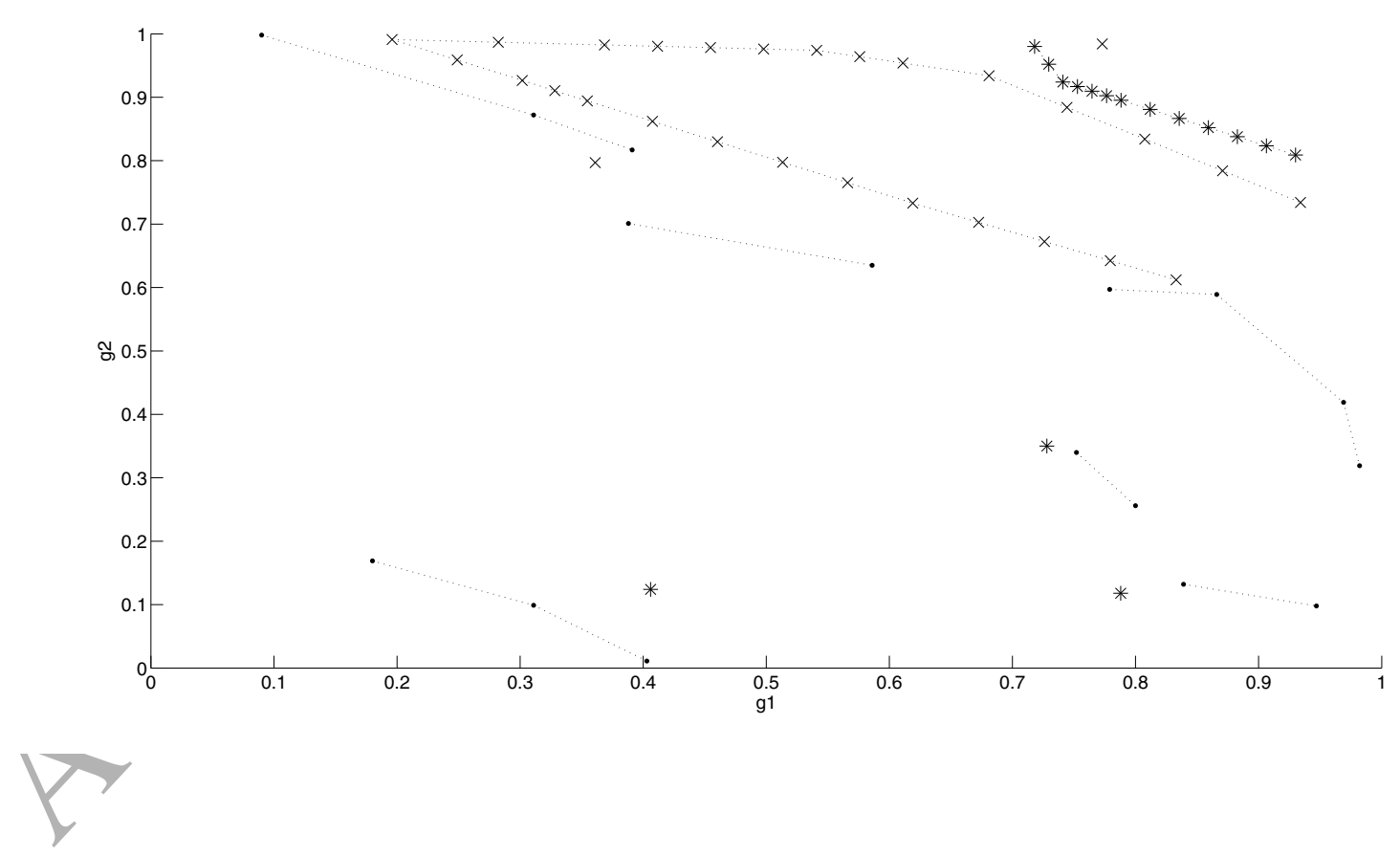

Figure 6: Adjusted reference alternatives lying in upper and lower preferential boundaries of each cluster.

Finally, the proposed approach infers a sorting model by minimizing the sum of violations between the values of reference alternatives lying in preferential boundaries of clusters and the corresponding category thresholds, and then applys the inferred sorting model to assign non-reference alternatives into categories. The inferred value function is $U(a)=0.3451 g_{1}(a)+0.6549 g_{2}(a), a \in A^{R} \cup A$, and the inferred category thresholds 
is $\mathbf{b}=\left(b_{0}=0, b_{1}=0.6847, b_{2}=0.8507, b_{3}=1.0001\right)$. For non-reference alternatives, the actual assignments and the assignments obtained by applying the inferred sorting model are listed in Table 5. Moreover, in order to validate the proposed approach, we also apply the UTADIS method to infer a sorting model. The inferred value function from UTADIS is $U(a)=0.4415 g_{1}(a)+0.5585 g_{2}(a), a \in A^{R} \cup A$, and the inferred category thresholds is $\mathbf{b}=\left(b_{0}=0, b_{1}=0.6619, b_{2}=0.8224, b_{3}=1.0001\right)$. Actually, the UTADIS method identifies eight assignment examples $a_{4} \rightarrow C_{2}, a_{5} \rightarrow C_{2}, a_{27} \rightarrow C_{3}, a_{39} \rightarrow C_{3}, a_{49} \rightarrow C_{1}, a_{51} \rightarrow C_{3}, a_{85} \rightarrow C_{2}, a_{98} \rightarrow C_{1}$ as the ones causing the inconsistency. We note that as $C_{3}$ is the smaller category that contains eight reference alternatives, three assignment examples are identified as the inconsistent ones, which means a low classification accuracy. Then, we apply these sorting models to the non-reference alternatives to obtain assignments that are also reported in Table 5. We observe that for the proposed approach, the assignment of $a_{125}$ is different from the actual assignment and the assignments of the rest non-reference alternatives remain the same as the actual assignment. However, for the UTADIS method, there are six non-reference alternatives, i.e., $a_{105}, a_{122}$, $a_{171}, a_{177}, a_{182}$, and $a_{196}$, whose assignments are different from their actual assignments.

Table 5: Sorting results of different approaches.

\begin{tabular}{|c|c|c|c|c|c|c|c|}
\hline$a_{i}$ & $\begin{array}{l}\text { Actual } \\
\text { assignment }\end{array}$ & $\begin{array}{l}\text { Assignment of } \\
\text { the proposed } \\
\text { approach }\end{array}$ & $\begin{array}{l}\text { Assignment } \\
\text { of UTADIS }\end{array}$ & & $\begin{array}{l}\text { Actual } \\
\text { assignment }\end{array}$ & $\begin{array}{l}\text { Assignment of } \\
\text { the proposed } \\
\text { approach }\end{array}$ & $\begin{array}{l}\text { Assignment } \\
\text { of UTADIS }\end{array}$ \\
\hline$a_{101}$ & $C_{1}$ & $C_{1}$ & 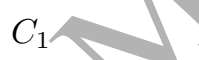 & $a_{151}$ & $C_{1}$ & $C_{1}$ & $C_{1}$ \\
\hline$a_{102}$ & $C_{1}$ & $C_{1}$ & & $a_{152}$ & $C_{3}$ & $C_{3}$ & $C_{3}$ \\
\hline$a_{103}$ & $C_{3}$ & $C_{3}$ & & $a_{153}$ & $C_{1}$ & $C_{1}$ & $C_{1}$ \\
\hline$a_{104}$ & $C_{2}$ & $C_{2}$ & & $a_{154}$ & $C_{1}$ & $C_{1}$ & $C_{1}$ \\
\hline$a_{105}$ & $C_{2}$ & & $C_{3}$ & $a_{155}$ & $C_{1}$ & $C_{1}$ & $C_{1}$ \\
\hline$a_{106}$ & $C_{1}$ & & $C_{1}$ & $a_{156}$ & $C_{1}$ & $C_{1}$ & $C_{1}$ \\
\hline$a_{107}$ & $C_{1}$ & & $C_{1}$ & $a_{157}$ & $C_{2}$ & $C_{2}$ & $C_{2}$ \\
\hline$a_{108}$ & $C_{1}$ & & $C_{1}$ & $a_{158}$ & $C_{1}$ & $C_{1}$ & $C_{1}$ \\
\hline$a_{109}$ & $C_{1}$ & $U_{1}$ & $C_{1}$ & $a_{159}$ & $C_{1}$ & $C_{1}$ & $C_{1}$ \\
\hline$a_{110}$ & 1 & $C_{1}$ & $C_{1}$ & $a_{160}$ & $C_{1}$ & $C_{1}$ & $C_{1}$ \\
\hline$a_{1}$ & $\gamma_{1}$ & $C_{1}$ & $C_{1}$ & $a_{161}$ & $C_{1}$ & $C_{1}$ & $C_{1}$ \\
\hline$a_{112}$ & $C_{1}$ & $C_{1}$ & $C_{1}$ & $a_{162}$ & $C_{1}$ & $C_{1}$ & $C_{1}$ \\
\hline$a_{113}$ & $C_{1}$ & $C_{1}$ & $C_{1}$ & $a_{163}$ & $C_{1}$ & $C_{1}$ & $C_{1}$ \\
\hline$a_{114}$ & $C_{1}$ & $C_{1}$ & $C_{1}$ & $a_{164}$ & $C_{1}$ & $C_{1}$ & $C_{1}$ \\
\hline$a_{115}$ & $C_{1}$ & $C_{1}$ & $C_{1}$ & $a_{165}$ & $C_{1}$ & $C_{1}$ & $C_{1}$ \\
\hline$a_{116}$ & $C_{1}$ & $C_{1}$ & $C_{1}$ & $a_{166}$ & $C_{1}$ & $C_{1}$ & $C_{1}$ \\
\hline$a_{117}$ & $C_{2}$ & $C_{2}$ & $C_{2}$ & $a_{167}$ & $C_{1}$ & $C_{1}$ & $C_{1}$ \\
\hline$a_{118}$ & $C_{2}$ & $C_{2}$ & $C_{2}$ & $a_{168}$ & $C_{1}$ & $C_{1}$ & $C_{1}$ \\
\hline
\end{tabular}




\begin{tabular}{|c|c|c|c|c|c|c|c|}
\hline$a_{119}$ & $C_{1}$ & $C_{1}$ & $C_{1}$ & $a_{169}$ & $C_{3}$ & $C_{3}$ & $C_{3}$ \\
\hline$a_{120}$ & $C_{1}$ & $C_{1}$ & $C_{1}$ & $a_{170}$ & $C_{1}$ & $C_{1}$ & $C_{1}$ \\
\hline$a_{121}$ & $C_{1}$ & $C_{1}$ & $C_{1}$ & $a_{171}$ & $C_{2}$ & $C_{2}$ & $C_{3}$ \\
\hline$a_{122}$ & $C_{1}$ & $C_{1}$ & $C_{2}$ & $a_{172}$ & $C_{1}$ & $C_{1}$ & $C_{1}$ \\
\hline$a_{123}$ & $C_{1}$ & $C_{1}$ & $C_{1}$ & $a_{173}$ & $C_{2}$ & $C_{2}$ & $C_{2}$ \\
\hline$a_{124}$ & $C_{1}$ & $C_{1}$ & $C_{1}$ & $a_{174}$ & $C_{1}$ & $C_{1}$ & $C_{1}$ \\
\hline$a_{125}$ & $C_{3}$ & $C_{2}$ & $C_{3}$ & $a_{175}$ & $C_{1}$ & $C_{1}$ & $C_{1}$ \\
\hline$a_{126}$ & $C_{1}$ & $C_{1}$ & $C_{1}$ & $a_{176}$ & $C_{2}$ & $C_{2}$ & $C_{2}$ \\
\hline$a_{127}$ & $C_{1}$ & $C_{1}$ & $C_{1}$ & $a_{177}$ & $C_{1}$ & $C_{1}$ & $C_{2}$ \\
\hline$a_{128}$ & $C_{3}$ & $C_{3}$ & $C_{3}$ & $a_{178}$ & $C_{1}$ & 1 & $C_{1}$ \\
\hline$a_{129}$ & $C_{1}$ & $C_{1}$ & $C_{1}$ & $a_{179}$ & $C_{1}$ & & $C_{1}$ \\
\hline$a_{130}$ & $C_{1}$ & $C_{1}$ & $C_{1}$ & $a_{180}$ & $C_{1}$ & $C_{1}$ & $C_{1}$ \\
\hline$a_{131}$ & $C_{1}$ & $C_{1}$ & $C_{1}$ & $a_{181}$ & $\gamma_{1}$ & $C_{1}$ & $C_{1}$ \\
\hline$a_{132}$ & $C_{1}$ & $C_{1}$ & $C_{1}$ & $a_{182}$ & & $C_{1}$ & $C_{2}$ \\
\hline$a_{133}$ & $C_{1}$ & $C_{1}$ & $C_{1}$ & 18 & & $C_{1}$ & $C_{1}$ \\
\hline$a_{134}$ & $C_{1}$ & $C_{1}$ & $C_{1}$ & & $C_{1}$ & $C_{1}$ & $C_{1}$ \\
\hline$a_{135}$ & $C_{1}$ & $C_{1}$ & $C_{1}$ & & $C_{1}$ & $C_{1}$ & $C_{1}$ \\
\hline$a_{136}$ & $C_{1}$ & $C_{1}$ & $C_{1}$ & $a_{1 \varepsilon}$ & $C_{2}$ & $C_{2}$ & $C_{2}$ \\
\hline$a_{137}$ & $C_{2}$ & $C_{2}$ & 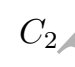 & $a_{187}$ & $C_{1}$ & $C_{1}$ & $C_{1}$ \\
\hline$a_{138}$ & $C_{1}$ & $C_{1}$ & $C$ & $a_{188}$ & $C_{1}$ & $C_{1}$ & $C_{1}$ \\
\hline$a_{139}$ & $C_{1}$ & $C_{1}$ & & $a_{189}$ & $C_{1}$ & $C_{1}$ & $C_{1}$ \\
\hline$a_{140}$ & $C_{1}$ & $C_{1}$ & & $a_{190}$ & $C_{2}$ & $C_{2}$ & $C_{2}$ \\
\hline$a_{141}$ & $C_{3}$ & $C$ & $C_{3}$ & $a_{191}$ & $C_{1}$ & $C_{1}$ & $C_{1}$ \\
\hline$a_{142}$ & $C_{1}$ & & $C_{1}$ & $a_{192}$ & $C_{1}$ & $C_{1}$ & $C_{1}$ \\
\hline$a_{143}$ & $C_{1}$ & & $C_{1}$ & $a_{193}$ & $C_{1}$ & $C_{1}$ & $C_{1}$ \\
\hline$a_{144}$ & $C_{1}$ & & $C_{1}$ & $a_{194}$ & $C_{1}$ & $C_{1}$ & $C_{1}$ \\
\hline$a_{145}$ & $C$ & & $C_{1}$ & $a_{195}$ & $C_{1}$ & $C_{1}$ & $C_{1}$ \\
\hline$a_{14}$ & & $C_{1}$ & $C_{1}$ & $a_{196}$ & $C_{2}$ & $C_{2}$ & $C_{3}$ \\
\hline$a_{147}$ & $C_{1}$ & $C_{1}$ & $C_{1}$ & $a_{197}$ & $C_{1}$ & $C_{1}$ & $C_{1}$ \\
\hline$a_{148}$ & $C_{1}$ & $C_{1}$ & $C_{1}$ & $a_{198}$ & $C_{1}$ & $C_{1}$ & $C_{1}$ \\
\hline$a_{149}$ & $C_{1}$ & $C_{1}$ & $C_{1}$ & $a_{199}$ & $C_{2}$ & $C_{2}$ & $C_{2}$ \\
\hline$a_{150}$ & $C_{1}$ & $C_{1}$ & $C_{1}$ & $a_{200}$ & $C_{2}$ & $C_{2}$ & $C_{2}$ \\
\hline
\end{tabular}

Furthermore, we depict the sorting models inferred from different approaches in Figure 7, in which each sorting model is represented by two parallel lines $U(a)=b_{1}$ and $U(a)=b_{2}$. One can observe that the sorting model inferred from the proposed approach is very close to the actual sorting model. On the other hand, the sorting model inferred from the UTADIS method is far way from the actual sorting model due to the difference 
between the inferred criteria weights and the actual ones.

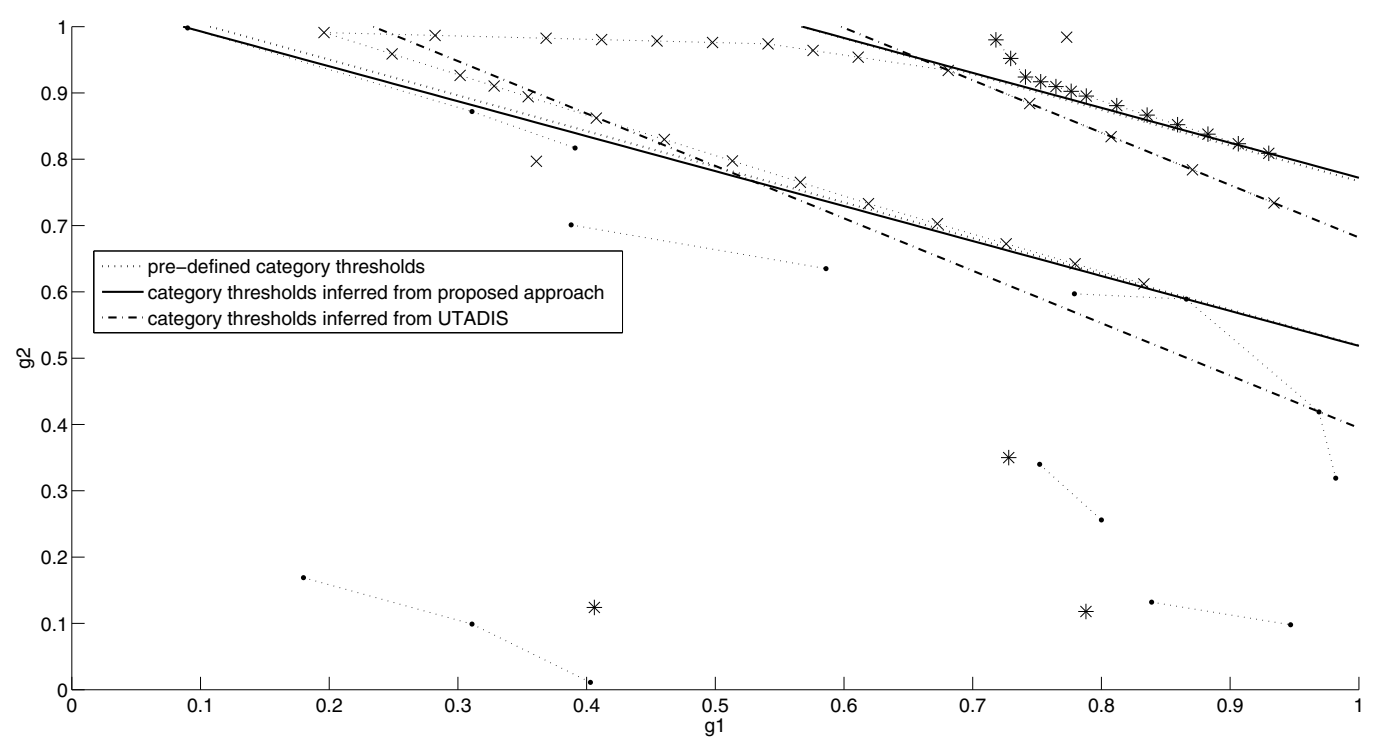

Figure 7: Actual sorting model, sorting model inferred from the proposed approach, sorting model inferred from UTADIS.

\subsection{Test on real data sets}

In this section, we present the results of an experimental study that was conducted in order to validate the practical performance of the proposed approach. To this end, we compare it with the UTADIS method on a number of benchmark data sets.

\subsubsection{Data description}

The data sets are selected from the UCI repository ${ }^{1}$ and the WEKA machine learning datasets ${ }^{2}$. For these data sets, the assumption of monotonicity can be made on the input variables and the output variable. Thus, the input and output variables can be deemed as criteria and category, respectively. Note that, in the phase of preprocessing, the input variables of non-monotonicity (eg. name, gender and so on) are removed, and numerical output variables are categorized by setting thresholds, and incomplete instances are removed. The description of these data sets and their properties is summarized in Table 6 . One can observe that out of the nine data sets, six data sets have imbalanced distributions of alternatives in categories except DBS, CPU, and MMG.

\footnotetext{
${ }^{1}$ http://archive.ics.uci.edu/ml/

${ }^{2}$ http://www.cs.waikato.ac.nz/ml/weka/datasets.html
} 
Table 6: Data sets and their properties.

\begin{tabular}{lllll}
\hline Data set & \#Alternatives & \#Criteria & \#Categories & Distribution \\
\hline Den Bosch (DBS) & 120 & 8 & 2 & $60 / 60$ \\
CPU & 209 & 6 & 4 & $50 / 53 / 53 / 53$ \\
Breast Cancer (BCC) & 278 & 7 & 2 & $196 / 82$ \\
Auto MPG (MPG) & 392 & 7 & 4 & $107 / 154 / 100 / 31$ \\
Employee Selection (ESL) & 488 & 4 & 9 & $2 / 12 / 38 / 100 / 116 /$ \\
& & 5 & $135 / 62 / 19 / 4$ \\
Mammographic (MMG) & 830 & 4 & $427 / 403$ \\
Employee Rejection/Acceptance (ERA) & 1000 & 4 & $415 / 330 / 206 / 49$ \\
Lecturers Evaluation (LEV) & 1000 & 6 & $53 / 280 / 403 / 197 / 27$ \\
Car Evaluation (CEV) & 1728 & 4 & $1210 / 384 / 69 / 65$ \\
\hline
\end{tabular}

In our experiment, we use one half of alternatives in each data set as the reference set and the rest as the test set. Moreover, the distribution of reference alternatives in each reference set is set to be the same with that of test alternatives in the corresponding test set. We infer sorting models using the proposed approach and UTADIS from each reference set. Then, the inferred sorting models are used to obtain assignments for alternatives in the corresponding test set. We repeat this procedure for 100 random splits of each data set. Note that, for the convenience of the following analysis, the number of sub-intervals on each cardinal criterion is set to be equal in our experimental setting while for each ordinal criterion, the evaluations of alternatives are considered as the characteristic points.

In case of $l$ categories, the confusion matrix regarding the MCS problem can be presented in Table 7 where the number of alternatives that are actually assigned to category $C_{i}$ and predicted to be assigned to category $C_{j}$ by the sorting method is denoted by $m_{i j}, 1 \leq i, j \leq l$. We consider the following measures to evaluate the performances of the sorting methods. The first measure is classification accuracy, which is defined as

$$
1 \text { Accuracy }=\frac{\sum_{i=1}^{l} m_{i i}}{\sum_{i, j=1}^{l} m_{i j}} \text {. }
$$

This metric provides a simple way of describing the performance of a sorting method on a given data set. However, it is inadequate in reflecting the performance on each single category, especially on those small categoriés. In lieu of accuracy, other evaluation measures are frequently adopted in the literature to provide comprehensive assessments of imbalanced learning problems, namely, Precision, Recall, F- measure, and $G-\operatorname{mean}[47,48]$, as follows:

$$
\begin{gathered}
\text { Recall }_{i}=\frac{m_{i i}}{\sum_{j=1}^{l} m_{i j}}, \\
\text { Precision }_{i}=\frac{m_{i i}}{\sum_{j=1}^{l} m_{j i}},
\end{gathered}
$$




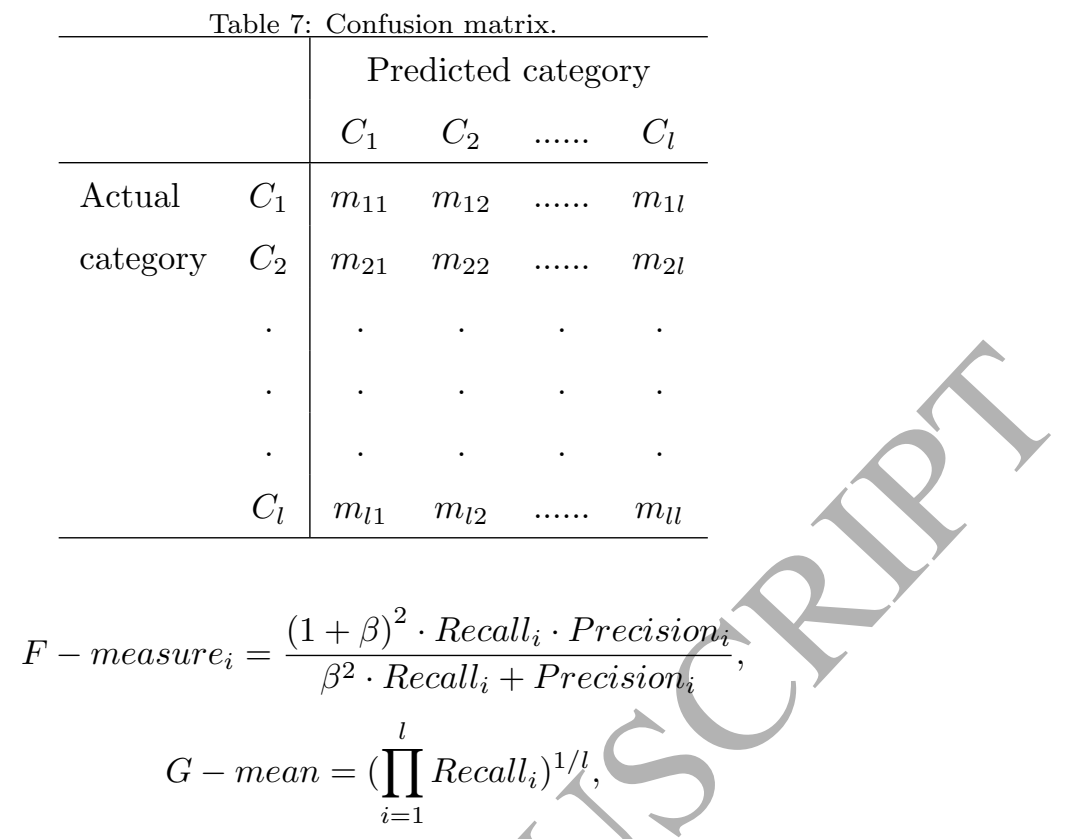

where $\beta$ is a coefficient to adjust the relative importance of recall versus precision (usually, $\beta=1$ ). Intuitively, Recall $_{i}$ is a measure of completeness (i.e., how many alternatives that are actually assigned to category $C_{i}$ are correctly assigned by the sorting method), whereas Precision $_{i}$ is a measure of exactness (i.e., of the alternatives that are assigned to category $C_{i}$ by the sorting method, how many are actually assigned to category $\left.C_{i}\right)$. Recall $l_{i}$ and Precision $_{i}$ share an inverse relationship between each other. Thus, the $F-$ measure $_{i}$ metric combines precision and recall as a measure of the performance of the sorting method. It is obvious that if the $F$-measure $e_{i}$ metric is high when both Recall $_{i}$ and Precision $_{i}$ should be high. The $G$ - mean metric accounts for each Recall $_{i}$ equally and thus is capable of measuring the balanced performance among all the categories.

The platform for conducting the experimental analysis is a Linux PC with 4.2GHz CPU and 16GB RAM. We solved all optimization problems (linear and mixed-integer) using CPLEX 12.7 and implement all algorithms with Java language.

\subsubsection{Experiment results}

Appendix B reports the classification results (mean values of Accuracy, $F$ - measure, and $G$-mean) obtained with the proposed approach and UTADIS and the results of $t$-test that is used to check whether any paired classification results are significantly different from each other with $95 \%$ confidence. Accuracy and $G-$ mean measure the overall classification performances of MCS methods on all the categories, whereas $F-$ measure $_{i}$ assesses the classification performance on each individual category. One can observe that, on one hand, the proposed approach and UTADIS attain similar classification performances on balanced datasets (i.e., DBS, CPU, and MMG), including the overall classification performances on all the categories and those on each individual category. The result of $t$-test for most Accuracy and $G$ - mean on DBS, CPU, and MMG is not significant. On the other hand, the proposed approach outperforms UTADIS on those imbalanced data sets (i.e., BCC, MPG, ESL, ERA, LEV, and CEV). Particularly, the proposed approach obtains higher 
Table 8: Average of number of misclassified test alternatives whose characteristic vectors are located at convex hulls of clusters.

\begin{tabular}{lrrrr}
\multirow{2}{*}{ Data set } & \multicolumn{3}{c}{ The proposed approach } & \multicolumn{3}{c}{ UTADIS } \\
& Reference alternatives & Test alternatives & Reference alternatives & Test alternatives \\
\hline DBS & 8.36 & 7.83 & 5.89 & 6.32 \\
CPU & 8.31 & 9.47 & 10.24 & 11.58 \\
BCC & 27.63 & 30.92 & 48.65 & 43.85 \\
MPG & 40.91 & 48.53 & 55.39 & 53.87 \\
ESL & 22.92 & 25.35 & 39.20 & 42.76 \\
MMG & 46.13 & 54.80 & 68.81 & 79.11 \\
ERA & 30.41 & 32.85 & 91.17 & 102.13 \\
LEV & 67.08 & 84.33 & 127.33 & 134.14 \\
CEV & 42.45 & 61.41 & 109.15 & 114.45 \\
\hline
\end{tabular}

$F$-measure on small categories than UTADIS, which shows that the performance of the proposed approach on small categories is improved than UTADIS. The result of $t$-test for most Accuracy, $G$-mean, and $F$-measure $i$ is significant.

To investigate why the proposed approach outperforms UTADIS, we report the average of number of misclassified alternatives (including reference ones and est ones) whose characteristic vectors are located at convex hulls of clusters in each test in Table 8. As proved by Proposition 4, if reference alternatives lying in preferential boundaries of a cluster are correctly classified, then all the others in this cluster are also correctly classified. Thus, we believe that more alternatives whose characteristic vectors are located at convex hulls of clusters can be correctly classified by the proposed approach. Table 8 confirms our expectation and illustrates that less alternatives whose characteristic vectors are located at convex hulls of clusters are misclassified by the proposed approach. In contrast, as UTADIS does not utilize the distribution of characteristic vectors of alternatives, the developed sorting model will be biased towards categories with more reference alternatives, which leads to the misclassification of more alternatives.

Furthermore, to demonstrate the combination of the clustering algorithm with the balancing algorithm, we report the classification results of applying the model $L P 8$ to the results of the clustering algorithm without the balancing algorithm in Appendix C. One can clearly observe that the results obtained without the balancing algorithm are worse than those obtained with the combination of the clustering algorithm with the balancing algorithm, and the difference is statistically significant with $95 \%$ confidence. Particularly, for small categories in imbalanced data sets, the $F$ - measure ${ }_{i}$ metrics of the results without the balancing algorithm deteriorate severely, such as $F$ - measure in $_{4}$ MPG and $F$ - measure ${ }_{1}$ and $F$ - measure ${ }_{9}$ in ESL. This illustrates that the improvement of the proposed approach is due to the combination of the clustering algorithm and the balancing algorithm.

Table 9 summarizes the average computation time (in CPU seconds) and proportion of each phase of the 
Table 9: Average CPU time and portion of each step of the proposed approach for each data set.

\begin{tabular}{|c|c|c|c|c|c|}
\hline & CPU time & $\begin{array}{l}\text { \%Clustering } \\
\text { analysis }\end{array}$ & $\begin{array}{l}\% \text { Finding } B^{+}\left(\xi_{t}\right) \\
\text { and } B^{-}\left(\xi_{t}\right)\end{array}$ & $\begin{array}{l}\text { \% Balancing } \\
\text { algorithm }\end{array}$ & $\begin{array}{l}\text { \%Inferring } \\
\text { sorting model }\end{array}$ \\
\hline DBS & 10.14 & 41.27 & 35.32 & 17.43 & 5.98 \\
\hline $\mathrm{CPU}$ & 62.07 & 46.98 & 34.29 & 14.24 & 4.49 \\
\hline $\mathrm{BCC}$ & 105.79 & 47.24 & 31.87 & 10.32 & 10.57 \\
\hline MPG & 214.87 & 58.29 & 29.76 & 7.43 & \\
\hline ESL & 312.49 & 61.27 & 26.40 & 8.72 & 3.61 \\
\hline MMG & 1009.64 & 68.72 & 17.45 & 6.26 & 7.57 \\
\hline ERA & 1354.66 & 74.91 & 12.31 & & 7.55 \\
\hline $\mathrm{LEV}$ & 1446.14 & 72.87 & 11.43 & & 8.44 \\
\hline $\mathrm{CEV}$ & 4012.81 & 86.38 & 6.39 & 4.20 & 3.03 \\
\hline
\end{tabular}

proposed approach on the data sets. As we discussed in Section 2.7, the proposed approach requires more computation time with the increase of the size of a data set. Most of the total CPU time is spent on grouping reference alternatives into clusters. Particularly, the portion of computation time for the clustering analysis increases when a data set contains more alternatives, from $41.27 \%$ on DBS to $86.38 \%$ on CEV.

\section{Conclusions}

In this paper, we presented a new approach for the MCS problem with an imbalanced set of reference alternatives. The approach employs a piecewise-linear additive value function as the preference model and adopts the disaggregation-aggregation paradigm to infer a sorting model from the assignment examples provided by the DM. It employs a hierarchical clustering algorithm and several linear programming models to identify reference alternatives that are active to develop the sorting model and proposes a balancing algorithm to balance active reference alternatives across categories, so that a balanced set of assignment examples can be obtained. The sorting model is obtained by minimizing the sum of violations between values of active reference alternatives and corresponding category thresholds. The experimental study has shown that the approach can achieve improved sorting results in comparison with the UTADIS method especially when a set of reference alternatives reveal severe imbalance.

We envisage further research towards several different directions. Firstly, we hope to extend this study to other forms of preference models including outranking relations and rule-based models. Then, we will compare the approach to cost-sensitive techniques that incorporates a misclassification cost matrix into classical MCS methods in terms of the classification accuracy and the robustness concern. Moreover, considering that how to set $\gamma_{j}$ is an important concern to apply the proposed approach, we intend to propose several selection criteria that combine the accuracy measure and the model complexity, and perform a resampling analysis (e.g., cross-validation) to choose the optimal $\gamma_{j}$. Finally, as one can observe from the results of experimental study, 
it becomes computationally difficult to group reference alternatives into clusters when the number of reference alternatives increases. One possible way to address such a problem is to utilize the MapReduce framework [49] to divide a large reference set into splits and use the clustering algorithm to group reference alternatives into clusters in parallel. Then, a collective sorting model can be developed by aggregating the clustering results from each split in a consistent way.

\section{Acknowledgement}

The research is supported by the National Natural Science Foundation of China \#91546119). We are grateful to the anonymous reviewers for their constructive and detailed comments, which helped us improve the previous version of the paper.

\section{References}

\section{References}

[1] S. Greco, V. Mousseau, R. Słowiński, Multiple criteria sorting with a set of additive value functions, European Journal of Operational Research 207 (3) (2010) 1455-1470.

[2] M. Doumpos, C. Zopounidis, Developing sorting models using preference disaggregation analysis: An experimental investigation, European Journal of Operational Research 154 (3) (2004) 585-598.

[3] S. Corrente, S. Greco, M. Kadziński, R. Słowiński, Robust ordinal regression in preference learning and ranking, Machine Learning 93 (2-3) (2013) 381-422.

[4] M. Kadziński, S. Corrente, S. Greco, R. Słowiński, Preferential reducts and constructs in robust multiple criteria ranking and sorting, OR Spectrum 36 (4) (2014) 1021-1053.

[5] M. Kadziński, K. Ciomek, P. Rychły, R. Słowiński, Post factum analysis for robust multiple criteria ranking and sorting, Journal of Global Optimization (2016) 1-32.

[6] M. Doumpos, C. Zopounidis, Multicriteria decision aid classification methods, Vol. 73, Springer Science \& Business Media, 2002.

[7] M. Köksalan, S. B. Özpeynirci, An interactive sorting method for additive utility functions, Computers \& Operations Research 36 (9) (2009) 2565-2572.

[8] C. Ulu, M. Köksalan, An interactive approach to multicriteria sorting for quasiconcave value functions, Naval Research Logistics (NRL) 61 (6) (2014) 447-457.

[9] M. Kadziński, K. Ciomek, R. Słowiński, Modeling assignment-based pairwise comparisons within integrated framework for value-driven multiple criteria sorting, European Journal of Operational Research 241 (3) (2015) 830-841. 
[10] C. Zopounidis, M. Doumpos, Building additive utilities for multi-group hierarchical discrimination: the mh dis method*, Optimization Methods and Software 14 (3) (2000) 219-240.

[11] O. Cailloux, P. Meyer, V. Mousseau, Eliciting electre tri category limits for a group of decision makers, European Journal of Operational Research 223 (1) (2012) 133-140.

[12] J. Zheng, S. A. M. Takougang, V. Mousseau, M. Pirlot, Learning criteria weights of an optimistic electre tri sorting rule, Computers \& Operations Research 49 (2014) 28-40.

[13] J. Almeida-Dias, J. R. Figueira, B. Roy, Electre tri-c: A multiple criteria sorting method based on characteristic reference actions, European Journal of Operational Research 204 (3) (2010) 565-580.

[14] J. Almeida-Dias, J. R. Figueira, B. Roy, A multiple criteria sorting method where each category is characterized by several reference actions: The electre tri-nc method, European Journal of Operational Research 217 (3) (2012) 567-579.

[15] M. Kadziński, T. Tervonen, J. R. Figueira, Robust multi-criteria sorting with the outranking preference model and characteristic profiles, Omega 55 (2015) 126-140.

[16] P. Nemery, C. Lamboray, Flowsort: a flow-based sorting method with limiting or central profiles, top 16: 90-113 (2008).

[17] Y. Chen, K. W. Hipel, D. M. Kilgour, Multiple-criteria sorting using case-based distance models with an application in water resources management, IEEE Transactions on Systems, Man, and Cybernetics-Part A: Systems and Humans 37 (5) (2007) 680-691.

[18] Y. Chen, K. W. Li, D. M. Kilgour, K. W. Hipel, A case-based distance model for multiple criteria abc analysis, Computers \& Operations Research 35 (3) (2008) 776-796.

[19] S. Greco, B. Matarazzo, R. Slowinski, Rough sets theory for multicriteria decision analysis, European journal of operational research 129 (1) (2001) 1-47.

[20] K. Dembczyński, S. Greco, R. Słowiński, Rough set approach to multiple criteria classification with imprecise evaluations and assignments, European Journal of Operational Research 198 (2) (2009) 626-636.

[21] S. Chakhar, I. Saad, Dominance-based rough set approach for groups in multicriteria classification problems, Decision Support Systems 54 (1) (2012) 372-380.

[22] S. Greco, R. Słowiński, J. R. Figueira, V. Mousseau, Robust ordinal regression, in: M. Ehrgott, J. R. Figueira, S. Greco (Eds.), Trends in Multiple Criteria Decision Analysis, Springer US, 2010, pp. $241-283$.

[23] S. Greco, M. Kadziński, V. Mousseau, R. Słowiński, Robust ordinal regression for multiple criteria group decision: Uta gms-group and utadis gms-group, Decision Support Systems 52 (3) (2012) 549-561. 
[24] M. Kadziński, S. Greco, R. Słowiński, Selection of a representative value function for robust ordinal regression in group decision making, Group Decision and Negotiation 22 (3) (2013) 429-462.

[25] S. Corrente, M. Doumpos, S. Greco, R. Słowiński, C. Zopounidis, Multiple criteria hierarchy process for sorting problems based on ordinal regression with additive value functions, Annals of Operations Research (2015) 1-23.

[26] M. Kadziński, R. Słowiński, Parametric evaluation of research units with respect to reference profiles, Decision Support Systems 72 (2015) 33-43.

[27] J. Liu, X. Liao, W. Zhao, N. Yang, A classification approach based on the outranking model for multiple criteria abc analysis, Omega 61 (2016) 19-34.

[28] S. Angilella, S. Mazzù, The financing of innovative smes: A multicriteria credit rating model, European Journal of Operational Research 244 (2) (2015) 540-554.

[29] M. Doumpos, C. Zopounidis, A multicriteria outranking modeling approach for credit rating, Decision Sciences 42 (3) (2011) 721-742.

[30] M. F. Norese, V. Carbone, An application of electre tri to support innovation, Journal of Multi-Criteria Decision Analysis 21 (1-2) (2014) 77-93.

[31] F. Abastante, M. Bottero, S. Greco, I. Lami, Addressing the location of undesirable facilities through the dominance-based rough set approach, Journal of Multi-Criteria Decision Analysis 21 (1-2) (2014) 3-23.

[32] D. Mailly, I. Abi-Zeid, S. Pepin, A multi-criteria classification approach for identifying favourable climates for tourism, Journal of Multi-CriteriaDecision Analysis 21 (1-2) (2014) 65-75.

[33] F. Macary, J. Almeida-Dias, D. Uny, A. Probst, Assessment of the effects of best environmental practices on reducing pesticide contamination in surface water, using multi-criteria modelling combined with a gis, International Journal of Multicriteria Decision Making 733 (2-3) (2013) 178-211.

[34] C. M. de Miranda Mota, A. T. de Almeida, A multicriteria decision model for assigning priority classes to activities in project management, Annals of operations research 199 (1) (2012) 361-372.

[35] L. Marin, D. Isern, A. Moreno, A. Valls, On-line dynamic adaptation of fuzzy preferences, Information Sciences $220(2013) 5-21$.

[36] E. Fernandez, J. Navarro, A. Duarte, G. Ibarra, Core: A decision support system for regional competitiveness analysis based on multi-criteria sorting, Decision Support Systems 54 (3) (2013) 1417-1426.

[37] A. Ulucan, K. B. Atici, A multiple criteria sorting methodology with multiple classification criteria and an application to country risk evaluation, Technological and Economic Development of Economy 19 (1) (2013) 93-124. 
[38] S. Greco, B. Matarazzo, R. Slowinski, S. Zanakis, Global investing risk: a case study of knowledge assessment via rough sets, Annals of Operations Research 185 (1) (2011) 105-138.

[39] Y. Marinakis, M. Marinaki, M. Doumpos, N. Matsatsinis, C. Zopounidis, Optimization of nearest neighbor classifiers via metaheuristic algorithms for credit risk assessment, Journal of Global Optimization 42 (2) (2008) 279-293.

[40] D. Niklis, M. Doumpos, C. Zopounidis, Combining market and accounting-based models for credit scoring using a classification scheme based on support vector machines, Applied Mathematics and Computation 234 (2014) 69-81.

[41] M. Doumpos, C. Zopounidis, Regularized estimation for preference disaggregation in multiple criteria decision making, Computational Optimization and Applications 38 (1)(2007) 61-80.

[42] R. L. Keeney, H. Raiffa, Decisions with multiple objectives: preferences and value trade-offs, Cambridge university press, 1993.

[43] S. Greco, V. Mousseau, R. Słowiński, Ordinal regression revisited: multiple criteria ranking using a set of additive value functions, European Journal of Operational Research 191 (2) (2008) 416-436.

[44] D. Bertsimas, R. Shioda, Classification and regressionviaginteger optimization, Operations Research 55 (2) (2007) 252-271.

[45] P. Berkhin, A survey of clustering data mining techniques, in: Grouping multidimensional data, Springer, 2006, pp. 25-71.

[46] D. Bertsimas, J. N. Tsitsiklis, Introduction to linear optimization, Vol. 6, Athena Scientific Belmont, MA, 1997.

[47] H. He, E. A. Gareia, Learning from imbalanced data, IEEE Transactions on knowledge and data engineering 21 (9) (2009) 1263-1284.

[48] Y. Sun, M. S. Kamel, Y. Wang, Boosting for learning multiple classes with imbalanced class distribution, in: Data Mining, 2006. ICDM'06. Sixth International Conference on, IEEE, 2006, pp. 592-602.

[49] J. Dean, S. Ghemawat, Mapreduce: simplified data processing on large clusters, Communications of the ACM 51 (1) (2008) 107-113.

Appendix A. Evaluations of alternatives on considered criteria.

\begin{tabular}{llll|llll}
\hline Reference alternative & $\mathrm{g} 1$ & $\mathrm{~g} 2$ & Assignment examples & Non-reference alternative & $\mathrm{g} 1$ & $\mathrm{~g} 2$ & \\
\hline
\end{tabular}




\begin{tabular}{|c|c|c|c|c|c|c|}
\hline$a_{1}$ & 0.904 & 0.375 & $C_{1}$ & $a_{101}$ & 0.382 & 0.187 \\
\hline$a_{2}$ & 0.059 & 0.537 & $C_{1}$ & $a_{102}$ & 0.119 & 0.191 \\
\hline$a_{3}$ & 0.311 & 0.099 & $C_{1}$ & $a_{103}$ & 0.864 & 0.96 \\
\hline$a_{4}$ & 0.773 & 0.984 & $C_{2}$ & $a_{104}$ & 0.807 & 0.742 \\
\hline$a_{5}$ & 0.196 & 0.991 & $C_{2}$ & $a_{105}$ & 0.824 & 0.825 \\
\hline$a_{6}$ & 0.203 & 0.127 & $C_{1}$ & $a_{106}$ & 0.092 & 0.578 \\
\hline$a_{7}$ & 0.586 & 0.635 & $C_{1}$ & $a_{107}$ & 0.783 & 0.131 \\
\hline$a_{8}$ & 0.982 & 0.319 & $C_{1}$ & $a_{108}$ & 0.37 & 0.646 \\
\hline$a_{9}$ & 0.174 & 0.751 & $C_{1}$ & $a_{109}$ & 0.231 & 0.859 \\
\hline$a_{10}$ & 0.336 & 0.836 & $C_{1}$ & $a_{110}$ & 0.322 & 0.586 \\
\hline$a_{11}$ & 0.176 & 0.867 & $C_{1}$ & $a_{111}$ & 0.137 & 0.055 \\
\hline$a_{12}$ & 0.011 & 0.647 & $C_{1}$ & $u_{1}$ & 0.578 & 0.207 \\
\hline$a_{13}$ & 0.104 & 0.785 & $C_{1}$ & $a_{113}$ & 0.777 & 0.047 \\
\hline$a_{14}$ & 0.024 & 0.96 & $C_{1}$ & & 0.906 & 0.025 \\
\hline$a_{15}$ & 0.702 & 0.094 & $C_{1}$ & $a$ & 0.117 & 0.623 \\
\hline$a_{16}$ & 0.311 & 0.872 & $C_{1}$ & $a_{116}$ & 0.706 & 0.296 \\
\hline$a_{17}$ & 0.49 & 0.856 & $C_{2}$ & $a_{117}$ & 0.347 & 0.95 \\
\hline$a_{18}$ & 0.541 & 0.974 & $C_{2}$ & $a_{118}$ & 0.55 & 0.851 \\
\hline$a_{19}$ & 0.084 & 0.85 & $C_{1}$ & $a_{119}$ & 0.971 & 0.366 \\
\hline$a_{20}$ & 0.048 & 0.431 & & $a_{120}$ & 0.485 & 0.171 \\
\hline$a_{21}$ & 0.53 & & & $a_{121}$ & 0.226 & 0.368 \\
\hline$a_{22}$ & 0.61 & 0.859 & $C_{2}$ & $a_{122}$ & 0.829 & 0.566 \\
\hline$a_{23}$ & 47 & 0.924 & $C_{3}$ & $a_{123}$ & 0.511 & 0.029 \\
\hline$a_{24}$ & & 0.034 & $C_{1}$ & $a_{124}$ & 0.208 & 0.022 \\
\hline$a_{25}$ & & 0.69 & $C_{2}$ & $a_{125}$ & 0.941 & 0.803 \\
\hline$a_{26}$ & & 0.705 & $C_{2}$ & $a_{126}$ & 0.689 & 0.281 \\
\hline$a_{27}$ & 0.728 & 0.35 & $C_{3}$ & $a_{127}$ & 0.078 & 0.194 \\
\hline$a_{28}$ & 0.771 & 0.54 & $C_{1}$ & $a_{128}$ & 0.925 & 0.959 \\
\hline & 0.606 & 0.783 & $C_{2}$ & $a_{129}$ & 0.429 & 0.033 \\
\hline$a_{30}$ & 0.388 & 0.701 & $C_{1}$ & $a_{130}$ & 0.414 & 0.082 \\
\hline$a_{31}$ & 0.833 & 0.612 & $C_{2}$ & $a_{131}$ & 0.272 & 0.159 \\
\hline$a_{32}$ & 0.481 & 0.326 & $C_{1}$ & $a_{132}$ & 0.071 & 0.235 \\
\hline$a_{33}$ & 0.052 & 0.587 & $C_{1}$ & $a_{133}$ & 0.974 & 0.175 \\
\hline$a_{34}$ & 0.442 & 0.248 & $C_{1}$ & $a_{134}$ & 0.586 & 0.62 \\
\hline$a_{35}$ & 0.15 & 0.628 & $C_{1}$ & $a_{135}$ & 0.021 & 0.556 \\
\hline$a_{36}$ & 0.145 & 0.582 & $C_{1}$ & $a_{136}$ & 0.19 & 0.741 \\
\hline
\end{tabular}




\begin{tabular}{|c|c|c|c|c|c|c|}
\hline$a_{37}$ & 0.173 & 0.857 & $C_{1}$ & $a_{137}$ & 0.612 & 0.792 \\
\hline$a_{38}$ & 0.695 & 0.458 & $C_{1}$ & $a_{138}$ & 0.782 & 0.076 \\
\hline$a_{39}$ & 0.406 & 0.124 & $C_{3}$ & $a_{139}$ & 0.809 & 0.439 \\
\hline$a_{40}$ & 0.07 & 0.043 & $C_{1}$ & $a_{140}$ & 0.605 & 0.056 \\
\hline$a_{41}$ & 0.095 & 0.401 & $C_{1}$ & $a_{141}$ & 0.937 & 0.939 \\
\hline$a_{42}$ & 0.38 & 0.179 & $C_{1}$ & $a_{142}$ & 0.93 & 0.107 \\
\hline$a_{43}$ & 0.839 & 0.132 & $C_{1}$ & $a_{143}$ & 0.788 & 0.182 \\
\hline$a_{44}$ & 0.296 & 0.422 & $C_{1}$ & $a_{144}$ & 0.579 & 0.507 \\
\hline$a_{45}$ & 0.947 & 0.098 & $C_{1}$ & $a_{145}$ & 0.2 & 0.131 \\
\hline$a_{46}$ & 0.993 & 0.979 & $C_{3}$ & $a_{146}$ & 0.164 & 0.363 \\
\hline$a_{47}$ & 0.705 & 0.566 & $C_{1}$ & $a_{147}$ & 0.684 & 0.561 \\
\hline$a_{48}$ & 0.839 & 0.099 & $C_{1}$ & & 0.551 & 0.287 \\
\hline$a_{49}$ & 0.779 & 0.597 & $C_{1}$ & & 0.103 & 0.816 \\
\hline$a_{50}$ & 0.902 & 0.725 & $C_{2}$ & & 0.843 & 0.068 \\
\hline$a_{51}$ & 0.788 & 0.118 & $C_{3}$ & & 0.6 & 0.476 \\
\hline$a_{52}$ & 0.306 & 0.83 & $C_{1}$ & $a_{152}$ & 0.97 & 0.814 \\
\hline$a_{53}$ & 0.931 & 0.027 & $C_{1}$ & $a_{153}$ & 0.481 & 0.709 \\
\hline$a_{54}$ & 0.375 & 0.692 & $C_{1}$ & $a_{154}$ & 0.205 & 0.766 \\
\hline$a_{55}$ & 0.053 & 0.935 & $C_{1}$ & $a_{155}$ & 0.552 & 0.059 \\
\hline$a_{56}$ & 0.511 & 0.533 & $C_{1}$ & $a_{156}$ & 0.716 & 0.045 \\
\hline$a_{57}$ & 0.535 & 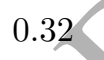 & $e_{1}$ & $a_{157}$ & 0.645 & 0.877 \\
\hline$a_{58}$ & 0.752 & & 1 & $a_{158}$ & 0.69 & 0.026 \\
\hline$a_{59}$ & 0.531 & 0. & $C_{2}$ & $a_{159}$ & 0.065 & 0.235 \\
\hline$a_{60}$ & & 0.419 & $C_{1}$ & $a_{160}$ & 0.398 & 0.057 \\
\hline$a_{61}$ & & 0.998 & $C_{1}$ & $a_{161}$ & 0.46 & 0.77 \\
\hline$a_{62}$ & & 0.186 & $C_{1}$ & $a_{162}$ & 0.557 & 0.736 \\
\hline$a_{63}$ & 0.391 & 0.817 & $C_{1}$ & $a_{163}$ & 0.196 & 0.61 \\
\hline$a_{64}$ & 0.403 & 0.011 & $C_{1}$ & $a_{164}$ & 0.719 & 0.054 \\
\hline$a_{65}$ & 0.311 & 0.523 & $C_{1}$ & $a_{165}$ & 0.666 & 0.182 \\
\hline$a_{66}$ & 0.45 & 0.419 & $C_{1}$ & $a_{166}$ & 0.247 & 0.058 \\
\hline$a_{67}$ & 0.619 & 0.733 & $C_{2}$ & $a_{167}$ & 0.465 & 0.394 \\
\hline$a_{68}$ & 0.637 & 0.84 & $C_{2}$ & $a_{168}$ & 0.368 & 0.058 \\
\hline$a_{69}$ & 0.684 & 0.547 & $C_{1}$ & $a_{169}$ & 0.997 & 0.959 \\
\hline$a_{70}$ & 0.526 & 0.174 & $C_{1}$ & $a_{170}$ & 0.211 & 0.608 \\
\hline$a_{71}$ & 0.614 & 0.523 & $C_{1}$ & $a_{171}$ & 0.737 & 0.897 \\
\hline$a_{72}$ & 0.508 & 0.837 & $C_{2}$ & $a_{172}$ & 0.398 & 0.354 \\
\hline
\end{tabular}




\begin{tabular}{|c|c|c|c|c|c|}
\hline$a_{73}$ & 0.424 & $0.673 \quad C_{1}$ & $a_{173}$ & 0.697 & 0.917 \\
\hline$a_{74}$ & 0.054 & 0.63 & $a_{174}$ & 0.172 & 0.627 \\
\hline$a_{75}$ & 0.93 & $0.809 \quad C_{3}$ & $a_{175}$ & 0.126 & 0.59 \\
\hline$a_{76}$ & 0.672 & 0.73 & $a_{176}$ & 0.67 & 0.86 \\
\hline$a_{77}$ & 0.772 & $0.567 \quad C_{1}$ & $a_{177}$ & 0.901 & 0.547 \\
\hline$a_{78}$ & 0.329 & $0.328 \quad C_{1}$ & $a_{178}$ & 0.212 & 0.151 \\
\hline$a_{79}$ & 0.18 & $0.169 \quad C_{1}$ & $a_{179}$ & 0.692 & 0.477 \\
\hline$a_{80}$ & 0.435 & $0.961 \quad C_{2}$ & $a_{180}$ & 0.275 & 0.434 \\
\hline$a_{81}$ & 0.828 & $0.518 \quad C_{1}$ & $a_{181}$ & 0.91 & 0.244 \\
\hline$a_{82}$ & 0.875 & $0.385 \quad C_{1}$ & $a_{182}$ & 0.809 & 0.584 \\
\hline$a_{83}$ & 0.409 & $0.542 \quad C_{1}$ & $a_{183}$ & 0.287 & 0.769 \\
\hline$a_{84}$ & 0.991 & $0.914 \quad C_{3}$ & & 0.239 & 0.685 \\
\hline$a_{85}$ & 0.361 & $0.797 \quad C_{2}$ & & 0.496 & 0.746 \\
\hline$a_{86}$ & 0.858 & $0.502 \quad C_{1}$ & & 0.785 & 0.773 \\
\hline$a_{87}$ & 0.51 & $0.194 \quad C_{1}$ & & 0.197 & 0.25 \\
\hline$a_{88}$ & 0.688 & 0.5 & $a_{188}$ & 0.466 & 0.245 \\
\hline$a_{89}$ & 0.718 & $0.98 \quad C_{3}$ & $a_{189}$ & 0.471 & 0.012 \\
\hline$a_{90}$ & 0.681 & $0.934 \quad C_{2}$ & $a_{190}$ & 0.394 & 0.915 \\
\hline$a_{91}$ & 0.492 & 0.971 & $a_{191}$ & 0.016 & 0.866 \\
\hline$a_{92}$ & 0.211 & $0.361 C_{1}$ & $a_{192}$ & 0.559 & 0.094 \\
\hline$a_{93}$ & 0.725 & $9 C_{1}$ & $a_{193}$ & 0.791 & 0.242 \\
\hline$a_{94}$ & 0.78 & $y$ & $a_{194}$ & 0.48 & 0.736 \\
\hline$a_{95}$ & & $C_{2}$ & $a_{195}$ & 0.332 & 0.13 \\
\hline$a_{96}$ & & $C_{1}$ & $a_{196}$ & 0.861 & 0.836 \\
\hline$a_{97}$ & & $0.734 \quad C_{2}$ & $a_{197}$ & 0.11 & 0.022 \\
\hline$a_{98}$ & & $0.589 \quad C_{1}$ & $a_{198}$ & 0.116 & 0.552 \\
\hline$a_{99}$ & 0.119 & $0.784 \quad C_{1}$ & $a_{199}$ & 0.617 & 0.969 \\
\hline$a_{100}$ & 0.891 & $0.392 C_{1}$ & $a_{200}$ & 0.934 & 0.725 \\
\hline
\end{tabular}

Appendix B. Classification results of the proposed approach and UTADIS. 


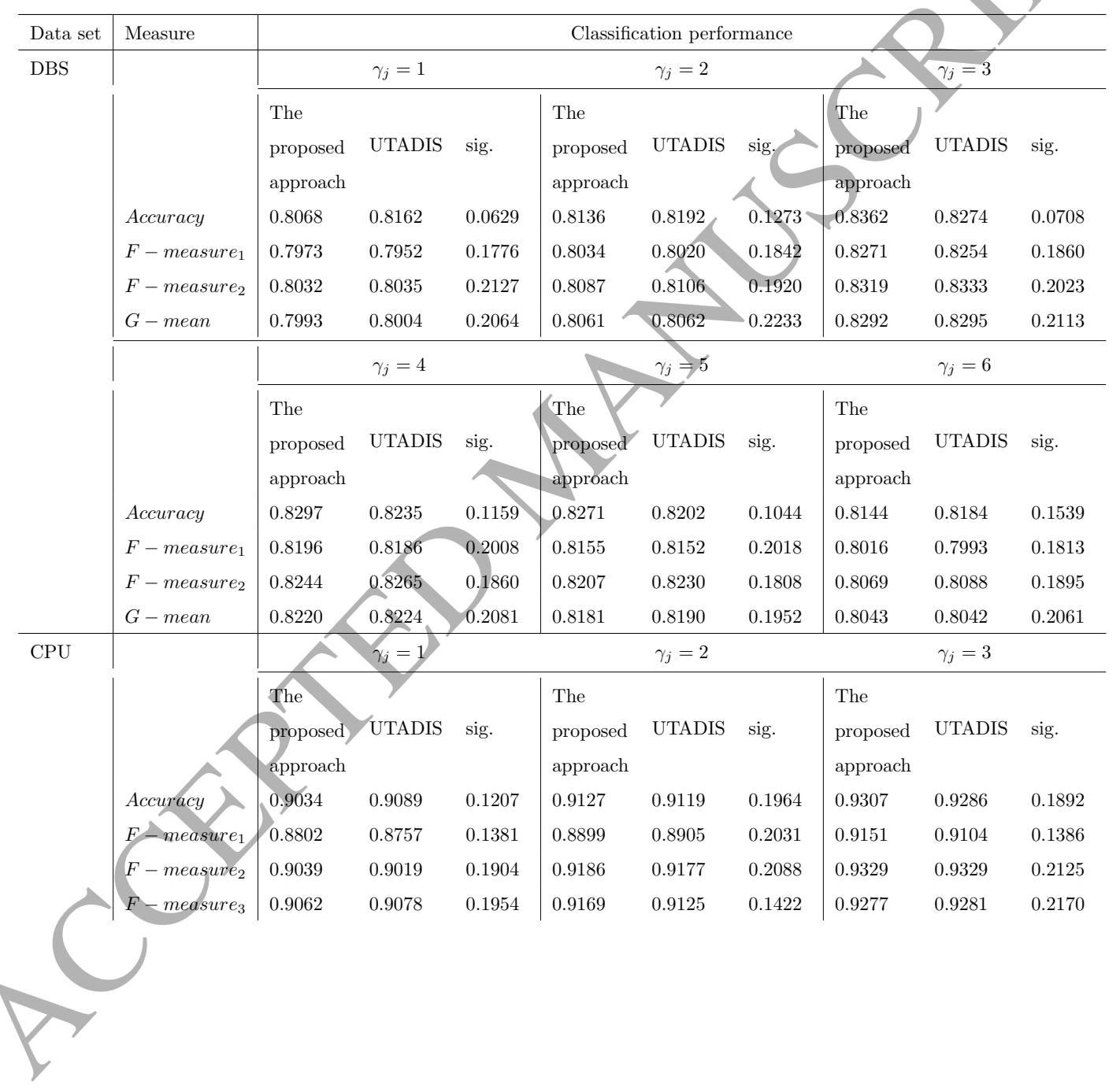




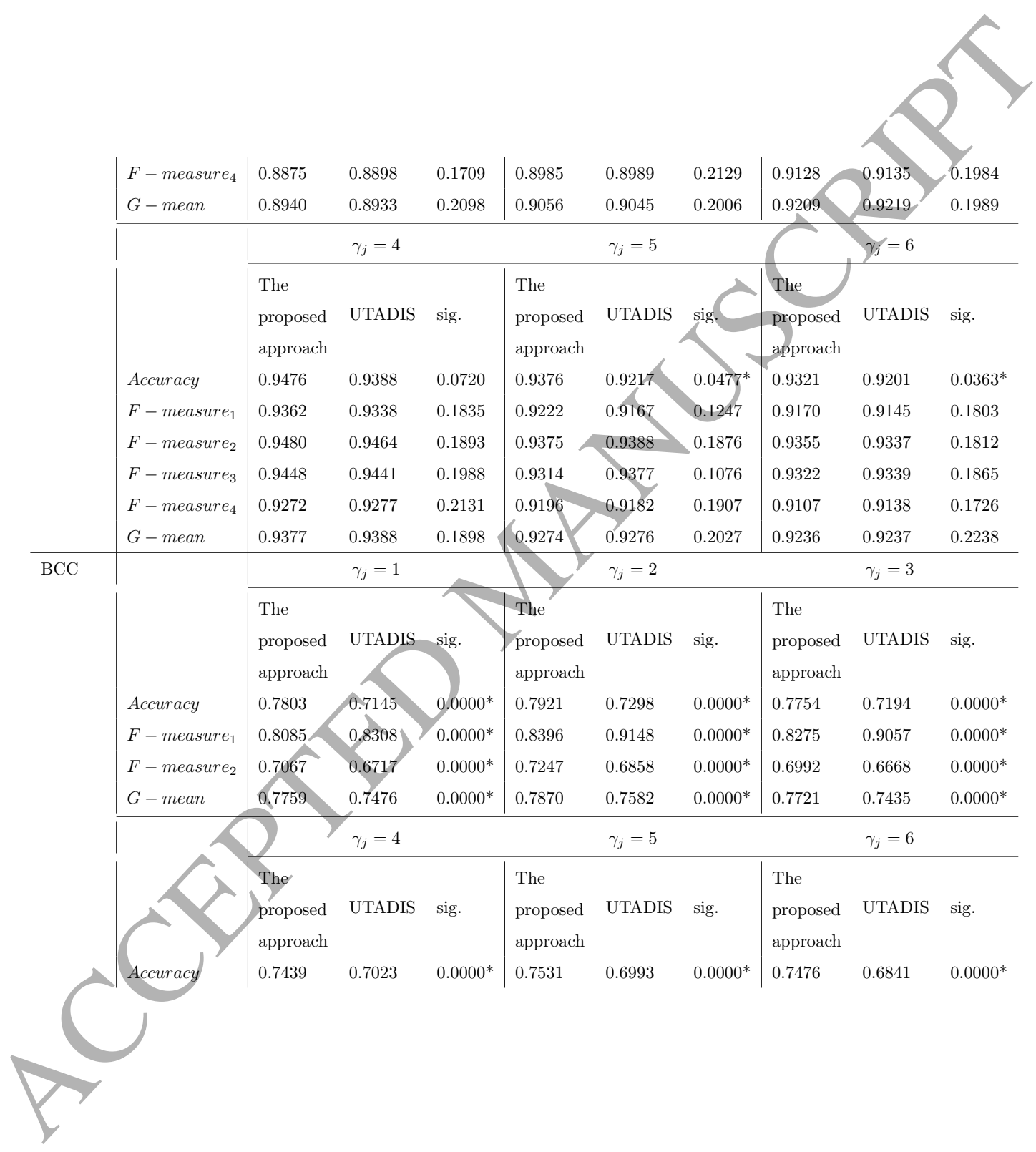




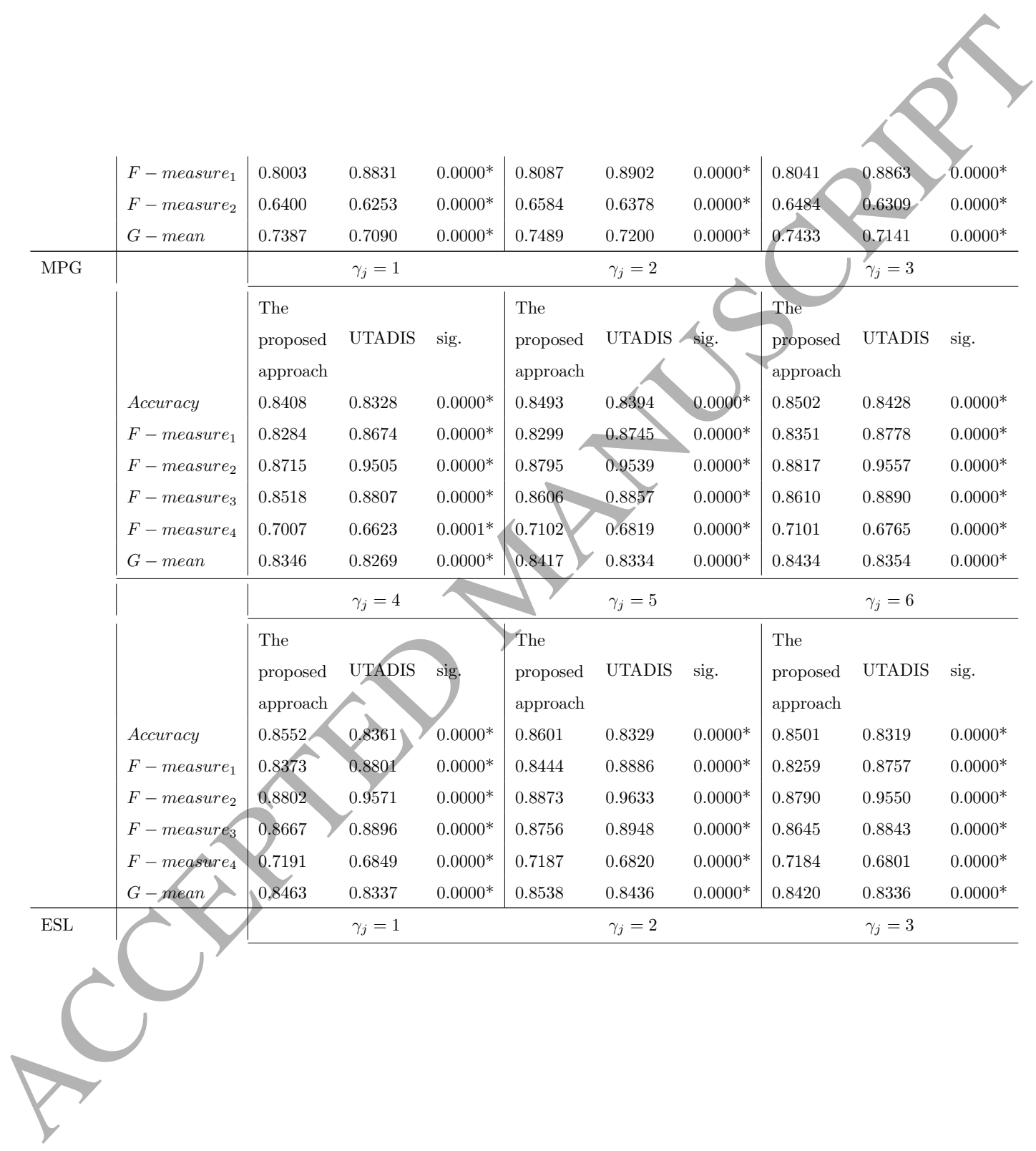




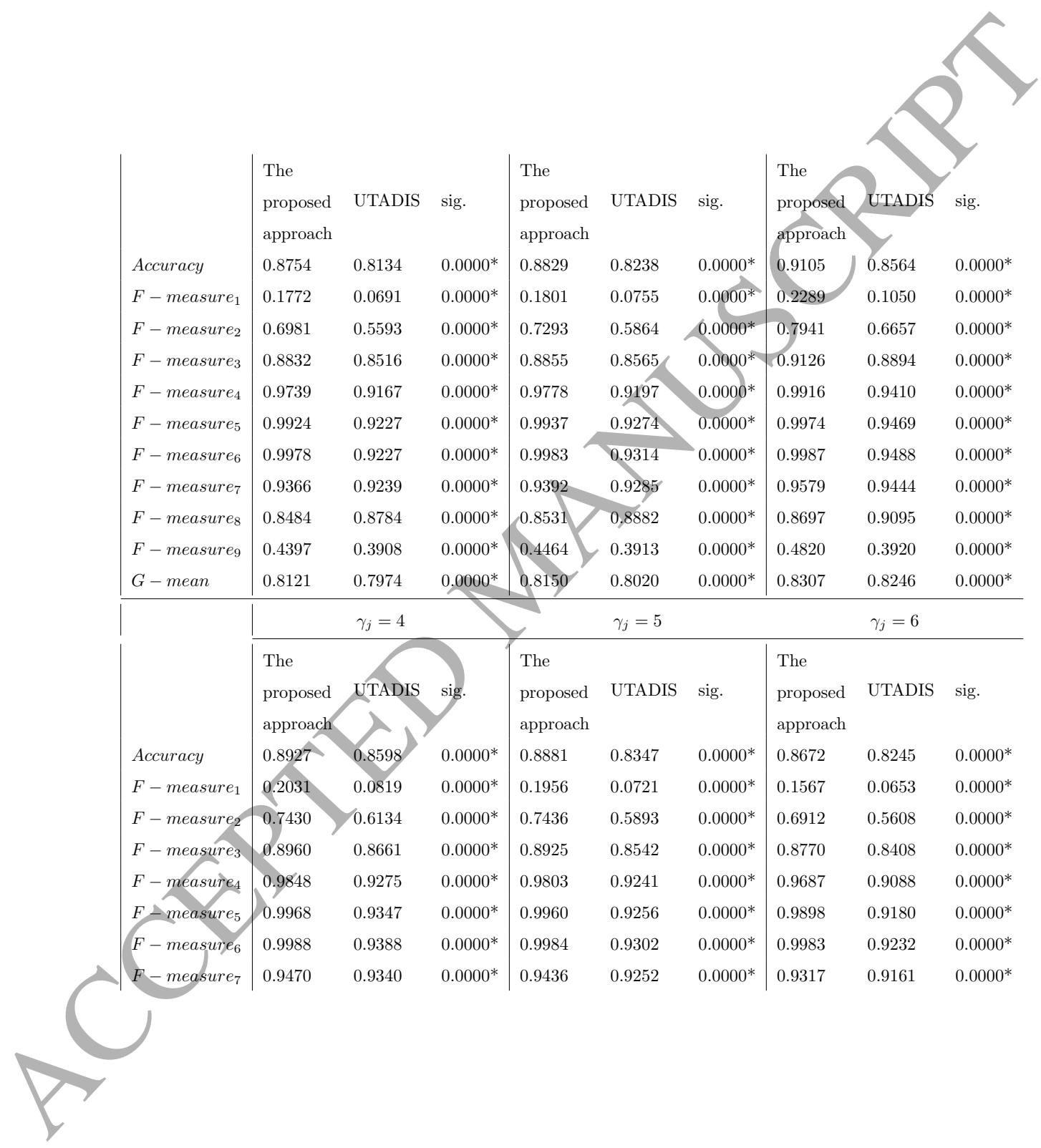




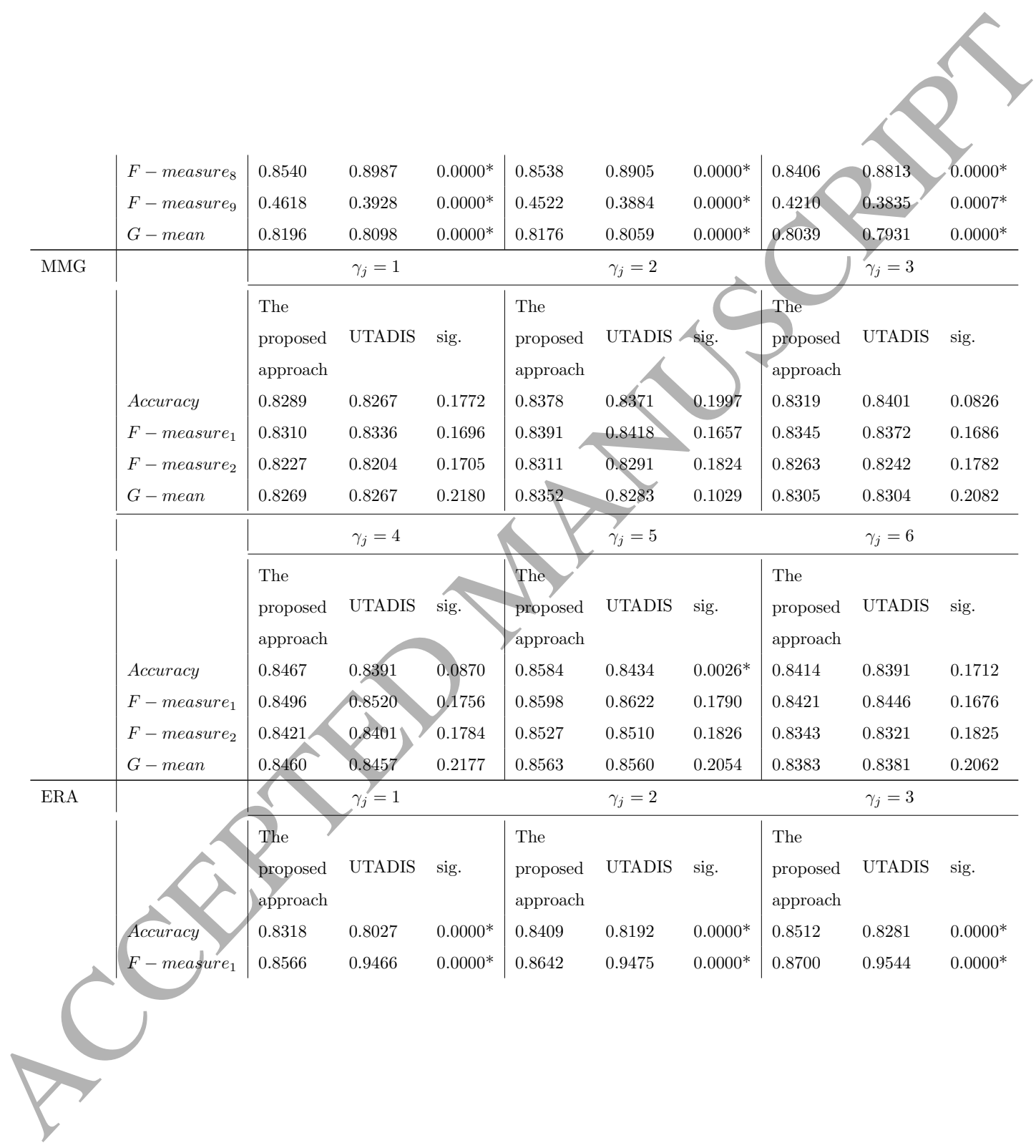




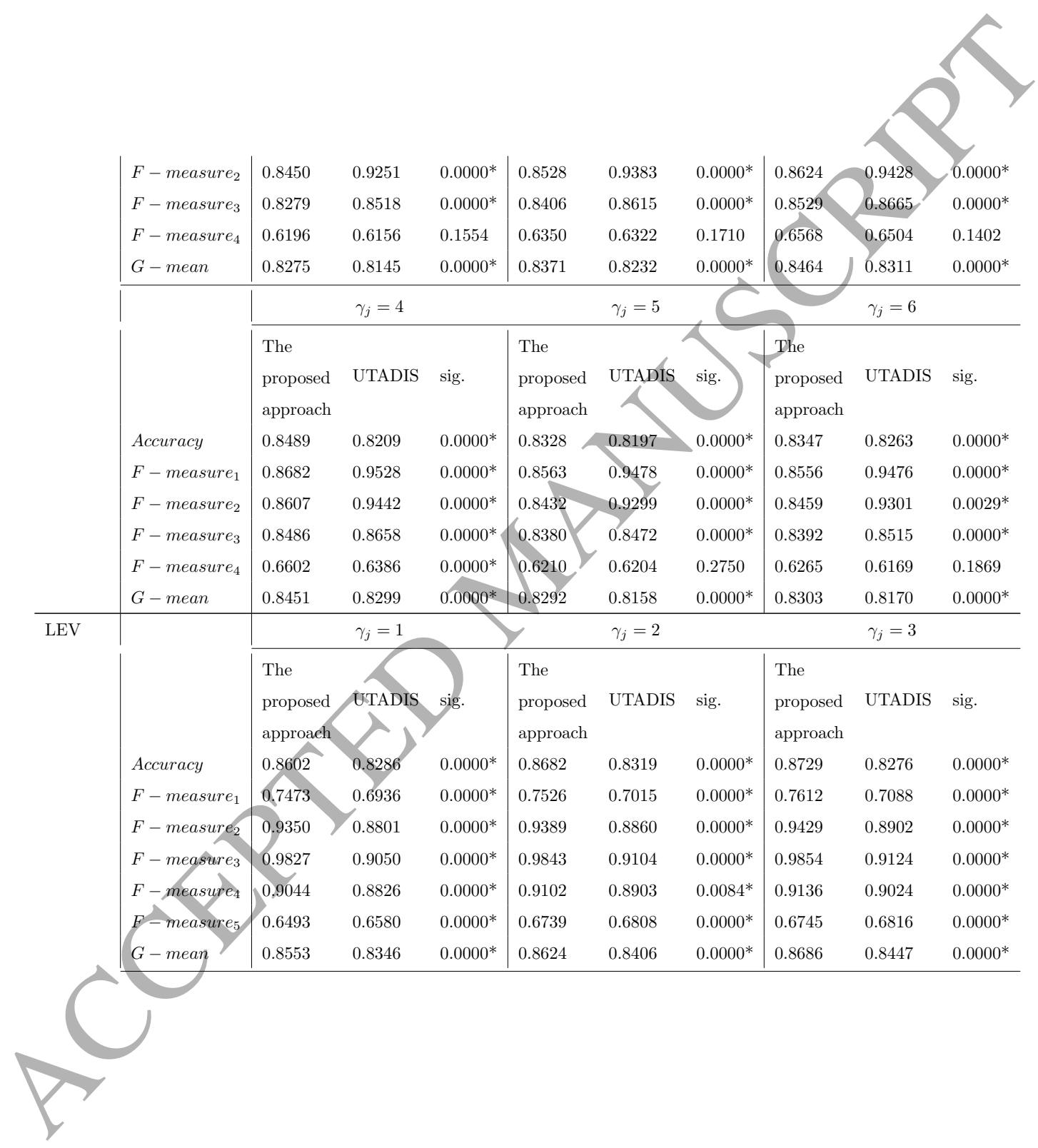









\begin{tabular}{|c|c|c|c|c|c|c|c|c|c|}
\hline Accuracy & 0.9328 & 0.8911 & $0.0000^{*}$ & 0.9431 & 0.8834 & $0.0000^{*}$ & 02 & & $0000^{*}$ \\
\hline$F-$ measure $_{1}$ & 0.9933 & 0.9593 & $0.0000^{*}$ & 0.9939 & 0.9642 & $0.0000^{*}$ & & & $0.0000^{*}$ \\
\hline$F-$ measure $_{2}$ & 0.9888 & 0.9167 & $0.0000^{*}$ & 0.9900 & 0.9245 & $0.0000^{*}$ & & 7 & $0.0000^{*}$ \\
\hline$F-$ measure $_{3}$ & 0.8251 & 0.8082 & $0.0000^{*}$ & 0.8233 & 0.8221 & 0.1899 & .8171 & 0.8042 & $0.0012^{*}$ \\
\hline$F-$ measure $_{4}$ & 0.8175 & 0.7773 & $0.0010^{*}$ & 0.8175 & 0.8023 & $0.0000^{*}$ & & 0.7822 & $0.0004^{*}$ \\
\hline$G-$ mean & 0.9300 & 0.8625 & $0.0000^{*}$ & 0.9373 & 0.8666 & $0.0000^{*}$ & 0.9246 & 0.8590 & $0.0000^{*}$ \\
\hline
\end{tabular}


Appendix C. Classification results of the proposed approach without and with the balancing algorithm.

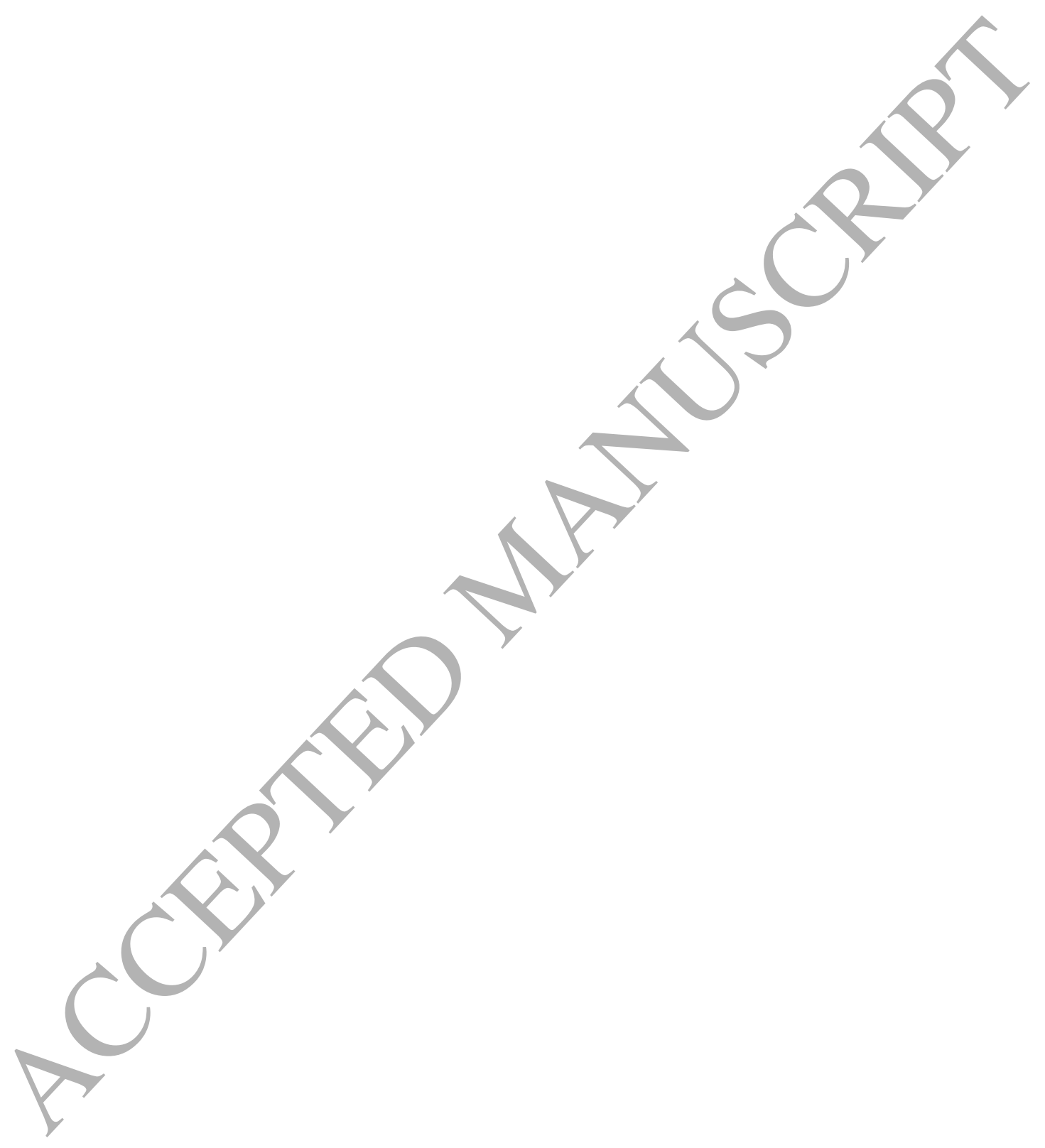




\begin{tabular}{|c|c|c|c|c|c|c|c|c|c|c|}
\hline Data set & Measure & \multicolumn{9}{|c|}{ Classification performance } \\
\hline \multirow[t]{12}{*}{ DBS } & & \multicolumn{3}{|c|}{$\gamma_{j}=1$} & \multicolumn{3}{|c|}{$\gamma_{j}=2$} & & \multirow[b]{2}{*}{$\begin{array}{l}\text { With } \\
\text { balancing }\end{array}$} & \multirow[b]{2}{*}{ sig. } \\
\hline & & $\begin{array}{l}\text { Without } \\
\text { balancing }\end{array}$ & $\begin{array}{l}\text { With } \\
\text { balancing }\end{array}$ & sig. & $\begin{array}{l}\text { Without } \\
\text { balancing }\end{array}$ & $\begin{array}{l}\text { With } \\
\text { balancing }\end{array}$ & 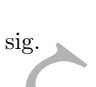 & $\begin{array}{l}\text { Without } \\
\text { balancing }\end{array}$ & & \\
\hline & Accuracy & 0.7265 & 0.8068 & $0.0000^{*}$ & 0.7995 & 0.8136 & $0.0000^{*}$ & 0.8247 & 0.8362 & $0.0000^{*}$ \\
\hline & $F-$ measure $_{1}$ & 0.7329 & 0.7973 & $0.0000^{*}$ & 0.7899 & 0.80 & $000^{*}$ & 0.7889 & 0.8271 & $0.0000^{*}$ \\
\hline & $F-$ measure $_{2}$ & 0.7786 & 0.8032 & $0.0000^{*}$ & 0.7378 & & $0.0000^{*}$ & 0.7507 & 0.8319 & $0.0000^{*}$ \\
\hline & $G-$ mean & 0.7695 & 0.7993 & $0.0000^{*}$ & 0.7672 & 0.80 & $0.0000^{*}$ & 0.8200 & 0.8292 & $0.0000^{*}$ \\
\hline & & \multicolumn{3}{|c|}{$\gamma_{j}=4$} & \multicolumn{3}{|c|}{$\gamma_{j}=5$} & \multicolumn{3}{|c|}{$\gamma_{j}=6$} \\
\hline & & $\begin{array}{l}\text { Without } \\
\text { balancing }\end{array}$ & $\begin{array}{l}\text { With } \\
\text { balancing }\end{array}$ & sig. & Withou & balancing & sig. & $\begin{array}{l}\text { Without } \\
\text { balancing }\end{array}$ & $\begin{array}{l}\text { With } \\
\text { balancing }\end{array}$ & sig. \\
\hline & Accuracy & 0.7773 & 0.8297 & $0.0000^{*}$ & 1 & 0.8271 & $0.0000^{*}$ & 0.8048 & 0.8144 & $0.0000^{*}$ \\
\hline & $F-$ measure $_{1}$ & 0.7415 & 0.8196 & $0.0000^{*}$ & 0.7796 & 0.8155 & $0.0000^{*}$ & 0.7558 & 0.8016 & $0.0000^{*}$ \\
\hline & $F-$ measure $_{2}$ & 0.8045 & 0.8244 & $0.0000^{*}$ & 0.7725 & 0.8207 & $0.0000^{*}$ & 0.7974 & 0.8069 & $0.0000^{*}$ \\
\hline & $G-$ mean & 0.7874 & 0.8220 & $0.0000^{*}$ & 0.7499 & 0.8181 & $0.0000^{*}$ & 0.7483 & 0.8043 & $0.0000^{*}$ \\
\hline \multirow[t]{9}{*}{ CPU } & & & & & \multicolumn{3}{|c|}{$\gamma_{j}=2$} & \multicolumn{3}{|c|}{$\gamma_{j}=3$} \\
\hline & & & balans & & $\begin{array}{l}\text { Without } \\
\text { balancing }\end{array}$ & $\begin{array}{l}\text { With } \\
\text { balancing }\end{array}$ & sig. & $\begin{array}{l}\text { Without } \\
\text { balancing }\end{array}$ & $\begin{array}{l}\text { With } \\
\text { balancing }\end{array}$ & sig. \\
\hline & Accuracy & 0.8812 & 0.9034 & $0.0000^{*}$ & 0.8436 & 0.9127 & $0.0000^{*}$ & 0.8377 & 0.9307 & $0.0000^{*}$ \\
\hline & & 3619 & 0.8802 & $0.0000^{*}$ & 0.8682 & 0.8899 & $0.0000^{*}$ & 0.8198 & 0.9151 & $0.0000^{*}$ \\
\hline & $F$ & 0.8619 & 0.9039 & $0.0000^{*}$ & 0.8723 & 0.9186 & $0.0000^{*}$ & 0.8694 & 0.9329 & $0.0000^{*}$ \\
\hline &  & 0.8485 & 0.9062 & $0.0000^{*}$ & 0.8839 & 0.9169 & $0.0000^{*}$ & 0.8379 & 0.9277 & $0.0000^{*}$ \\
\hline & & 0.8014 & 0.8875 & $0.0000^{*}$ & 0.8673 & 0.8985 & $0.0000^{*}$ & 0.8446 & 0.9128 & $0.0000^{*}$ \\
\hline & $G-$ mean & 0.8583 & 0.8940 & $0.0000^{*}$ & 0.8817 & 0.9056 & $0.0000^{*}$ & 0.8587 & 0.9209 & $0.0000^{*}$ \\
\hline & & \multicolumn{3}{|c|}{$\gamma_{j}=4$} & \multicolumn{3}{|c|}{$\gamma_{j}=5$} & \multicolumn{3}{|c|}{$\gamma_{j}=6$} \\
\hline
\end{tabular}




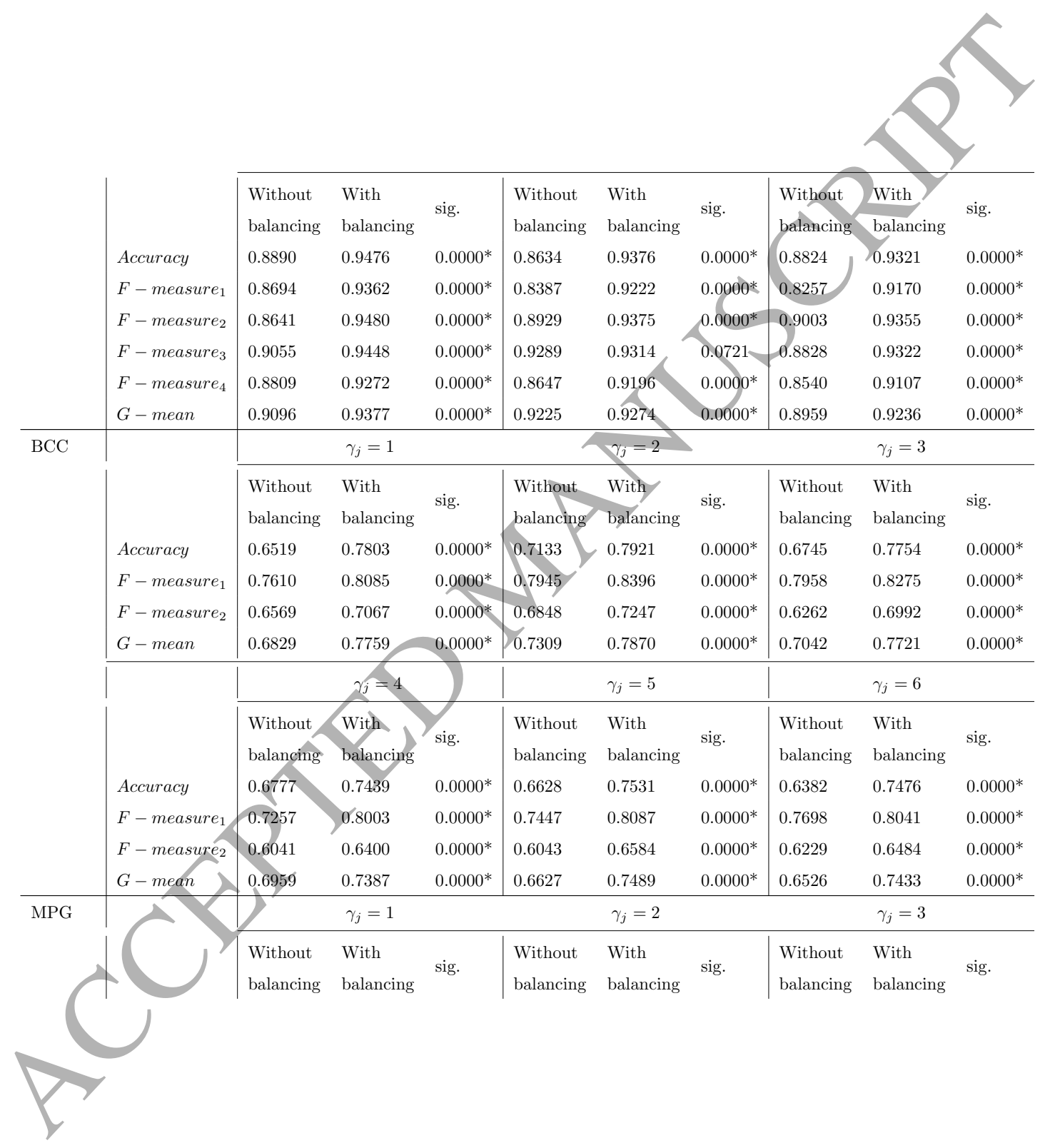




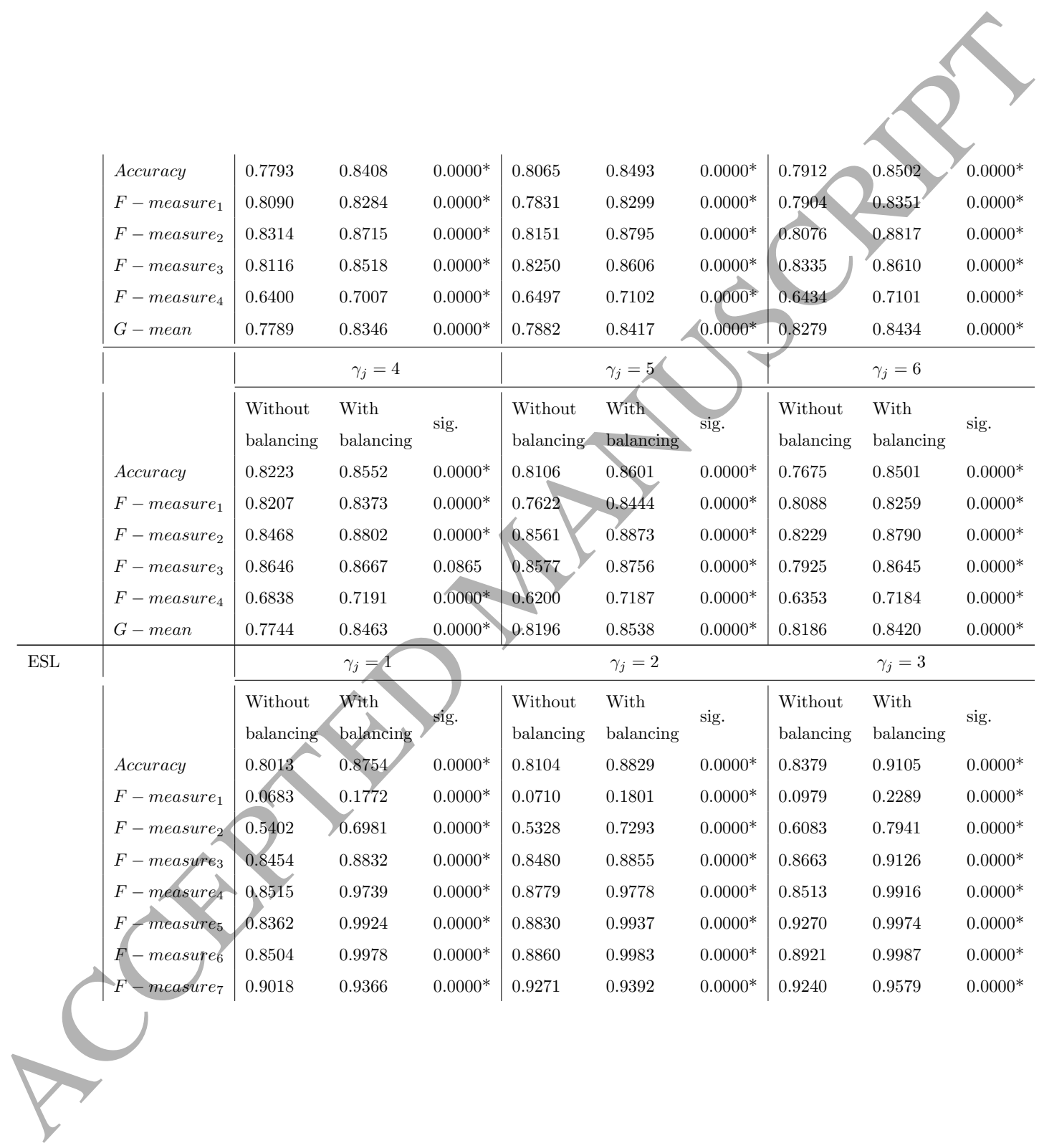




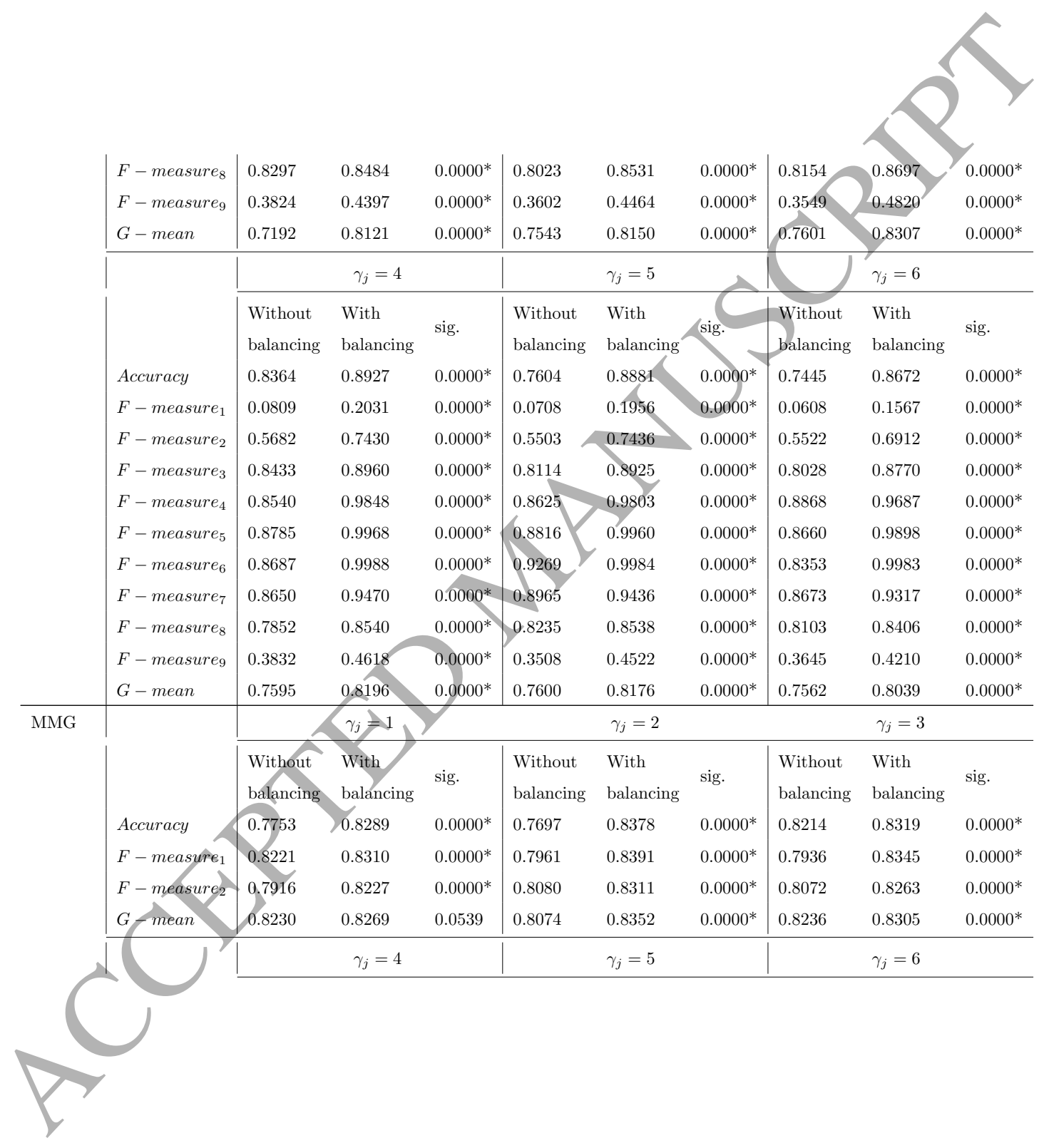




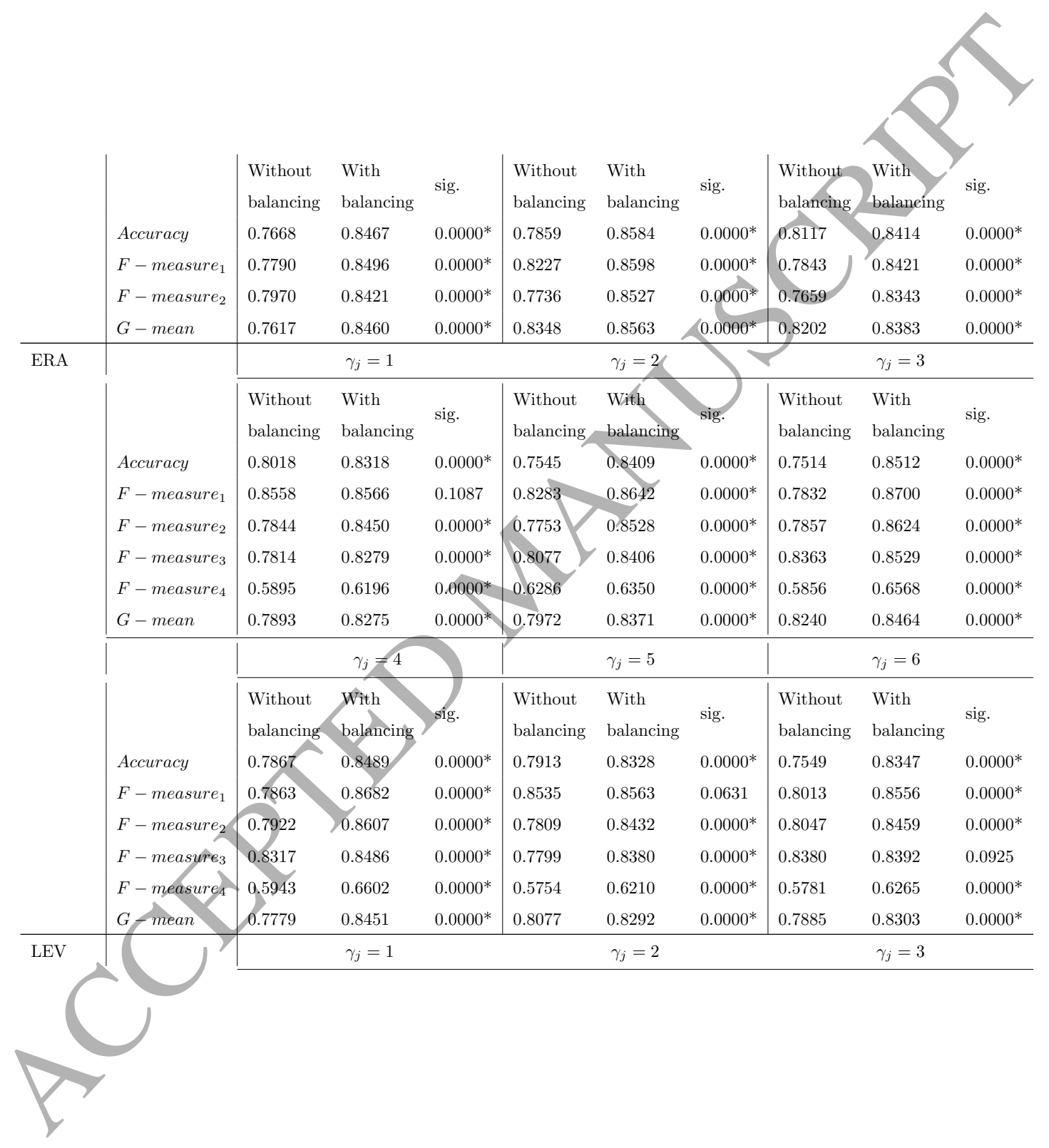




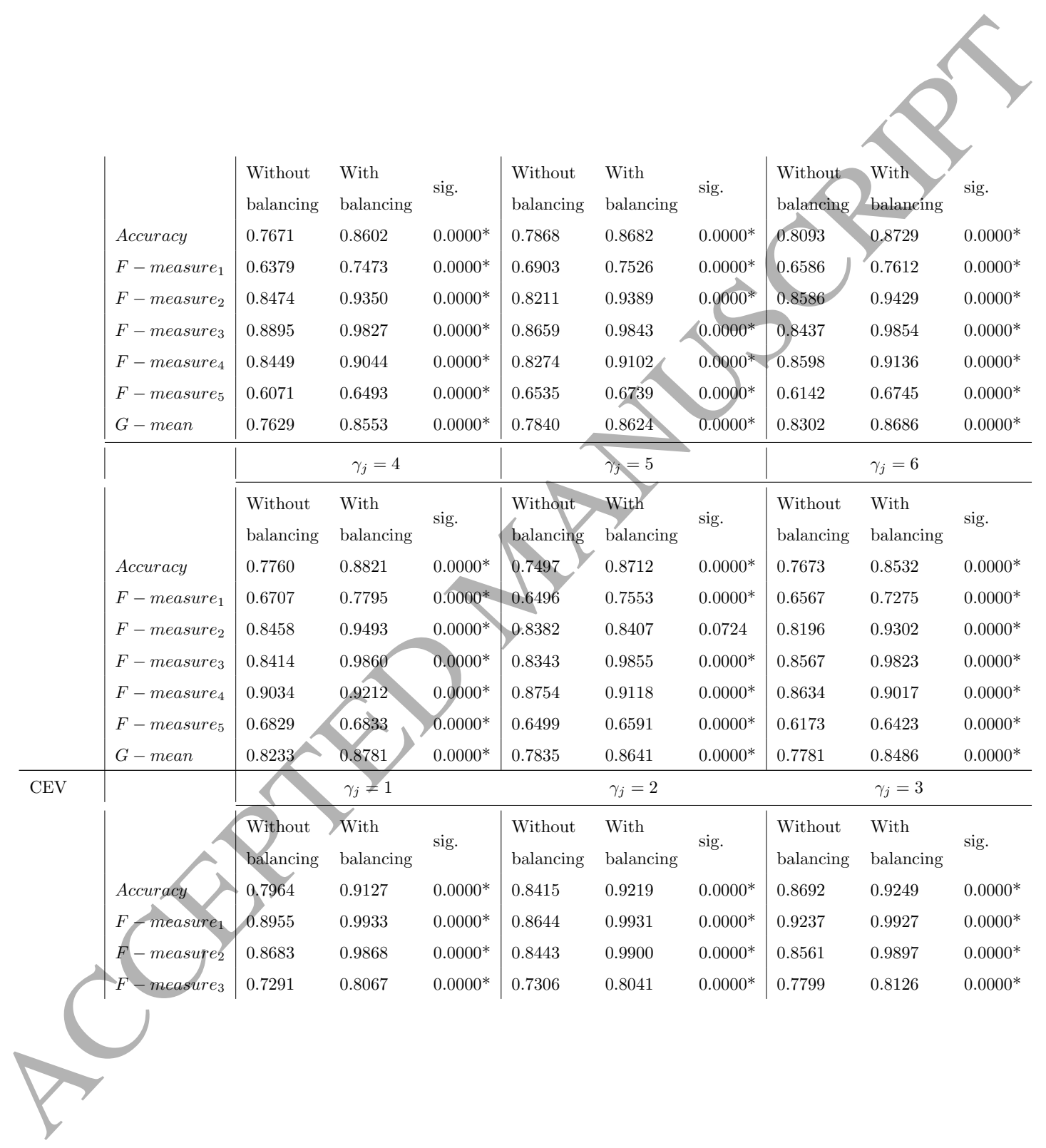




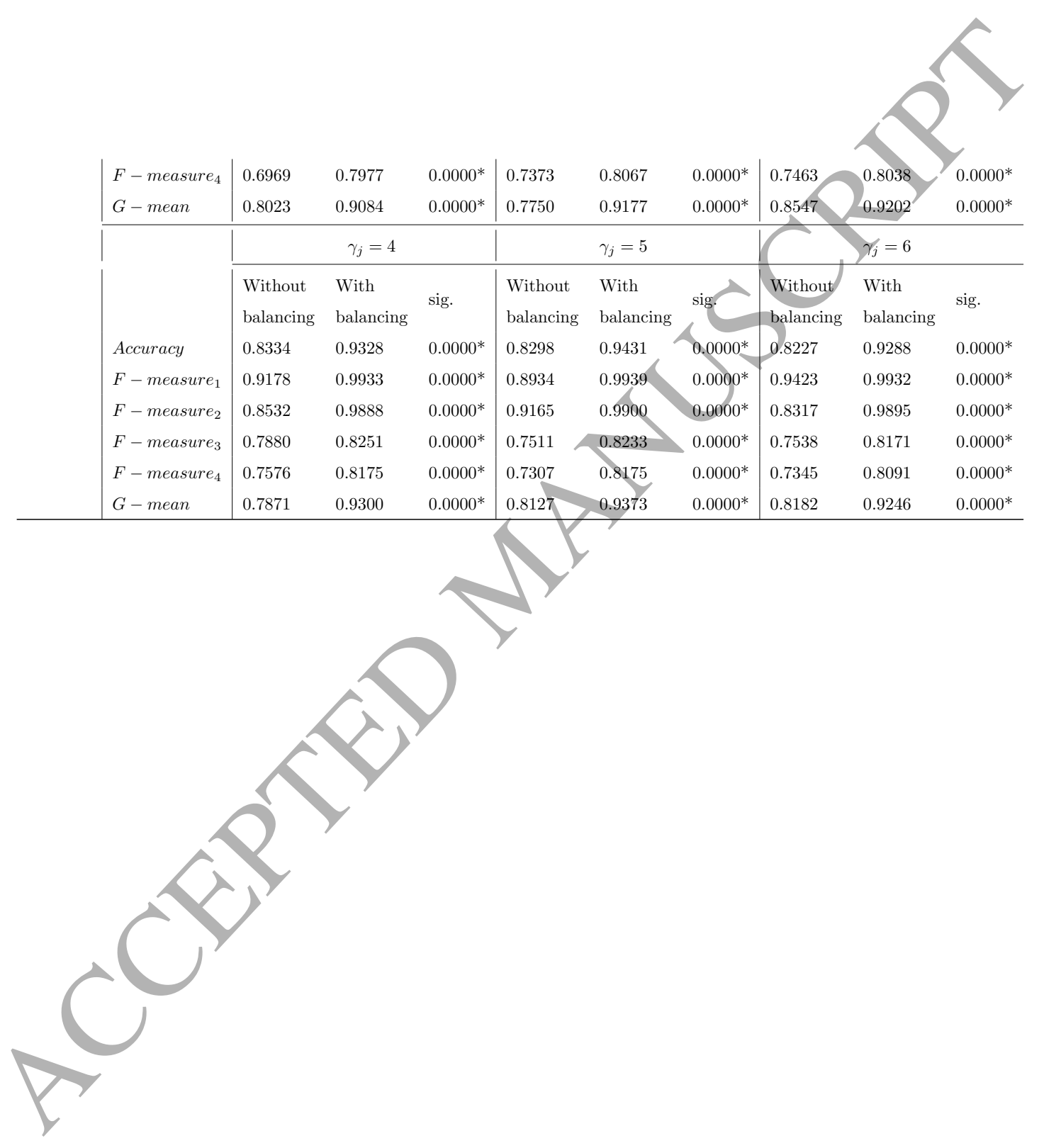

